\title{
Modeling and Analysis of Multiple Engine Aircraft Configurations for Fault Tolerant Control
}

Frederick M. Beamer

West Virginia University

Follow this and additional works at: https://researchrepository.wvu.edu/etd

\section{Recommended Citation}

Beamer, Frederick M., "Modeling and Analysis of Multiple Engine Aircraft Configurations for Fault Tolerant Control" (2011). Graduate Theses, Dissertations, and Problem Reports. 2295.

https://researchrepository.wvu.edu/etd/2295

This Thesis is protected by copyright and/or related rights. It has been brought to you by the The Research Repository @ WVU with permission from the rights-holder(s). You are free to use this Thesis in any way that is permitted by the copyright and related rights legislation that applies to your use. For other uses you must obtain permission from the rights-holder(s) directly, unless additional rights are indicated by a Creative Commons license in the record and/ or on the work itself. This Thesis has been accepted for inclusion in WVU Graduate Theses, Dissertations, and Problem Reports collection by an authorized administrator of The Research Repository @ WVU. For more information, please contact researchrepository@mail.wvu.edu. 


\title{
Modeling and Analysis of Multiple Engine Aircraft Configurations for Fault Tolerant Control
}

\author{
Frederick M. Beamer
}

Thesis submitted to the

College of Engineering and Mineral Resources

at West Virginia University

\author{
in partial fulfillment of the requirements \\ for the degree of
}

Master of Science

in

Aerospace Engineering

Committee:

Dr. Mario Perhinschi, Chair

Dr. John Loth

Dr. Jacky Prucz

Department of Mechanical and Aerospace Engineering

Morgantown, WV 


\section{Abstract \\ Modeling and Analysis of Multiple Engine Aircraft Configurations for Fault Tolerant Control}

\section{Frederick M. Beamer}

A formal framework is presented that allows for the analysis of the potential for using engine thrust control for aircraft actuator failure accommodation. Three sets of parameters have been identified as critical: number of engines and their position, engine thrust and throttle dynamics, and type and severity of the actuator failure. A mathematical model was developed that allows for the determination of the values of some of the parameters when the others are imposed such as determining the thrust control authority when the engine locations and Euler angles are known. Additionally, the engine locations can be determined when the thrust control authority and engine Euler angles are known and the engine Euler angles can be determined when the engine locations and thrust control authority are known. A MATLAB/Simulink simulation environment was built around a model of a large transport that can accommodate up to ten engines at different locations. A fuzzy logic controller was designed and employed for failure accommodation. The fuzzy logic controller utilizes the pilot lateral, longitudinal, and directional commands as well as the aircraft's pitch attitude, roll attitude, yaw attitude and respective angular rates as the inputs to the system and provides throttle commands for each engine based on its location with respect to the aircraft's center of mass. Failures of varying severity on the rudder, left or right aileron, and left or right elevator were implemented. The controller was capable of accommodating an extremely severe aileron failure and moderately severe rudder failure without additional pilot input. The controller was capable of mitigating some of the pilot command required for a moderate elevator failure. The simulation environment was used to verify the analytical results and to demonstrate the fault tolerant capabilities of multiple engine configurations. It proved to be a flexible and efficient tool for analysis and control system development. 


\section{Acknowledgements}

I would like to extend a very heartfelt thank you to my committee chairman, Dr. Mario Perhinschi, who gave me the opportunity, guidance, and support to complete this research and thesis. I would also like to thank my committee members, Dr. Jacky Prucz and Dr. John Loth, for agreeing to help me reach this point in my academic career. I would also like to thank Matthew Cunningham, Ondrej Karas, and Phil Evans for being great sounding boards and helping me out in troubleshooting some of the random quirks of MATLAB and Simulink. I would like to thank my friends that I have met in Morgantown as well as my "sister" and "brother" from home. I want to thank them for putting up with me during the stressful times and for always offering their encouragement to see this through the end. I also want to thank my grandparents and great aunt and uncle as well as the rest of my extended family and friends for keeping me grounded. Finally, I would like to thank my parents, Terry and Rebecca Beamer, for always being there for me through thick and thin offering their unending love and support and for encouraging me to follow my dreams. 


\section{Table of Contents}

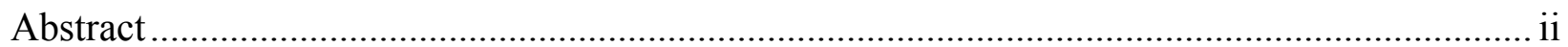

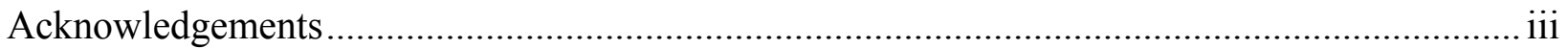

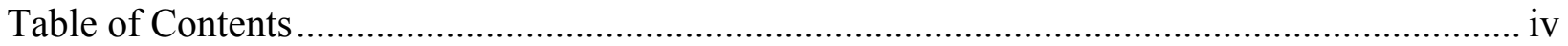

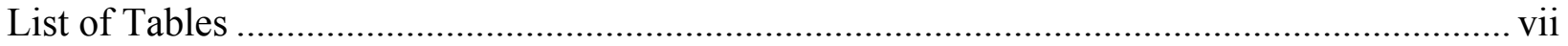

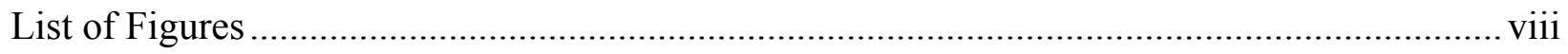

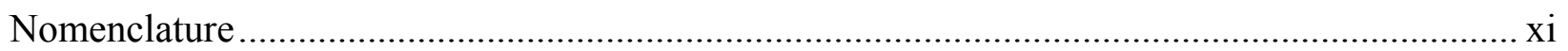

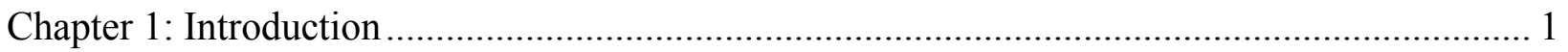

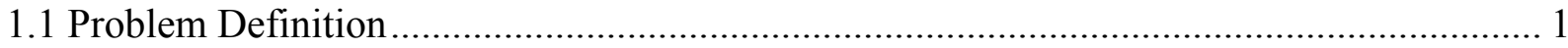

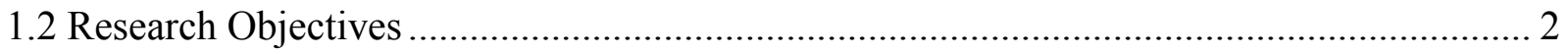

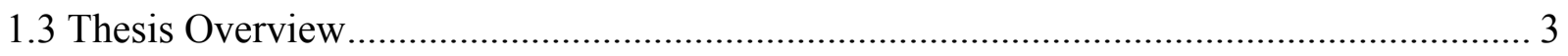

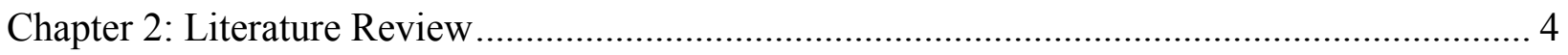

2.1 Modeling and Simulation of Actuator Failures............................................................... 4

2.2 Engine Use in Actuator Failure Accommodation ........................................................... 5

Chapter 3: Multiple Engine Modeling for Fault Tolerant Control Purposes .................................. 8

3.1 Reference Frames and Systems of Coordinates .............................................................. 8

3.2 Vector Notations and Transformations ………………................................................ 10

3.3 Engine Force and Moment Compensation ....................................................................... 11

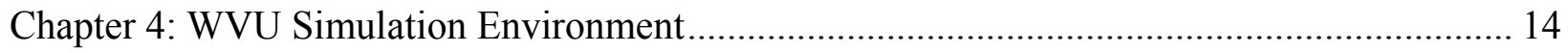

4.1 General Description of the WVU Simulation Environment ………................................... 14 


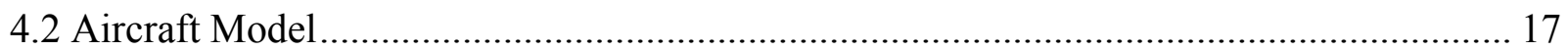

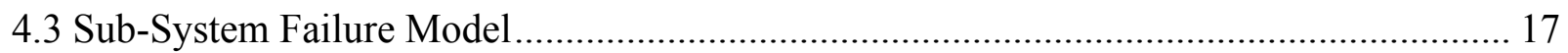

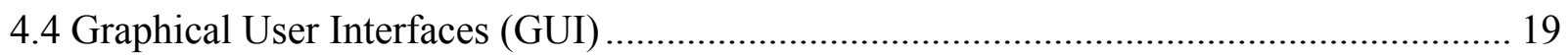

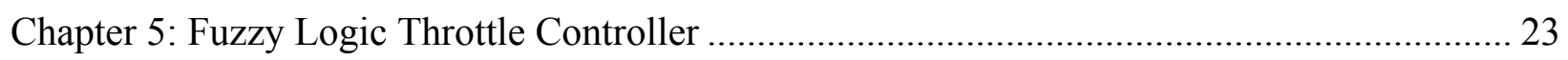

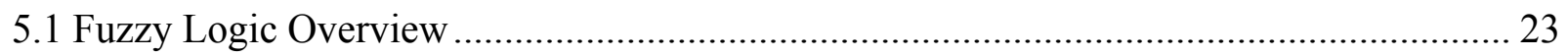

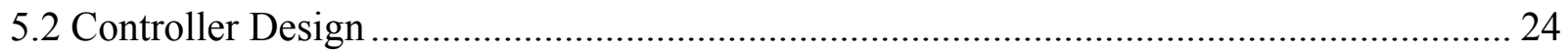

5.3 Implementing the Fuzzy Logic Controller ................................................................... 30

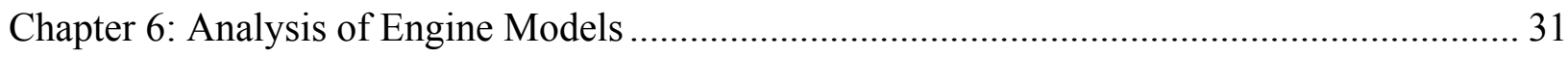

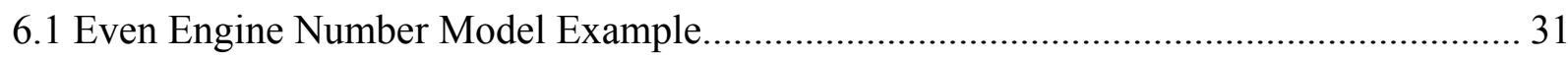

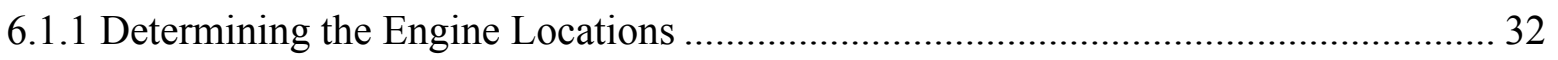

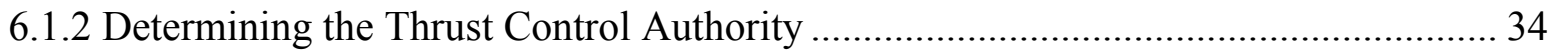

6.1.3 Determining the Engine Euler Angles ...................................................................... 35

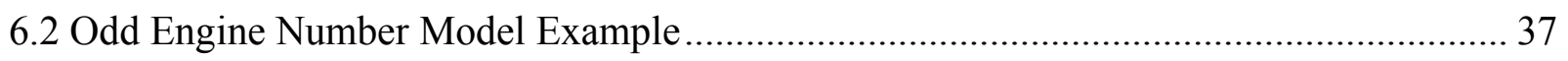

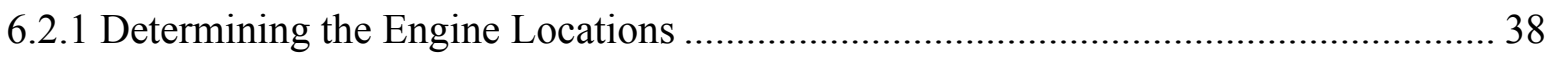

6.2.2 Determining the Thrust Control Authority ................................................................ 42

6.2.3 Determining the Engine Euler Angles .................................................................... 43

Chapter 7: Analysis of Engine Compensation for Specific Actuator Failures .............................. 46

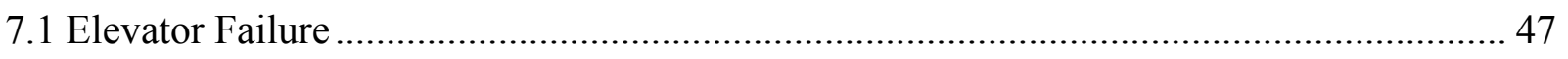

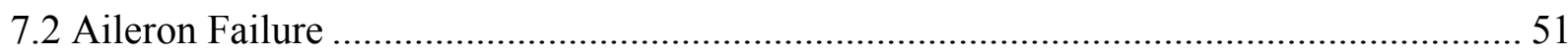

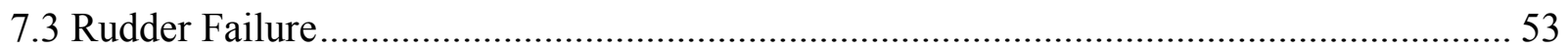


Chapter 8: Simulation Results and Discussion

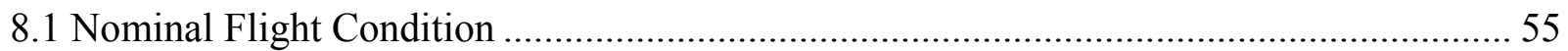

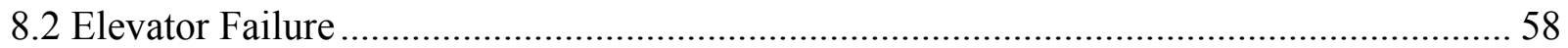

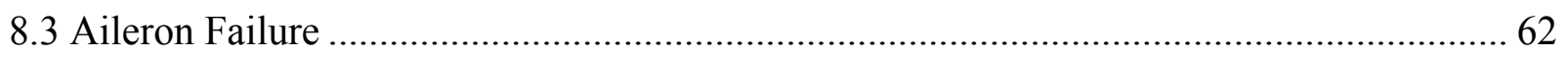

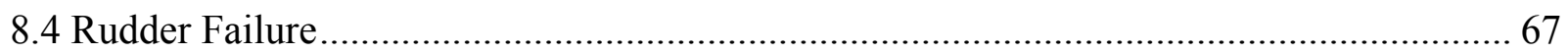

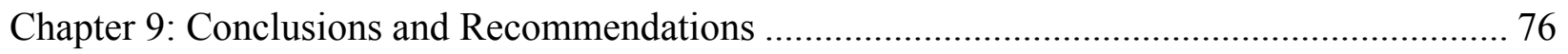

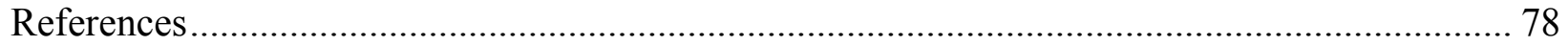

Appendix A: Analysis of Engine Compensation for a Tri-Engine Aircraft Configuration......... A-1

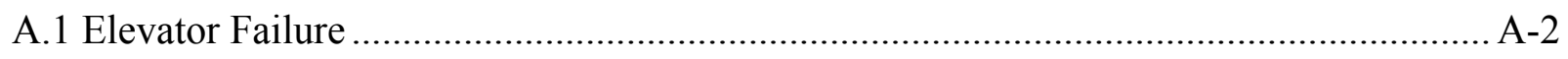

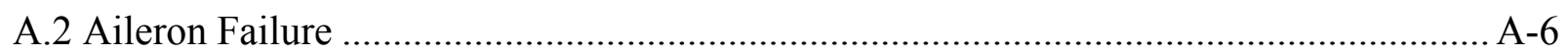

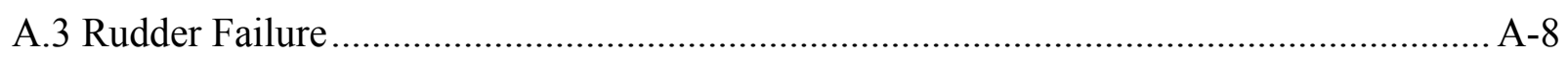

Appendix B: Simulation Environment User Manual............................................................ B-1 


\section{List of Tables}

Table 5-1: Output Inference Rules Matrix....................................................................... 29

Table 6-1: Conditions for a Non-Zero Determinant for Equation (6.16) ............................... 34

Table 6-2: Conditions for a Non-Zero determinate for Equation (6.50)............................... 41

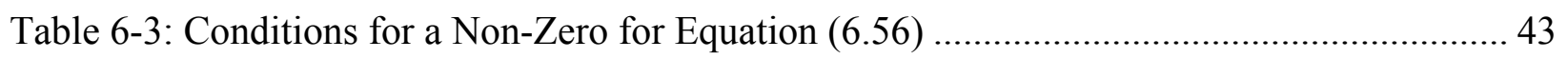




\section{List of Figures}

Figure 3-1: Aircraft Tri-View with the Body Reference Frame .......................................... 9

Figure 4-1: General Architecture of the WVU Simulation Environment................................ 14

Figure 4-2: Simulink Model of the WVU Simulation Environment ...................................... 15

Figure 4-3: WVU Simulation Environment Interface ................................................... 16

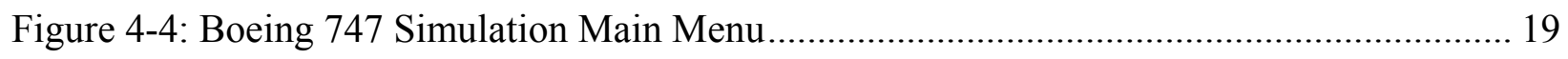

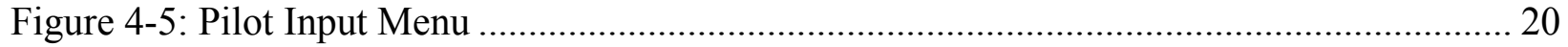

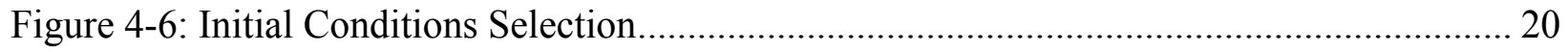

Figure 4-7: Control Surface Failure Conditions Menu ......................................................... 21

Figure 4-8: Engine Selection and Position Menu ................................................................ 22

Figure 4-9: Flight Visualization Menu .............................................................................. 22

Figure 5-1: Simulink Fuzzy Logic Model Overview......................................................... 24

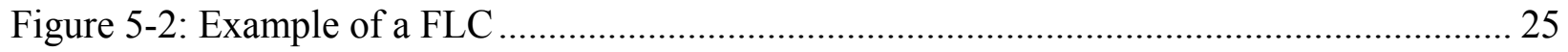

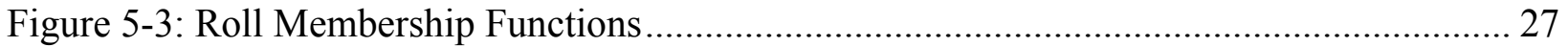

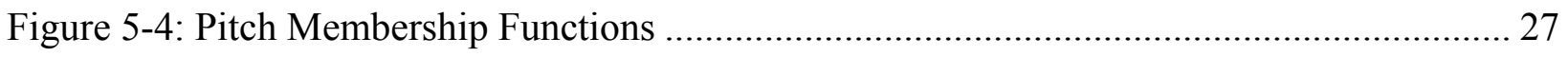

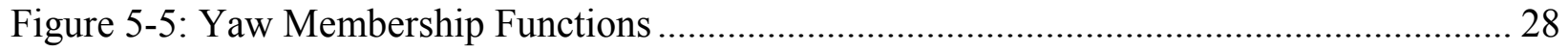

Figure 5-6: Output Membership Function for Roll, Pitch, and Yaw Channels .......................... 28

Figure 8-1: Control Surface Deflections for the Elevators, Ailerons, and Rudder for the Nominal

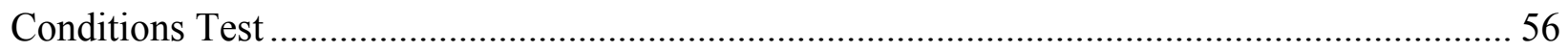

Figure 8-2: Roll, Pitch, and Yaw Rates for the Nominal Conditions Test .............................. 57

Figure 8-3: Pilot Throttle Command and the FLC Throttle Command to Each Engine for the

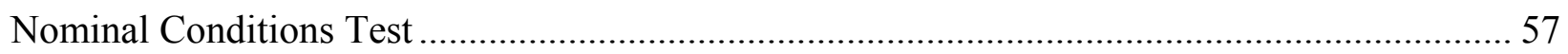

Figure 8-4: Control Surface Deflections for the -10 Degree Left Elevator Failure Case with the

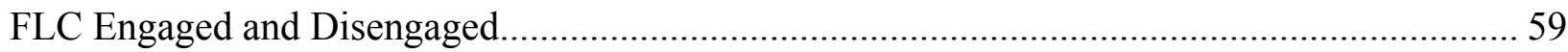

Figure 8-5: Throttle Commands for the -10 Degree Left Elevator Failure Case with the FLC

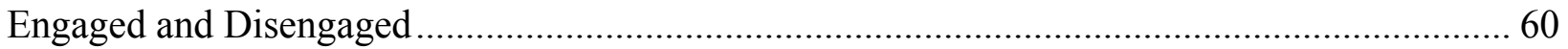

Figure 8-6: Roll, Pitch, and Yaw Rates for the -10 Degree Left Elevator Failure Case with the

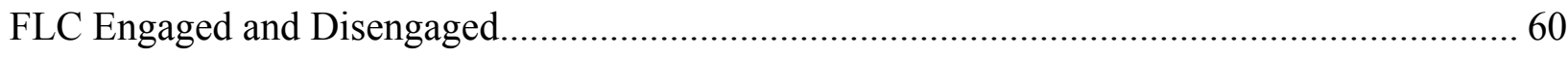

Figure 8-7: Control Surface Deflections for the +10 Degree Left Elevator Failure Case with the

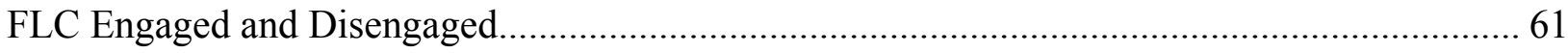


Figure 8-8: Throttle Commands for the +10 Degree Left Elevator Failure Case with the FLC Engaged and Disengaged

Figure 8-9: Roll, Pitch, and Yaw Rates for the +10 Degree Left Elevator Failure Case with the FLC Engaged and Disengaged.

Figure 8-10: Control Surface Deflections for the +10 Degree Left Aileron Failure Case with the FLC Engaged and Disengaged.

Figure 8-11: Throttle Commands for the +10 Degree Left Aileron Failure Case with the FLC Engaged and Disengaged 64

Figure 8-12: Roll, Pitch, and Yaw Rates for the +10 Degree Left Aileron Failure Case with the FLC Engaged and Disengaged.

Figure 8-13: Control Surface Deflections for the +20 Degree Left Aileron Failure Case with the FLC Engaged and Disengaged.

Figure 8-14: Throttle Commands for the +20 Degree Left Aileron Failure Case with the FLC Engaged and Disengaged 66

Figure 8-15: Roll, Pitch, and Yaw Rates for the +20 Degree Left Aileron Failure Case with the FLC Engaged and Disengaged.

Figure 8-16: Control Surface Deflections for the +10 Degree Rudder Failure Case with the FLC Engaged and Disengaged 69

Figure 8-17: Throttle Commands for the +10 Degree Rudder Failure Case with the FLC Engaged and Disengaged. 70

Figure 8-18: Roll, Pitch, and Yaw Rates for the +10 Degree Rudder Failure Case with the FLC Engaged and Disengaged

Figure 8-19: Yaw Rate without the Initial Peak for the +10 Degree Rudder Failure Case with the FLC Engaged and Disengaged 71

Figure 8-20: Control Surface Deflections for the +15 Degree Rudder Failure Case with the FLC

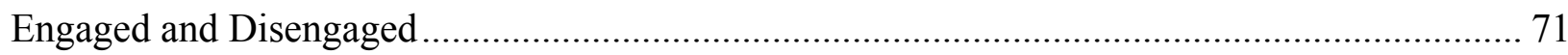

Figure 8-21: Throttle Commands for the +15 Degree Rudder Failure Case with the FLC Engaged and Disengaged..... 72

Figure 8-22: Roll, Pitch, and Yaw Rates for the +15 Degree Rudder Failure Case with the FLC Engaged and Disengaged 
Figure 8-23: Throttle Commands for the +15 Degree Rudder Failure when the Thrust Potential is Increased 73

Figure 8-24: Control Surface Deflections for the +15 Degree Rudder Failure when the Thrust

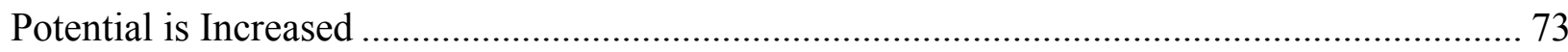

Figure 8-25: Roll, Pitch, and Yaw Rates for the +15 Degree Rudder Failure when the Thrust Potential is Increased

Figure 8-26: Throttle Commands for the +15 Degree Rudder Failure when the Outer Engine

Locations are Moved.

Figure 8-27: Control Surface Deflections for the +15 Degree Rudder Failure when the Outer

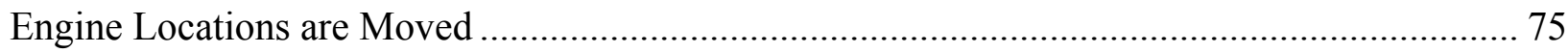

Figure 8-28: Roll, Pitch, and Yaw Rates for the +15 Degree Rudder Failure when the Outer

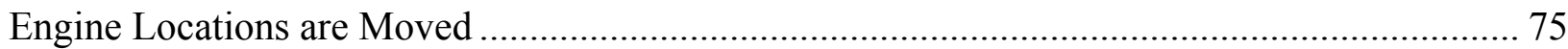

Figure B-0-1: MATLAB Command Window with the Current Directory Set........................ B-1

Figure B-0-2: Opening the WVU Boeing 747 Simulation Menu.......................................... B-2

Figure B-0-3: Boeing 747 Simulation Main Menu................................................................... B-2

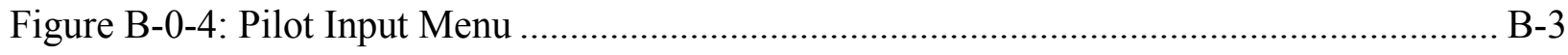

Figure B-0-5: Proper Loading of the Pilot Input Files......................................................... B-3

Figure B-0-6: Initial Conditions Selection........................................................................ B-4

Figure B-0-7: Control Surface Failure Conditions Menu ................................................... B-4

Figure B-0-8: Engine Selection and Position Menu ............................................................. B-5

Figure B-0-9: WVU Boeing 747 Simulink Model ............................................................... B-6

Figure B-0-10: Flight Visualization Menu ................................................................... B-7

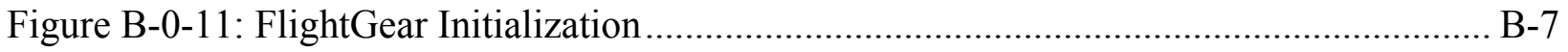

Figure B-0-12: FlightGear Window with Operating Flight Visualization.............................. B-8

Figure B-0-13: Simulink Start and Stop Simulation Button and Simulation Time Highlighted B-8 


\section{Nomenclature}

$\delta_{j C}=$ commanded deflection of control surface $\mathrm{j}$

$\delta_{j F} \quad=$ deflection of failed control surface $\mathrm{j}$

$F_{B} \quad=$ fixed body reference frame with respect to the aircraft

$F_{E} \quad=$ Earth reference frame

FLC $\quad=$ Fuzzy Logic Controller

$F_{M_{i}} \quad=$ fixed body reference frame with respect $t$ the ith engine

$\vec{M}_{e_{i}} \quad=$ moment produced by the ith engine

$\vec{M}_{C i} \quad=$ moment produced by one aerodynamic surface $\mathrm{i}$

$\vec{M}_{F j} \quad=$ moment produced by failed aerodynamic control surface $\mathrm{j}$

$N \quad=$ number of engines

$P_{i} \quad=$ point of application of thrust for the ith engine

$O \quad=$ center of mass of the aircraft

$O X Y Z=$ system of coordinates in relation to $F_{B}$ with the origin at the mass center of the vehicle

$O_{E} X_{E} Y_{E} Z_{E} \quad=$ system of coordinates in relation to $F_{B}$ with the origin at an arbitrary reference point on the surface of the earth 


$$
\begin{aligned}
& R F \quad=\text { reference frame } \\
& \vec{r}^{O P_{i}} \quad=\text { position vector of the point } \mathrm{Pi} \text { with respect to point } \mathrm{O} \\
& {\left[\vec{r}^{O P_{i}}\right]_{A} \quad=\text { components of position vector with respect to a coordinate system associated to }} \\
& \text { reference frame A } \\
& {\left[\tilde{r}^{O P_{i}}\right]_{A} \quad=\text { component vector with respect to reference frame } F_{A} \text { of the skew symmetric }} \\
& \text { tensor } \tilde{r}^{O P_{i}} \\
& {\left[\vec{r}^{P_{2} P_{1}}\right]_{A} \quad=\text { position vector of } P_{1} \text { with respect to } P_{2} \text { with respect to the } \mathrm{SC} F_{A}} \\
& \tilde{r}^{P_{2} P_{1}} \quad=\text { position tensor for vectorial cross-product formulation } \\
& S C \quad=\text { system of coordinates } \\
& \vec{T}_{i} \quad=\text { thrust vector of engine } \mathrm{i} \\
& \Delta \vec{T}_{i} \quad=\text { thrust for control produced by engine } \mathrm{i} \\
& {[\vec{V}]_{A} \quad=\text { components of vector } \vec{V} \text { with respect to } F_{A}} \\
& X_{M} Y_{M} Z_{M} \quad=\text { system of coordinates with respect to } F_{M_{i}}
\end{aligned}
$$

Subscripts

a

e
$=$ aileron

$=$ elevator 


$\begin{array}{ll}\mathrm{f} & =\text { flaps } \\ \mathrm{L} & =\text { "left" in conjunction with the control surfaces } \\ \mathrm{R} & =\text { "right" in conjunction with the control surfaces } \\ \mathrm{r} & =\text { rudder }\end{array}$




\section{Chapter 1: Introduction}

\subsection{Problem Definition}

Aircraft control surface failure accommodation is generally handled by reallocating control authority to other functioning control surfaces (if redundancy exists) through a variety of approaches including both classical ones and artificial intelligence-based techniques. In recent years, significant research efforts have been made to include engine control authority in the aircraft actuator failure accommodation schemes ${ }^{(1)(2)(3)}$. This additional redundancy allows for a more robust failure accommodation mechanism that includes elements of aerodynamic control as well as throttle control for improved performance and fault tolerance. Aircraft upset conditions and their prevention, detection, and accommodation are of high interest because of their relation to aircraft safety. This creates a need for cohesive and wide-ranging systems that solve these problems for pilots, crew, passengers, and cargo. An autonomous system with a high-level knowledge base is preferred due to its ability to automatically adapt to the changing flight conditions within any range of the flight envelope.

Over the past few years several different fault tolerant control models have been developed at West Virginia University (WVU) utilizing the MATLAB/Simulink programming environment and implemented in several aircraft models. These models include an advanced F15 aircraft, a small business jet, and a NASA Generic Transport Aircraft. A Boeing 747 aircraft aerodynamic model was developed for the purposes of this thesis. The WVU aircraft simulation environment uses graphical user interfaces (GUI) that allows for the user to make decisions concerning the pilot input method, flight conditions, failed equipment selection, and engine placement for the flight. The Boeing 747 model includes modeling of nominal flight conditions 
and control surface failures. The goal of this thesis is to develop the foundation work for actuator failure accommodation through use of engine thrust potential and integration into the general failure detection, identification, evaluation, and accommodation (FDIEA) simulation environment.

\subsection{Research Objectives}

The research effort presented in this thesis was aimed at reaching the following objectives:

- Develop and test a large transport aircraft MATLAB/Simulink model at normal and actuator failure conditions that can integrate seamlessly into the current WVU simulation environment.

- Develop and test various control surface actuator failures models.

- Implement and analyze multiple engine aircraft configurations for actuator failure accommodation purposes.

- Investigate the design of a fuzzy logic controller capable of utilizing multiple engines to compensate for control surface failures.

- Analyze the effectiveness and performance of the accommodation scheme.

These research objectives can be summed up in the following personal contributions:

- General framework for using thrust potential in actuator failure accommodation.

- Detailed analysis of twin-engine aircraft and tri-engine aircraft configurations.

- Fuzzy logic controller for engine thrust control. 


\subsection{Thesis Overview}

The following chapter structure describes the organization of this thesis:

- Chapter 2 contains the literature review.

- Chapter 3 introduces the multiple engine model including the reference frames and systems of coordinates used throughout this thesis.

- Chapter 4 illustrates the WVU simulation environment including aircraft model, control system model, and failure types.

- Chapter 5 examines the fuzzy logic control system developed for the purpose of fault tolerant control.

- Chapter 6 discusses the analysis of using an even number and an odd number of engines for aircraft control.

- Chapter 7 presents the analysis of engine compensation when applied to specific actuator failures.

- Chapter 8 describes the simulation results for each type of control surface (aileron, elevator, and rudder) and presents the experimental design including the flight plan and the testing methodology.

- Chapter 9 discusses the conclusions drawn from the results of this research effort and considers areas for further research. 


\section{Chapter 2: Literature Review}

Flight simulators have existed for nearly as long as airplanes have been flying across the sky. The first simulator was created by the Antoinette Company in 1909 for the French Army. This trainer consisted of a half-barrel mounted on a universal joint with flight controls and poles where assistants applied external forces ${ }^{(4)}$. Several other flight simulators utilizing assistants to provide external forces were developed during World War I in the UK and US and even included models designed for air gunnery ${ }^{(5)}$. The first flight simulator not employing assistants for the external forces required was the Link Trainer produced by Edwin Link in New York ${ }^{(6)}$. His design used a pneumatic platform guided by inflatable bellows to provide roll and pitch cues. Digital computers were not introduced to flight simulation until the 1960s, becoming universally accepted in the 1980s. As the computational power of computers increased, the complexity of the flight simulators has increased such that realistic visual and motion cues can be generated for nominal flight conditions as well as a variety of sub-system failures. The current generation of flight simulators is capable of replicating the aircraft's cockpit and flight characteristics in a fully immersed environment for training in the latest wide-body, narrow-body, and rotary-wing $\operatorname{aircraft}^{(7)}$.

\subsection{Modeling and Simulation of Actuator Failures}

Aircraft actuator failures or malfunctions have been identified throughout the years as a leading source of accidents for all classes of aircraft ${ }^{(8)(9)(10)(11)}$. Significant research efforts have recently been directed towards developing fault tolerant control laws that can accommodate actuator failures $^{(12)(13)(14)(15)(16)}$ and increase the safety of aircraft operation. Actuator failures can be completely compensated if enough control redundancy is available. 
Further research has been undertaken at West Virginia University (WVU) in developing a simulation environment for the design and evaluation of direct and indirect adaptive flight control laws with built-in fault tolerant capabilities ${ }^{(17)}$. This system is designed to accommodate aerodynamic control surface failures and critical sensor failures through artificial neural network augmentation and control re-distribution among healthy actuators. However, the additional control redundancy and the use of the aircraft engines as control mechanisms were not investigated.

Actuator failures have typically been modeled by either modeling a mechanism failure, resulting in a locked or unresponsive surface, or through destruction or deformation of the control surface, causing a loss of efficiency of the surface. An actuator mechanism failure does not alter the aerodynamic properties of the control surface but each surface in the pair (left and right) will have different deflections. The resulting forces and moments must be calculated individually such that the separate control surface contributions are isolated. A control surface failure resulting in deformation or destruction may alter the aerodynamic properties of the surface. In modeling this type of failure, it is generally assumed that the aerodynamic properties of the failed surface are proportional to the properties of a healthy surface ${ }^{(17)}$.

\subsection{Engine Use in Actuator Failure Accommodation}

Control system failure compensation is vital for aircraft flight control because of the number of incidents that have occurred owing to such failures and their serious consequences ${ }^{(18)}$. Hence, it is necessary to design flight control systems that are capable of compensating for failures during flight while maintaining the significant flight control. Because of this, multiple efforts have been made in the design and development of fault-tolerant flight control systems. In addition to utilizing additional control surfaces for fault-tolerant control, aircraft propulsion 
systems can be employed for fault-tolerant control. Depending on the number and location, aircraft engines can provide some level of control redundancy that under certain conditions can be critical. This capability has been investigated only in a limited manner ${ }^{(19)(20)(2)(3)(21)(22)}$.

One such endeavor was undertaken by NASA on its Propulsion Controlled Aircraft System. This system was able to utilize differential engine thrust and lead the development of this type of emergency control system. It was flight tested on an MD-11 aircraft in $1997^{(2)}$. Additional methods for control with actuator failures are based on multiple-model, switching and tuning (23) (24) (25) as well as indirect and direct adaptive control algorithms ${ }^{(26)(27)}$ have been developed with varying degrees of success for several different categories of aircraft. Gopinathan, et al., evaluated their multiple-model predictive control scheme on an F/A-18A aircraft during a carrier landing maneuver under actuator control failures ${ }^{(24)}$. They found that this method of accommodation was well suited for the aircraft and the landing maneuver when the stabilator was locked at an imposed position. Aileron and rudder failure situations were not examined. Bošković and Mehra examined a similar multiple-model control strategy for a tailless advanced fighter aircraft in the presence of wing battle damage ${ }^{(23)}$. Their simulation approach examined frozen or stuck control surface actuators. There are several disadvantages of using a multiple-model control strategy. The first is the simulation cost of executing multiple models simultaneously. This comes in the form of a larger computational power and data handling requirements on the control system. The second drawback is the consistency cost of maintaining uniformity among concurrent models. Traditionally, this is handled using some form interpolation between the various models from which the control system is operating. This places constraints on the system designer how many models are necessary to provide acceptable failure accommodation outcomes ${ }^{(28)}$. Liu, et al., approached engine compensation using an 
alternative approach based on a direct adaptive control approach that utilized engine thrust and aileron control for in-flight control system adaptation to rudder and aileron actuator failures ${ }^{(29)}$. This method proved successful for small deflection angle actuator failures from the aircraft's nominal trim position ${ }^{(1)}$.

Within this thesis a novel control scheme for actuator failure accommodation utilizing engine thrust differential was designed by applying FLC principles. The FLC was selected due to its robustness and small computational footprint and system demands ${ }^{(30)}$. The proposed model is capable of lateral, longitudinal, and directional flight control for any single actuator failure within its control limits. Furthermore, the model could be utilized during the design phase of an aircraft in order to locate the positions of the engines to accommodate a given failure or to size the engines when the locations are fixed. This creates a model that is unique in its flexible design and simulation capabilities. Preliminary results were presented at the American Institute of Aeronautics and Astronautics Guidance, Navigation, and Control Conference in Portland, Oregon in August of $2011^{(31)}$. A similar paper on the development of a simulation environment for supporting aircraft health management education was presented at the same joint conference for the Atmospheric Flight Mechanics Conference ${ }^{(32)}$. 


\section{Chapter 3: Multiple Engine Modeling for Fault Tolerant Control Purposes}

\subsection{Reference Frames and Systems of Coordinates}

The reference frames (RF) and systems of coordinates (SC) associated to them used within this paper are as follows:

Earth reference frame denoted by $F_{E}$ is assumed to be inertial. The associated system of coordinates $O_{E} X_{E} Y_{E} Z_{E}$ is defined with the origin at an arbitrary reference point on the surface of the Earth, with $O_{E} Z_{E}$ along the local vertical, positive down, $O_{E} X_{E}$ arbitrarily oriented within the flat surface of the Earth and $O_{E} Y_{E}$ perpendicular following the right-hand rule.

Body reference frame denoted by $F_{B}$ is fixed with respect to the aircraft, which is assumed to be a rigid body. The system of coordinates $O X Y Z$ has the origin at the center of mass of the vehicle with $O Z$ along the local vertical, positive down, $O X$ perpendicular and oriented such that the positive goes through the nose of the aircraft, and $O Y$ perpendicular following the right-hand rule. The orientation of the three axis with respect to $F_{E}$ is defined by the Euler angles $\varphi, \theta, \psi$. The position of the body reference frame on the aircraft can be seen in Figure 3-1.

Engine \#i reference frame denoted by $F_{T_{i}}$ is defined for each aircraft engine. The system of coordinates $P_{i} X_{T_{i}} Y_{T_{i}} Z_{T_{i}}$ is assumed to have its origin at the center of mass of the engine, which also corresponds to the point of application of the thrust. The orientation of $P_{i} X_{T_{i}}$ is assumed along the thrust vector and along the engine axis of symmetry. The engines are assumed to be cylindrical. The orientation of the engine $\mathrm{SC}$ is generally defined with respect to $F_{B}$ by corresponding Euler angles for each engine: $\theta_{i}$ and $\psi_{i}$. 


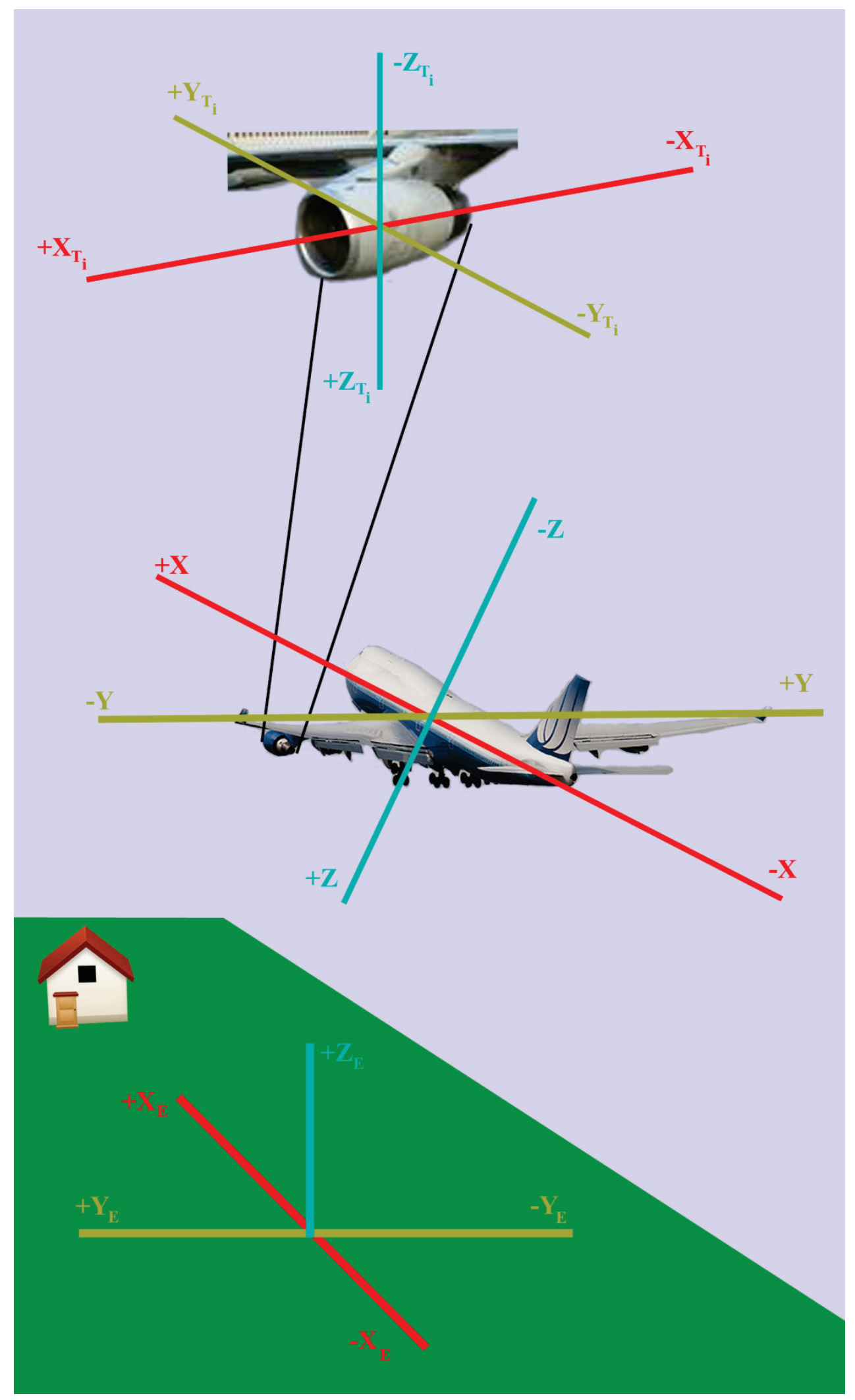

Figure 3-1: Aircraft Tri-View with the Body Reference Frame 


\subsection{Vector Notations and Transformations}

The components of a vector $\vec{V}$ with respect to a SC in RF $F_{A}$ are denoted as:

$$
[\vec{V}]_{A}=\left[\begin{array}{lll}
v_{x} & v_{y} & v_{z}
\end{array}\right]_{A}^{T}
$$

Let the components of the same vector in another $\mathrm{SC} F_{B}$ be:

$$
[\vec{V}]_{B}=\left[\begin{array}{lll}
v_{x} & v_{y} & v_{z}
\end{array}\right]_{B}^{T}
$$

then:

$$
[\vec{V}]_{A}=L_{A B}[\vec{V}]_{B}
$$

where $L_{A B}$ is a $3 \times 3$ transformation matrix depending on the trigonometric functions of the orientation angles $\varphi, \theta, \psi$ of the $\mathrm{CS}$ in $F_{B}$ with respect to the $\mathrm{CS}$ in $F_{A}$.

The position vector of point $P_{1}$ with respect to point $P_{2}$ (origin of vector arrow is at $P_{2}$ ) is denoted as $\vec{r}^{P_{2} P_{1}}$ and its components with respect to SC $F_{A}$ are:

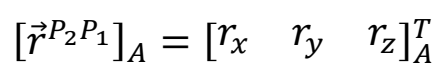

The associated tensor $\tilde{r}^{P_{2} P_{1}}$ is defined such that for an arbitrary vector $\vec{V}$ there is:

$$
\vec{r}^{P_{2} P_{1}} \times \vec{V}=\tilde{r}^{P_{2} P_{1}} \cdot \vec{V}
$$

and in components with respect to $F_{A}$ :

$$
\left[\vec{r}^{P_{2} P_{1}} \times \vec{V}\right]_{A}=\left[\tilde{r}^{P_{2} P_{1}}\right]_{A} \cdot[\vec{V}]_{A}=\left[\begin{array}{ccc}
0 & -r_{z} & r_{y} \\
r_{z} & 0 & -r_{x} \\
-r_{y} & r_{x} & 0
\end{array}\right]_{A} \cdot\left[\begin{array}{c}
V_{x} \\
V_{y} \\
V_{z}
\end{array}\right]_{A}
$$




\subsection{Engine Force and Moment Compensation}

The moment produced by one engine that can be used for control can be expressed as:

$$
\vec{M}_{e i}=\vec{r}^{O P_{i}} \times \Delta \vec{T}_{i}
$$

where $\vec{r}^{O P_{i}}$ is the position vector of the engine center of mass with respect to the aircraft center of mass and $\Delta \vec{T}_{i}$ is the amount of thrust available for control.

Let the moment produced by the failure of one aerodynamic surface be $\vec{M}_{F j}$. To achieve failure accommodation, the total moment for control produced by all engines must balance the total moment produced by all failed surfaces:

$$
\sum_{i=1}^{n} \vec{M}_{e i}=\sum_{j=1}^{m} \vec{M}_{F j}
$$

Assume that the aerodynamic control surface $\mathrm{j}$ is stuck at a deflection $\delta_{j F}$. The moment due to the failure is:

$$
\vec{M}_{F j}=\frac{d \vec{M}}{d \delta_{j}}\left(\delta_{j C}-\delta_{j F}\right)
$$

where $\delta_{j C}$ is the commanded deflection of control surface $\mathrm{j}$, and $\frac{d \vec{M}}{d \delta_{j}}$ represents the derivative of the aerodynamic moment vector with respect to aerodynamic control surface deflection. The components of this vector with respect to body axes are:

$$
\left[\frac{d \vec{M}}{d \delta_{j}}\right]_{B}=\left[\begin{array}{lll}
\frac{d M_{x}}{d \delta_{j}} & \frac{d M_{y}}{d \delta_{j}} & \frac{d M_{z}}{d \delta_{j}}
\end{array}\right]_{B}^{T}
$$


In components with respect to body axes, the total moment due to the failure of possibly several aerodynamic surfaces is given by:

$$
\left[\vec{M}_{F j}\right]_{B}=\left[\frac{d \vec{M}}{d \delta_{j}}\right]_{B}\left(\delta_{j C}-\delta_{j F}\right)
$$

Equation (3.8) can be expressed as:

$$
\begin{aligned}
& \sum_{i=1}^{n}\left[\vec{M}_{e i}\right]_{B}=\sum_{j=1}^{m}\left\{\left[\frac{d \vec{M}}{d \delta_{j}}\right]_{B}\left(\delta_{j C}-\delta_{j F}\right)\right\} \\
& \sum_{i=1}^{n}\left[\vec{r}^{O P_{i}} \times \Delta \vec{T}_{i}\right]_{B}=\sum_{j=1}^{m}\left\{\left[\frac{d \vec{M}}{d \delta_{j}}\right]_{B}\left(\delta_{j C}-\delta_{j F}\right)\right\} \\
& \sum_{i=1}^{n}\left\{\left[\tilde{r}^{O P_{i}}\right]_{B}\left[\Delta T_{i}\right]_{\mathrm{B}}\right\}=\sum_{j=1}^{m}\left\{\left(\delta_{j C}-\delta_{j F}\right)\left[\begin{array}{c}
M_{x \delta_{j}} \\
M_{y \delta_{j}} \\
M_{z \delta_{j}}
\end{array}\right]_{B}\right\} \\
& \sum_{i=1}^{n}\left\{\left[\begin{array}{ccc}
0 & -r_{z} & r_{y} \\
r_{z} & 0 & -r_{x} \\
-r_{y} & r_{x} & 0
\end{array}\right]_{B}\left[\begin{array}{c}
\Delta T_{x i} \\
\Delta T_{y i} \\
\Delta T_{z i}
\end{array}\right]_{\mathrm{B}}\right\}=\sum_{j=1}^{m}\left\{\left(\delta_{j C}-\delta_{j F}\right)\left[\begin{array}{c}
M_{x \delta_{j}} \\
M_{y \delta_{j}} \\
M_{z \delta_{j}}
\end{array}\right]_{B}\right\}
\end{aligned}
$$

From equation (3.15) it can be seen that there are three sets of parameters that need to be evaluated when analyzing the potential of using thrust to compensate for actuator failures. These three sets are:

- Number of engines $\mathrm{n}$ and their locations as determined by the vectors $\vec{r}^{O P_{i}}$;

- Amount of additional thrust available for control from each engine $\Delta \vec{T}_{i}$;

- Severity of the failure as measured by the deflection offset $\left(\delta_{j C}-\delta_{j F}\right)$.

Additionally: 


$$
\left[\begin{array}{l}
\Delta T_{x i} \\
\Delta T_{y i} \\
\Delta T_{z i}
\end{array}\right]_{B}=L_{B F_{i}}\left[\Delta \vec{T}_{i}\right]_{F_{i}}=\left[\begin{array}{c}
\cos \left(\psi_{i}\right) \cos \left(\theta_{i}\right) \\
\sin \left(\psi_{i}\right) \cos \left(\theta_{i}\right) \\
\sin \left(\theta_{i}\right)
\end{array}\right]_{B} \Delta T_{i}
$$

which yields:

$$
\begin{gathered}
\sum_{i=1}^{n}\left\{\left[\begin{array}{ccc}
0 & -r_{i z} & r_{i y} \\
r_{i z} & 0 & -r_{i x} \\
-r_{i y} & r_{i x} & 0
\end{array}\right]_{B}\left[\begin{array}{c}
\cos \psi_{i} \cos \theta_{i} \\
\sin \psi_{i} \cos \theta_{i} \\
\sin \theta_{i}
\end{array}\right] \Delta T_{i}\right\} \\
=\sum_{j=1}^{m}\left\{\left(\delta_{j C}-\delta_{j F}\right)\left[\begin{array}{l}
M_{x \delta_{j}} \\
M_{y \delta_{j}} \\
M_{z \delta_{j}}
\end{array}\right]_{B}\right\}
\end{gathered}
$$

An analysis can be performed by determining the necessary values for some of the parameters when the others are imposed. It can be seen from equation (3.17) that the engine can be used to produce control moments by changing the location of the point of application of the thrust $\left(r_{x i}, r_{y i}\right.$, and $\left.r_{z i}\right)$, by changing the orientation of the thrust vector $\left(\theta_{i}\right.$ and $\left.\psi_{i}\right)$, and/or by changing the magnitude of the thrust available for control $\left(\Delta T_{i}\right)$. The model also allows the determination, given a certain propulsion system configuration, of the severity of the failure that can be handled. 


\section{Chapter 4: WVU Simulation Environment}

\subsection{General Description of the WVU Simulation Environment}

WVU has developed an advanced simulation environment for the purpose of designing, evaluating, and validating aircraft fault-tolerant control laws ${ }^{(17)}$. MATLAB and Simulink are used in order to maximize portability and flexibility for future expansion. The Flight Dynamics and Control (FDC) toolbox ${ }^{(33)}$ within the Simulink library is included for solving the equations of motion and for modeling wind and atmospheric turbulence effects within the simulation. The model is interfaced with an open source flight visualization software package FlightGear ${ }^{(34)}$ in order to provide visual indications. The core modules of the WVU simulation environment are presented in Figure 4-1.

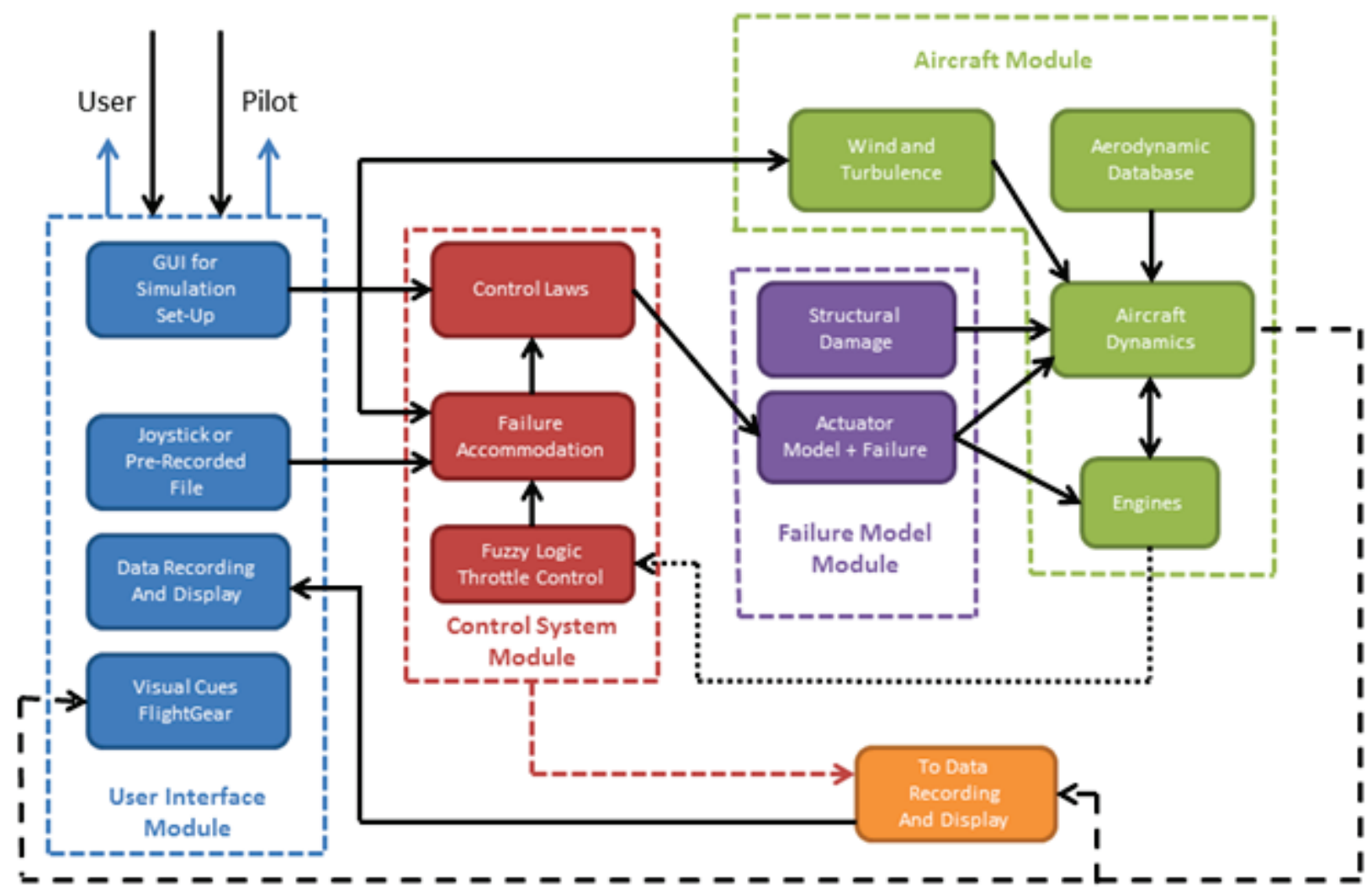

Figure 4-1: General Architecture of the WVU Simulation Environment 
These modules include:

- Aircraft Model Module

- Control System Module

- $\quad$ Aircraft Sub-System Failure Models

- User Interface

The high level Simulink model for the direct desktop configuration is shown in Figure 4-2.

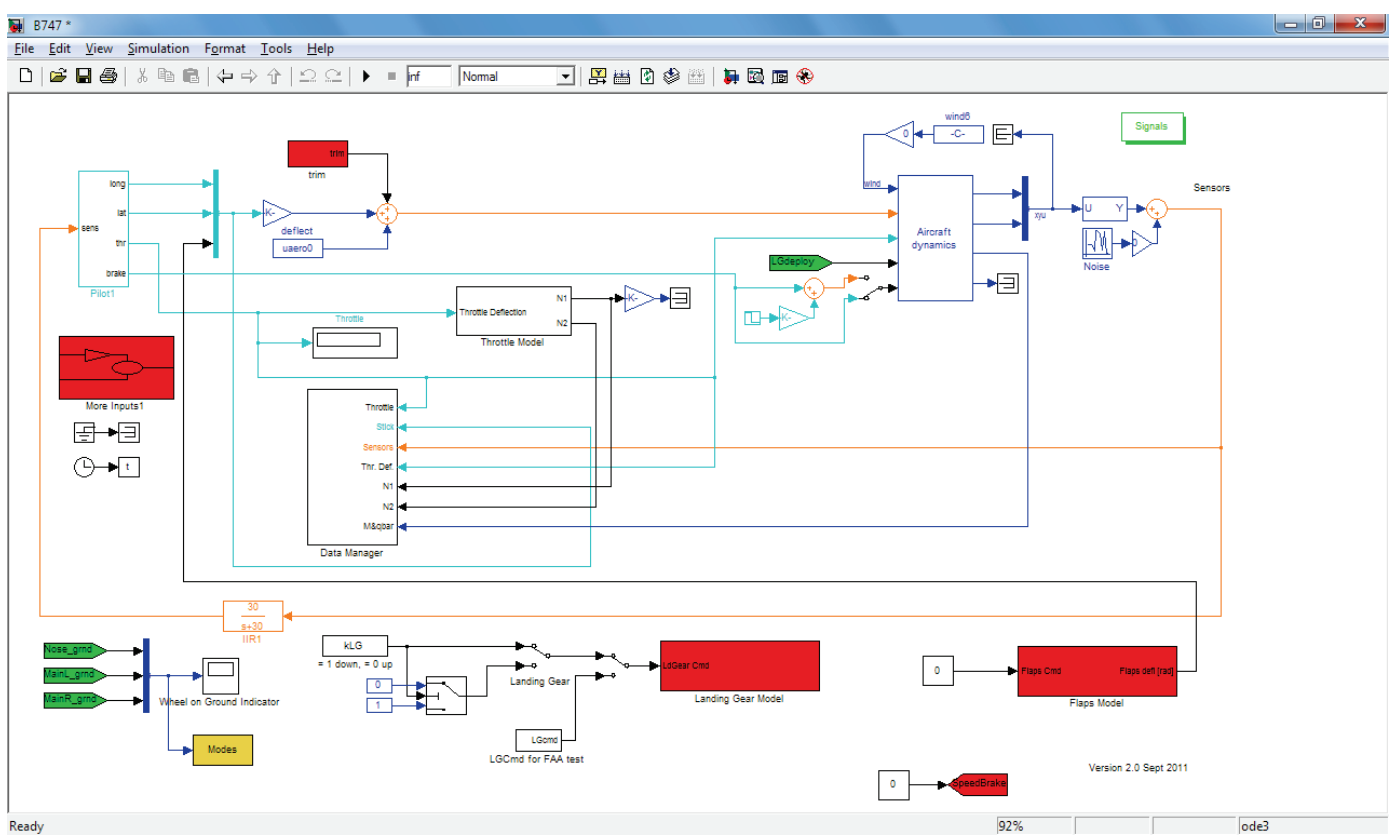

Figure 4-2: Simulink Model of the WVU Simulation Environment

The aircraft model can be flown using a joystick. The GUI menus are used to set the user-configurable values for the desired simulation scenarios such as nominal conditions flight or locked control surface testing. Some of these user-configurable options relate to the failure type and magnitude and other input/output content. An example of the user interface provided by 
FlightGear as well as flight parameter monitoring provided by Simulink scopes can be viewed in Figure 4-3.

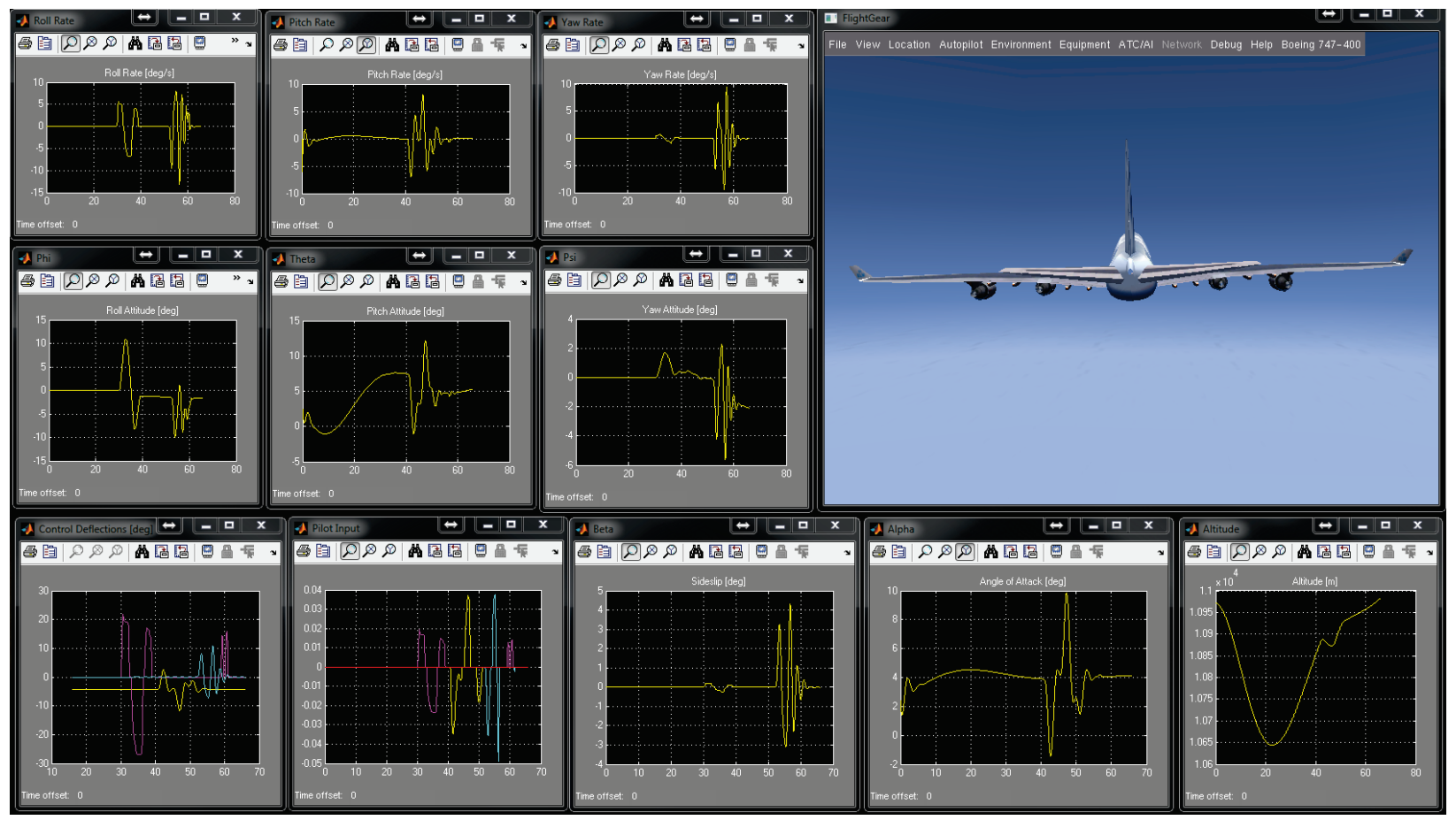

Figure 4-3: WVU Simulation Environment Interface

A multi-engine large transport aircraft model has been developed for the investigation and analysis of utilizing engine thrust to accommodate for aerodynamic control surface failures. The aerodynamic model is based on generic values of the stability and control derivatives ${ }^{(35)}$ for a representative aircraft in this class. This simulation environment allows for the study of two to ten engines at specific locations in relation to the aircrafts center of gravity. The model allows for the determination of engine placement, engine thrust, or maximum control surface deflection failure to be fixed while the remaining parameters are determined. Furthermore, the airplane model is easily adaptable for any other type of fixed wing aircraft. 


\subsection{Aircraft Model}

The Aircraft Model Module contains four main components: wind and turbulence modeling, aerodynamic database, aircraft dynamic equations of motion, and the engines model. The engines model was expanded such that multiple engine configurations could be utilized for analysis. The engine model utilizes generic thrust look-up table based on Mach number, aircraft altitude, and engine rotations per minute.

The turbulence model implemented within the simulation environment is based on the Dryden model. This model uses the Dryden spectral representation to add turbulence to the simulation by passing band-limited white noise. The model is also capable of simulating the effects of constant wind of a pre-determined direction and magnitude. The calculation of aerodynamic forces and moments is distributed for each control surface, wing, and engine. This is necessary for modeling the failures with an adequate level of generality.

\subsection{Sub-System Failure Model}

The sub-system failure models allow for various aspects of the model to fail in a controlled manner such that the dynamics of the failure can be analyzed. There are two types of aerodynamic control surface failure implemented within the model. The first type of failure relates to an actuator mechanism failure. The control surface remains intact and fixed in the current or a user imposed position at post-failure conditions. The second type of failure corresponds to physical destruction of the control surface. It involves reducing the aerodynamic efficiency of the control surface starting at the occurrence of the failure ${ }^{(36)}$. The user is able to set different failure parameters such as the type of failure and magnitude of failure as well as the time of the failure occurrence on one of five control surfaces of the general aircraft model such 
as left or right ailerons, left or right elevators, or the rudder. Formally, the deflection of a control surface that is locked or jammed at a "current" position can be expressed as:

$$
\delta_{e}(t)=\left\{\begin{array}{cl}
\delta_{e}(t) \text { provided by pilot } & \text { if } t<t_{f} \\
\delta_{e}\left(t_{f}\right) & \text { if } t \geq t_{f}
\end{array}\right.
$$

where $t_{f}$ is the moment of occurrence of the failure. If the surface is supposed to move to a user specified position $\delta_{e f}$ and stay there, then the deflection can be expressed as:

$$
\delta_{e}(t)=\left\{\begin{array}{cc}
\delta_{e}(t) \text { provided by pilot } & \text { if } t<t_{f} \\
\delta_{e}\left(t_{f}\right)+\frac{a}{s+a} \delta_{e f} & \text { if } t \geq t_{f}
\end{array}\right.
$$

where first order dynamics are assumed and the time constant must be specified by the user.

If physical destruction or alteration is chosen as the failure parameter, then it is assumed that the alteration of the aerodynamic properties is such that the forces and moments generated by the control surface after the failure differ from those before the failure by a proportional factor $\left(\bar{s}_{d}\right)$ affecting an efficiency parameter $E_{u_{k}}, k=1, \ldots, m$, with $\mathrm{m}$ the total number of control surfaces. An important element of this modeling approach is to define the efficiency parameter $E_{u_{k}}$ such that it characterizes appropriately the nature of the failure. The surface damage parameter $s_{d}$ models the magnitude of the failure through the ratio between the efficiency parameter after and before the failure occurring moment:

$$
s_{d}=\frac{\left(E_{u_{k}}\right)_{\text {AfterFailure }}}{\left(E_{u_{k}}\right)_{\text {BeforeFailure }}}
$$

Therefore, $s_{d} \in[0,1]$, with $s_{d}=1$ for the "no failure" case and $s_{d}=0$ for a failure involving a completely missing surface or a complete loss of "efficiency". 


\subsection{Graphical User Interfaces (GUI)}

The main menu, as seen in Figure 4-4, allows for the user to select the desired simulation. The simulation is capable of modeling nominal flight conditions as well as abnormal flight conditions such as control surface failures.

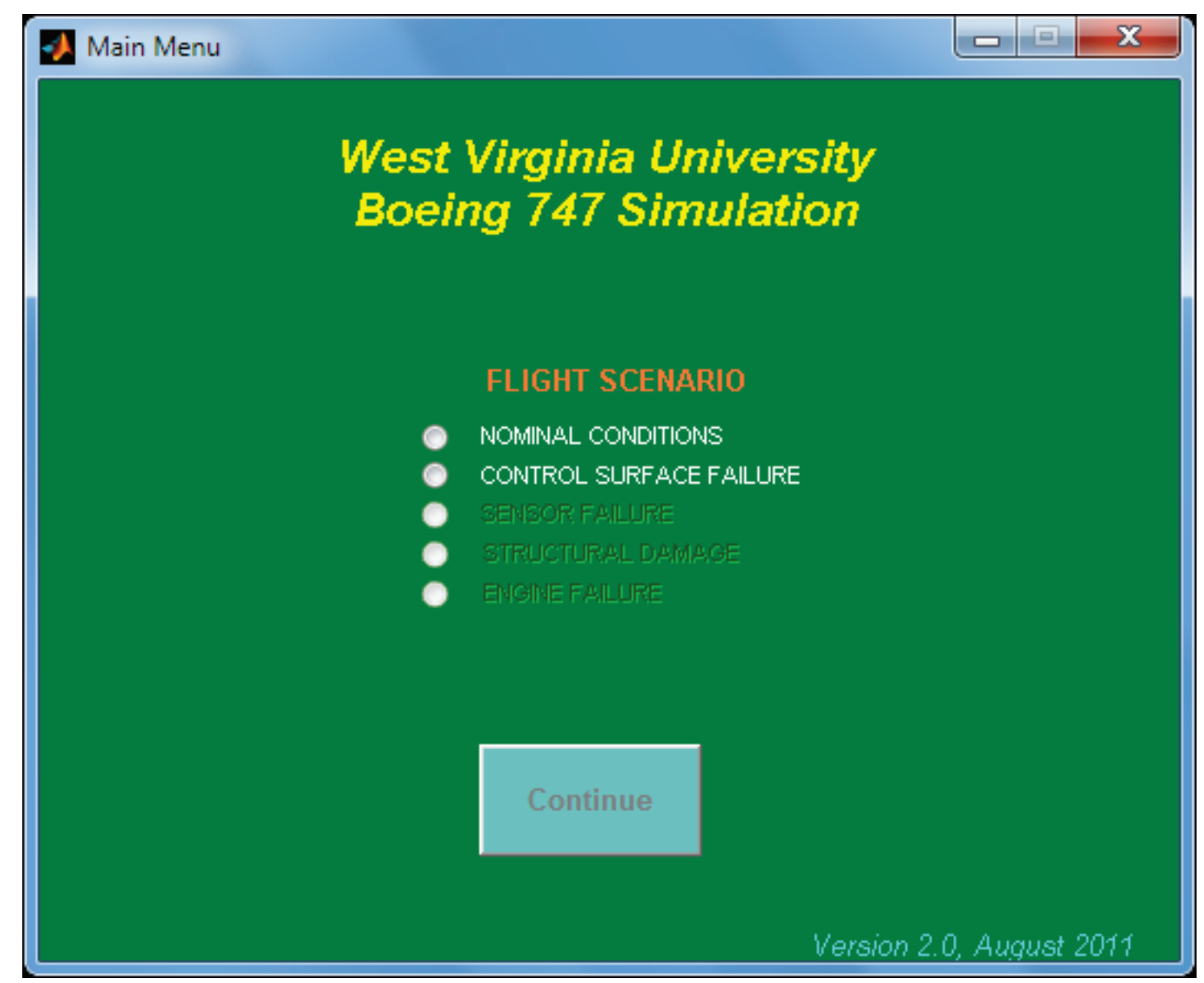

Figure 4-4: Boeing 747 Simulation Main Menu

Different types of pilot input methods can be selected using the menu in Figure 4-5. These input sources include all real-time user input, pre-recorded maneuvers, or any combination of user and pre-recorded data. From the next menu select the initial conditions from Figure 4-6. 


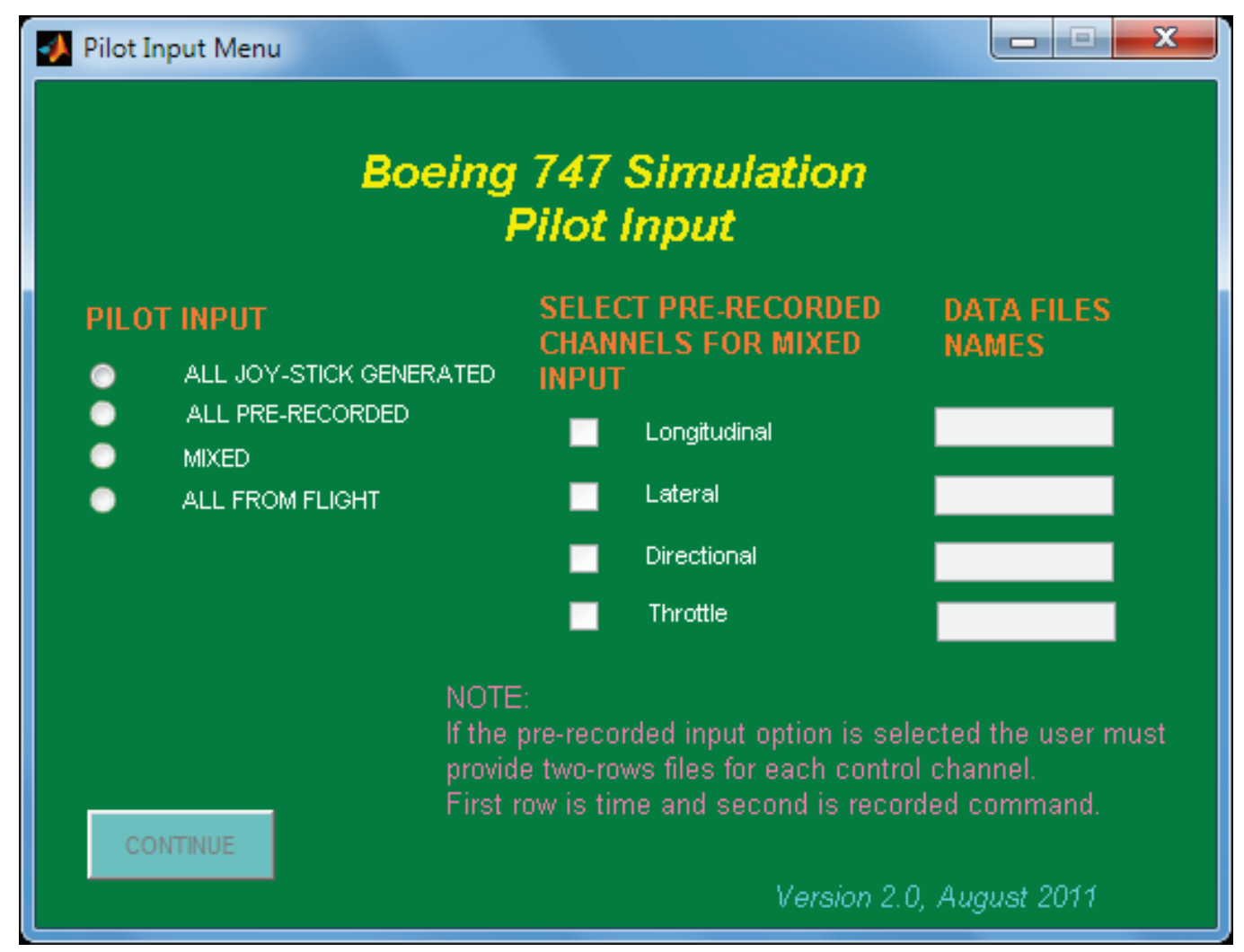

Figure 4-5: Pilot Input Menu

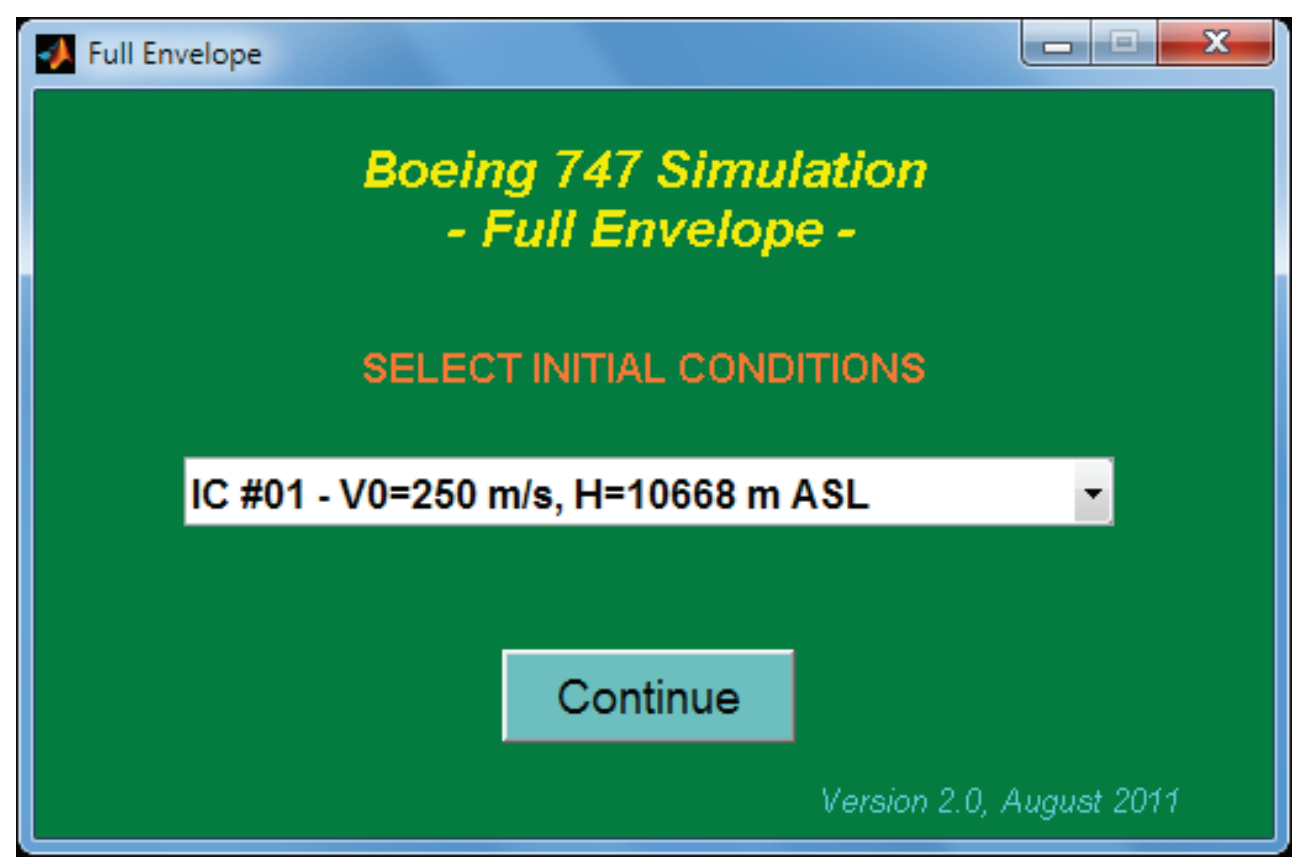

Figure 4-6: Initial Conditions Selection 
If a failure scenario was selected from the Figure 4-4, the Control Surface Failure Conditions Menu will open (Figure 4-7). Select the failure scenario and the failed control surface and enter any additional relevant information in the boxes.

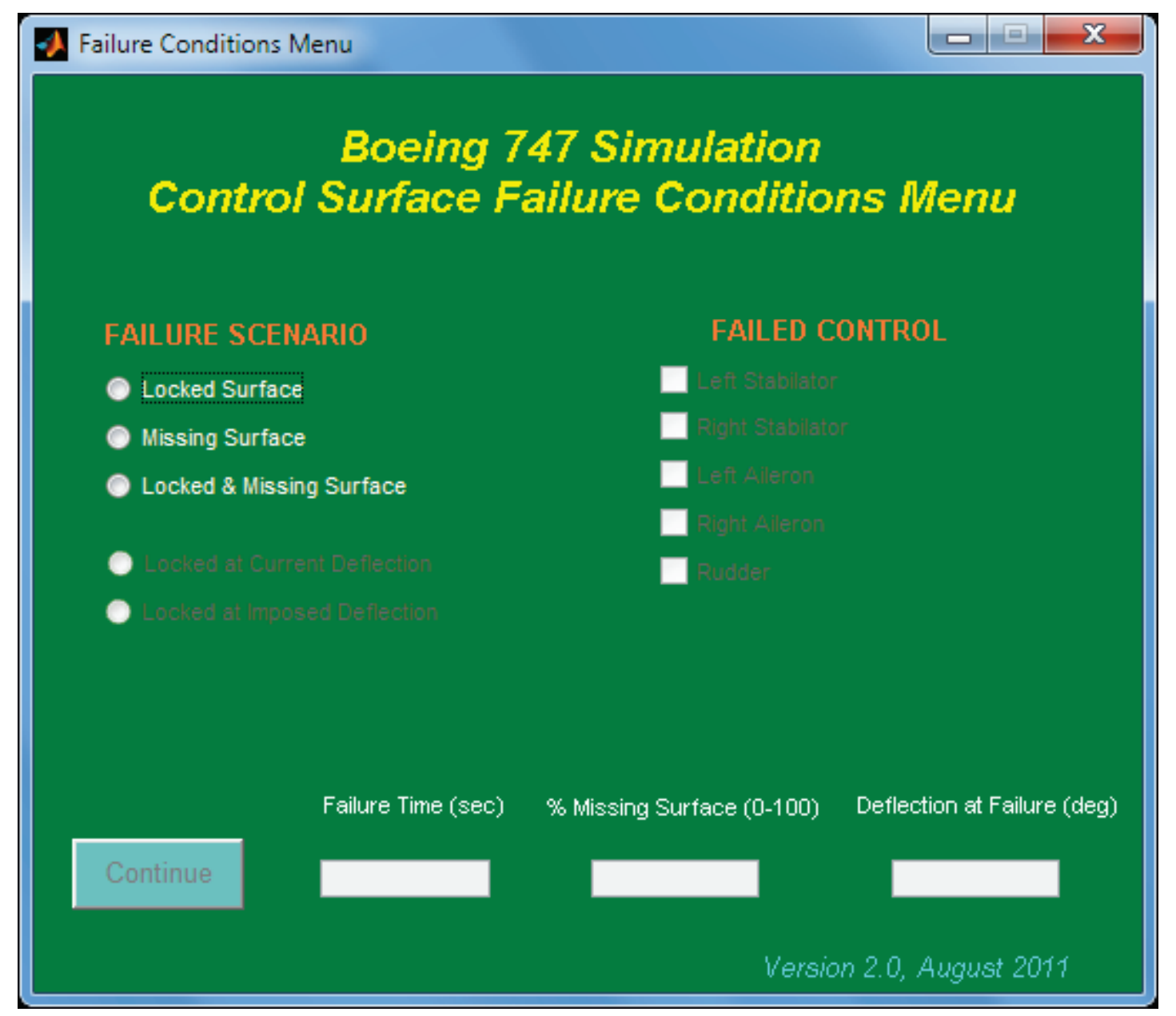

Figure 4-7: Control Surface Failure Conditions Menu

The engine placement menu in Figure 4-8 allows for the selection of different engine configurations. The number of engines can be varied between two and ten. Once the number of engines is selected, the user is able to specify the engine center of gravity location in relation to the aircraft's center of gravity. 


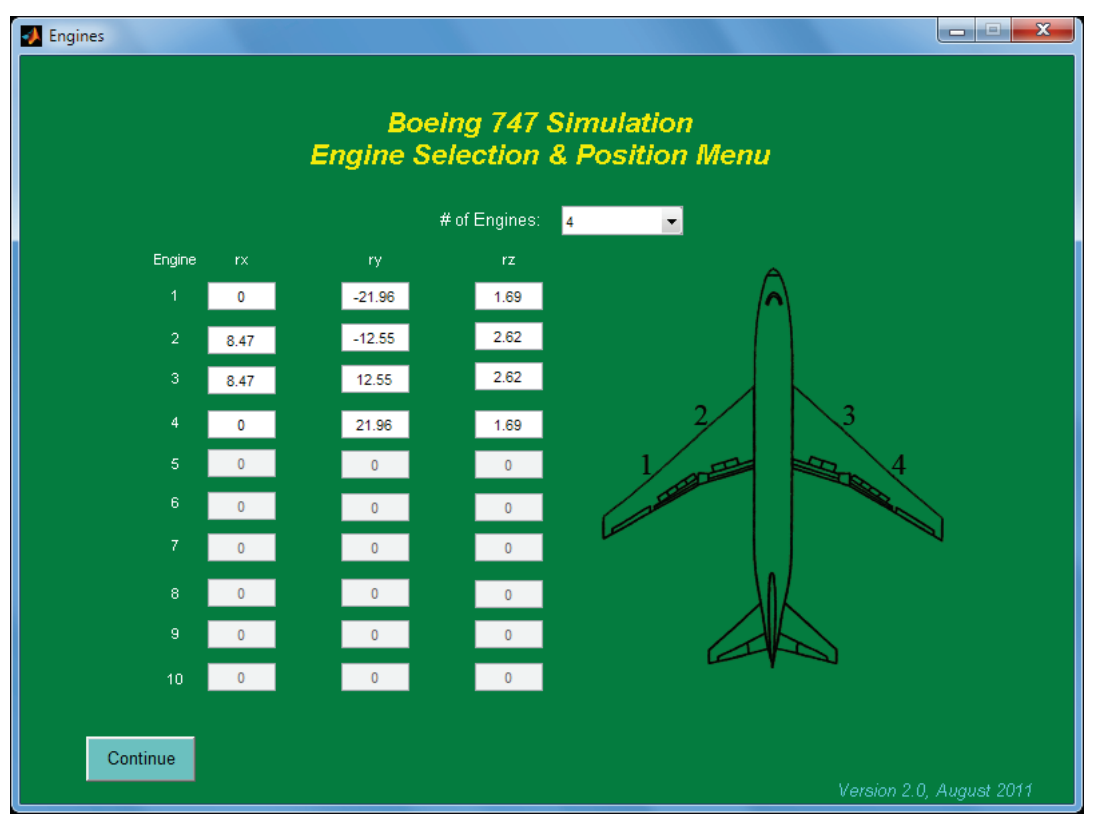

Figure 4-8: Engine Selection and Position Menu

Simulink scopes, as seen in Figure 4-3, allow for the real-time monitoring and analysis of relevant parameters during the simulation and for examination of the histories after the simulation has commenced. These scopes may be selected from Figure 4-9.

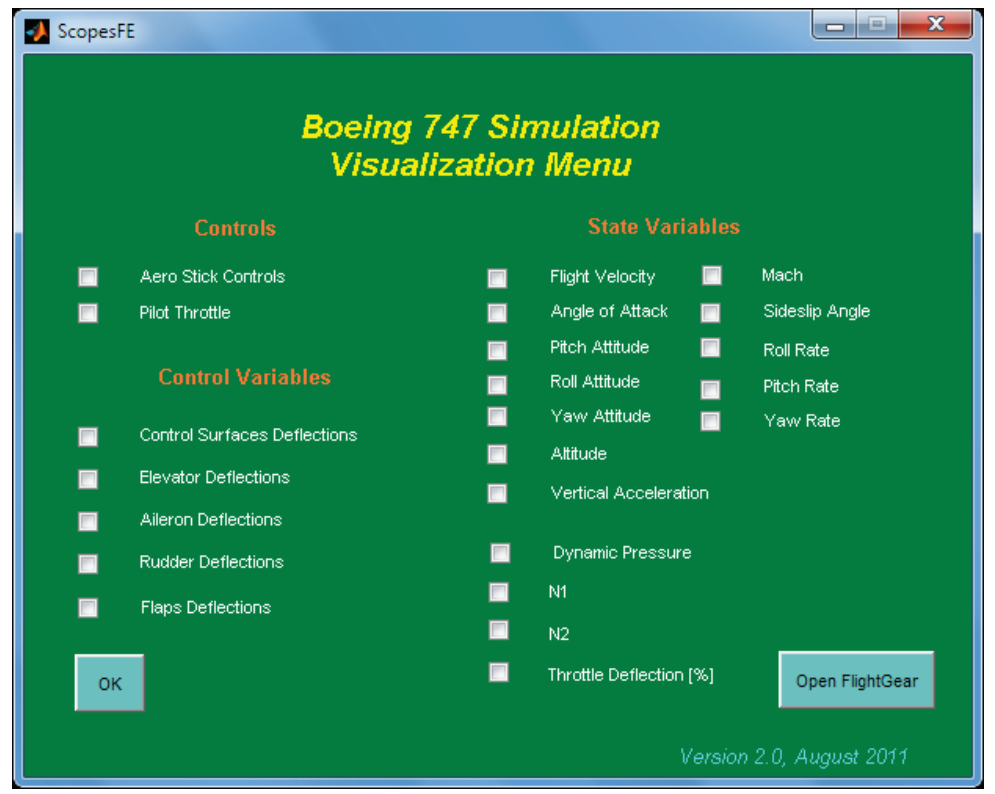

Figure 4-9: Flight Visualization Menu 


\section{Chapter 5: Fuzzy Logic Throttle Controller}

\subsection{Fuzzy Logic Overview}

Fuzzy logic is a form of multi-value logic that operates with statements that can be true, false, or anywhere in between and can be used to model the knowledge, experience, and modus operandi of a human operator ${ }^{(30)}$. Fuzzy logic allows avoiding the rigidity of classic logic and the mathematics associated to it and facilitates modeling of processes characteristic to living matter, such as intelligence. Typically, a human verbally describes cognitive performance related to the control activity in the terms of rules. These rules take the form of a "linguistic variable is a linguistic value" or "control variable is a linguistic value". A linguistic variable is often a "physical variable" such as position, velocity, acceleration, etc. A control variable could be an actuator position, voltage to an actuator, cylinder displacement, etc. The linguistic value is an attribute that is associated to the linguistic variable - or a fuzzy property of the linguistic variable - such as small, very small, large, etc. A collection of such rules describing the control task forms an expert system. The approach is useful when control laws cannot be formulated analytically or when performance and robustness must be improved by including information from human operators.

In general, the flow of a fuzzy logic-based controller consists of three major modules ${ }^{(30)}$ (37): fuzzification, inference engine, and defuzzification. The crisp measurements from the sensors, or linguistic variables, must be converted through the fuzzification process into a fuzzy input that represents the degree to which the crisp measurements belong to each of the fuzzy sets defined by the linguistic variables - an attribute associated with the linguistic variable such as small, very small, large, etc. This takes place in the fuzzification module. Then the fuzzy input is used to create a fuzzy output or command according to the set of condition rules provided by 
an expert operator in the form of "IF - THEN" rules ${ }^{(30)(37)(38)}$. This is equivalent to translating the control rules from natural language into fuzzy logic. The module in which this operation is performed is the inference engine. The fuzzy command represents membership values of the output to fuzzy sets defined by the linguistic values associated with the command. This information must then be converted back into a crisp value, within the defuzzification module, and used directly as command signals.

\subsection{Controller Design}

The FLC throttle control model is composed of three separate FLC systems as seen in Figure 5-1. These controllers correspond to the longitudinal, lateral, and directional control channels. Each FLC consists of two inputs and one output. The input comprises of the pilot input on the individual channels and the respective roll rate, pitch rate, and yaw rate. An example of one of the individual FLC can be examined in Figure 5-2.

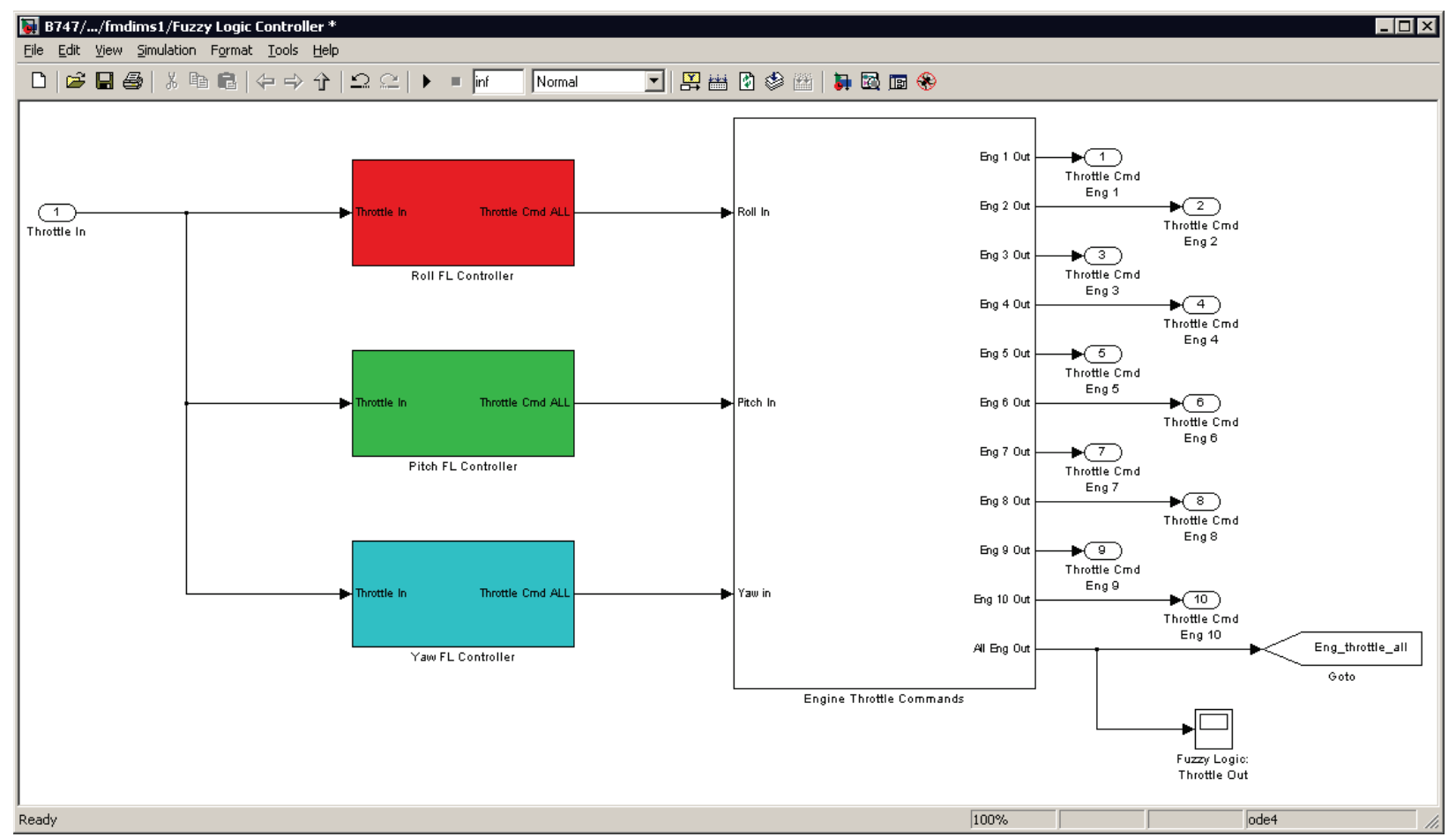

Figure 5-1: Simulink Fuzzy Logic Model Overview 
The crisp inputs from the pilot input and roll rate, pitch rate, and yaw rate sensors must be converted through the fuzzification process into a fuzzy input that represents the degree to which the crisp measurements belong to each of the fuzzy sets defined by the linguistic values. The linguistic values are attributes associated with the linguistic variables such as small, very small, large, etc. All of this takes place in the fuzzification module. The fuzzy inputs are used to create a fuzzy output or command according to the set of "IF - THEN" rules supplied by an expert operator ${ }^{(30)(37)(38)}$. This correlates the natural language set of operators supplied by the experts into the mathematical formulation of the FLC. The fuzzy command represents the membership values of the output to fuzzy sets defined by the linguistic values associated with the command. Finally, this information is transformed into a crisp signal that the controller passes along to the aircraft model by converting the fuzzified output utilizing a process similar but opposite of the fuzzification process.

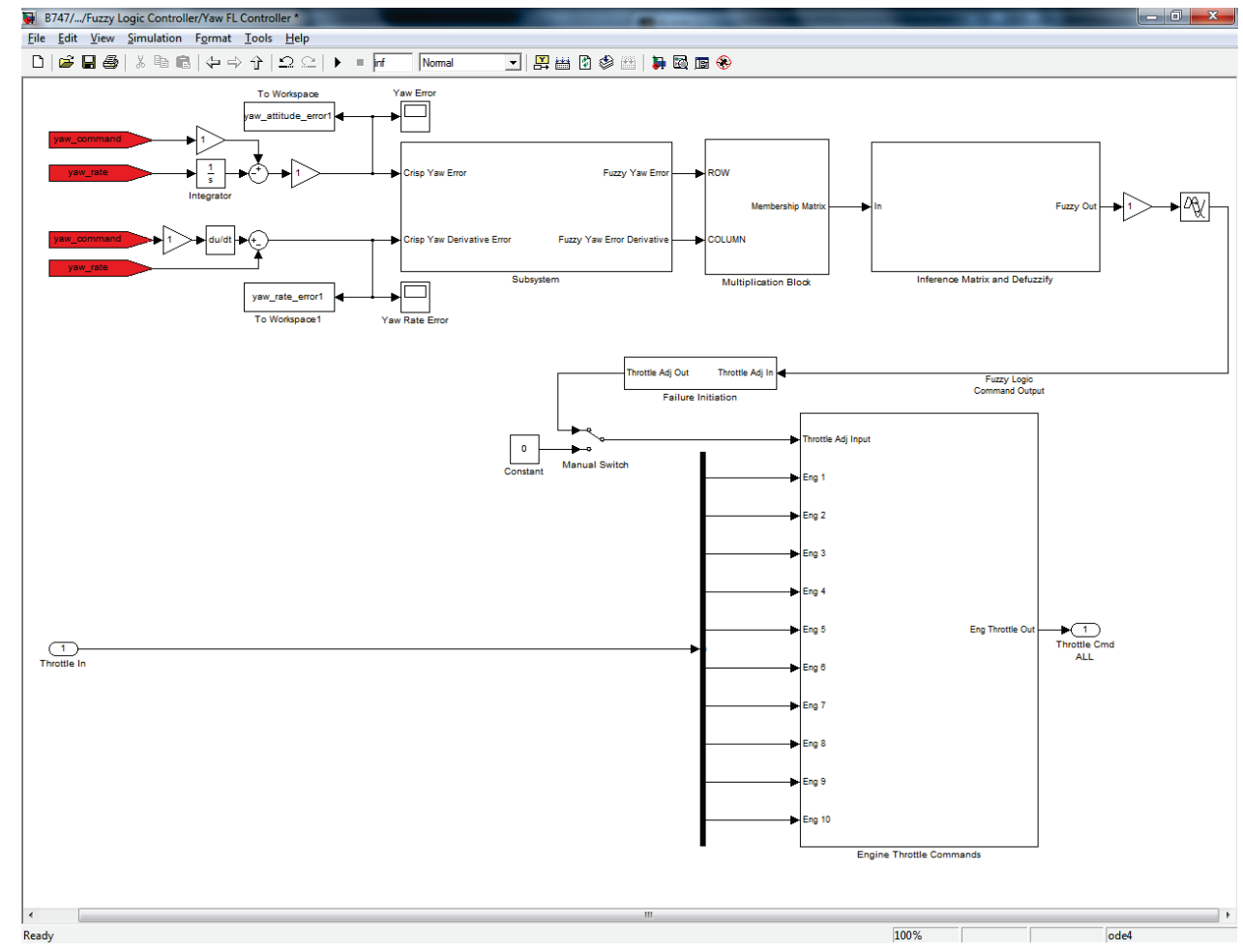

Figure 5-2: Example of a FLC 
A simple FLC was implemented on the WVU large transport 747 model for the purpose of illustrating the fault tolerant capabilities of propulsion devices in the presence actuator failures. The control strategy consists of commanding the attitude angle and angular rate of the three body axes using engine throttle. As a result, each controller has two linguistic variables attitude angle error between the pilot command derivative and the actual angle and the rate of change in this error as defined by (5.1) and (5.2).

\author{
Error Command $=$ Pilot Command - Aircraft Attitude \\ Error Derivative \\ $=$ Pilot Command Derivative \\ - Aircraft Rate of Change
}

The crisp value from each linguistic variable is fuzzified using five linguistic values Large Negative, Negative, Zero, Positive, and Large Positive. There are two types of membership functions utilized - a trapezoidal shape and a triangular shape. For the attitude angle error, both trapezoidal and triangular membership functions were used, while for the rate error, only trapezoidal membership functions were utilized. The membership functions for the inputs for roll, pitch, and yaw can be viewed in Figure 5-3, Figure 5-4, and Figure 5-5. The output command uses triangular shaped functions. See Figure 5-6 for the output membership functions and surface plot. 

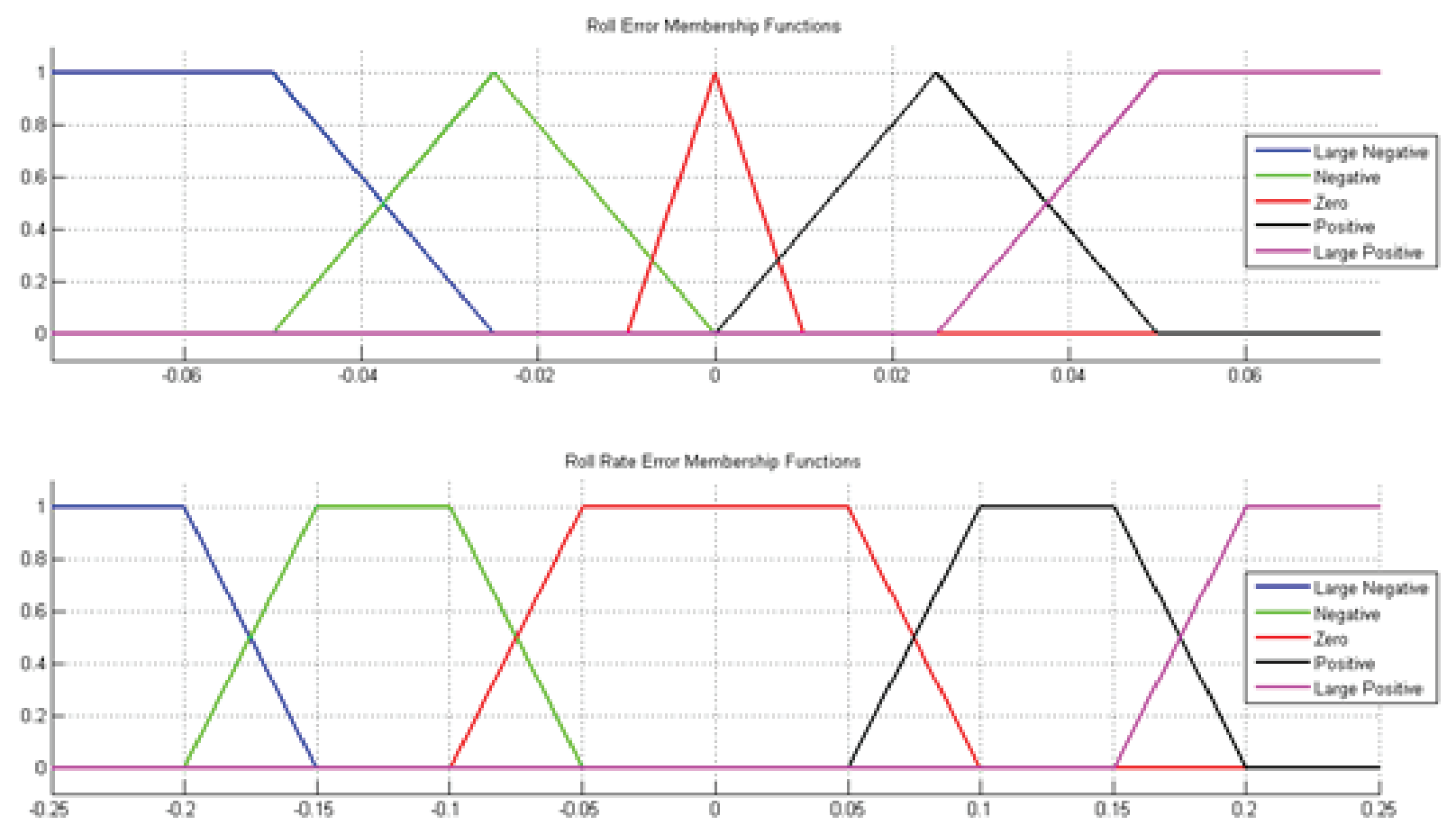

Figure 5-3: Roll Membership Functions
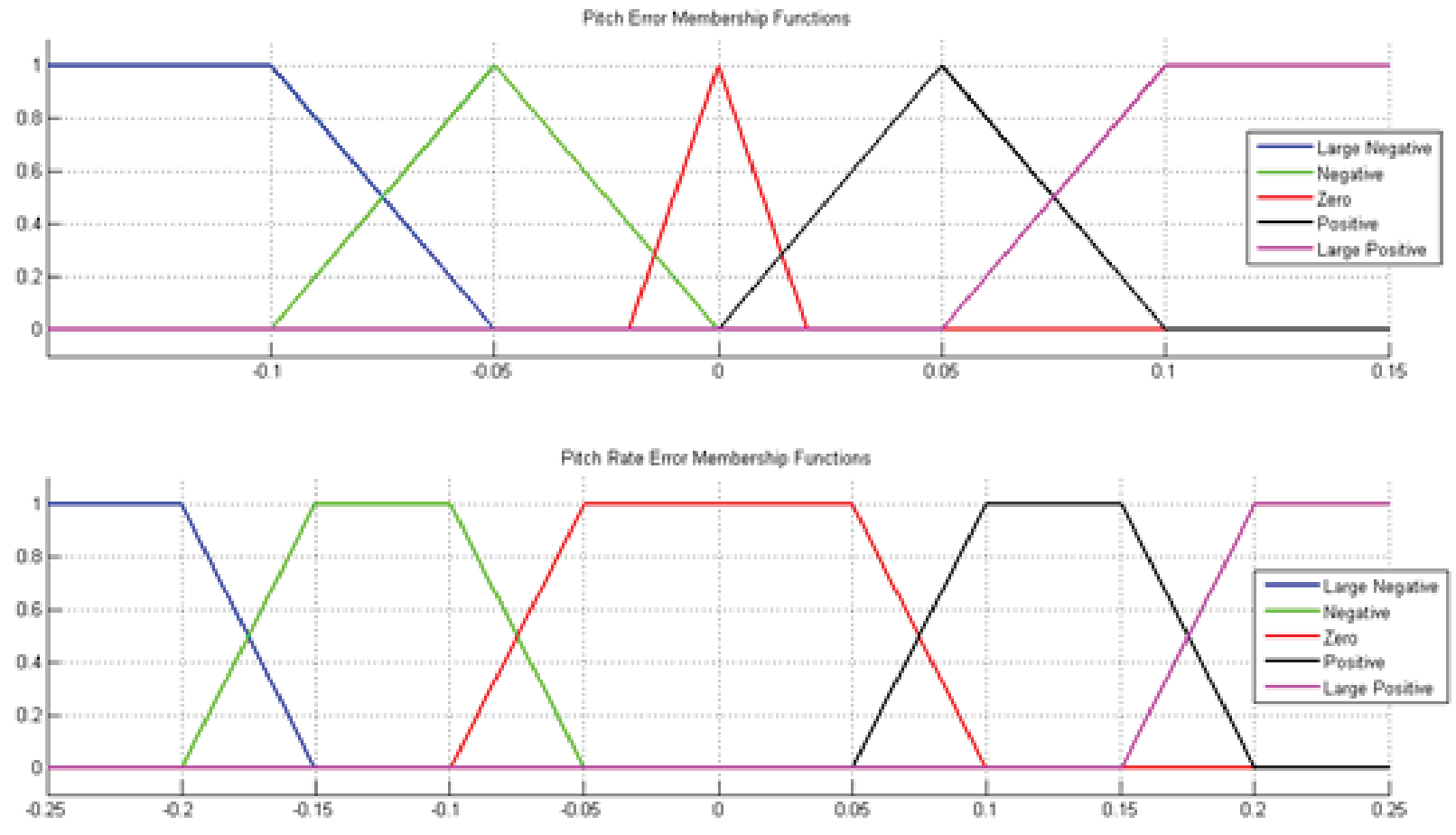

Figure 5-4: Pitch Membership Functions 

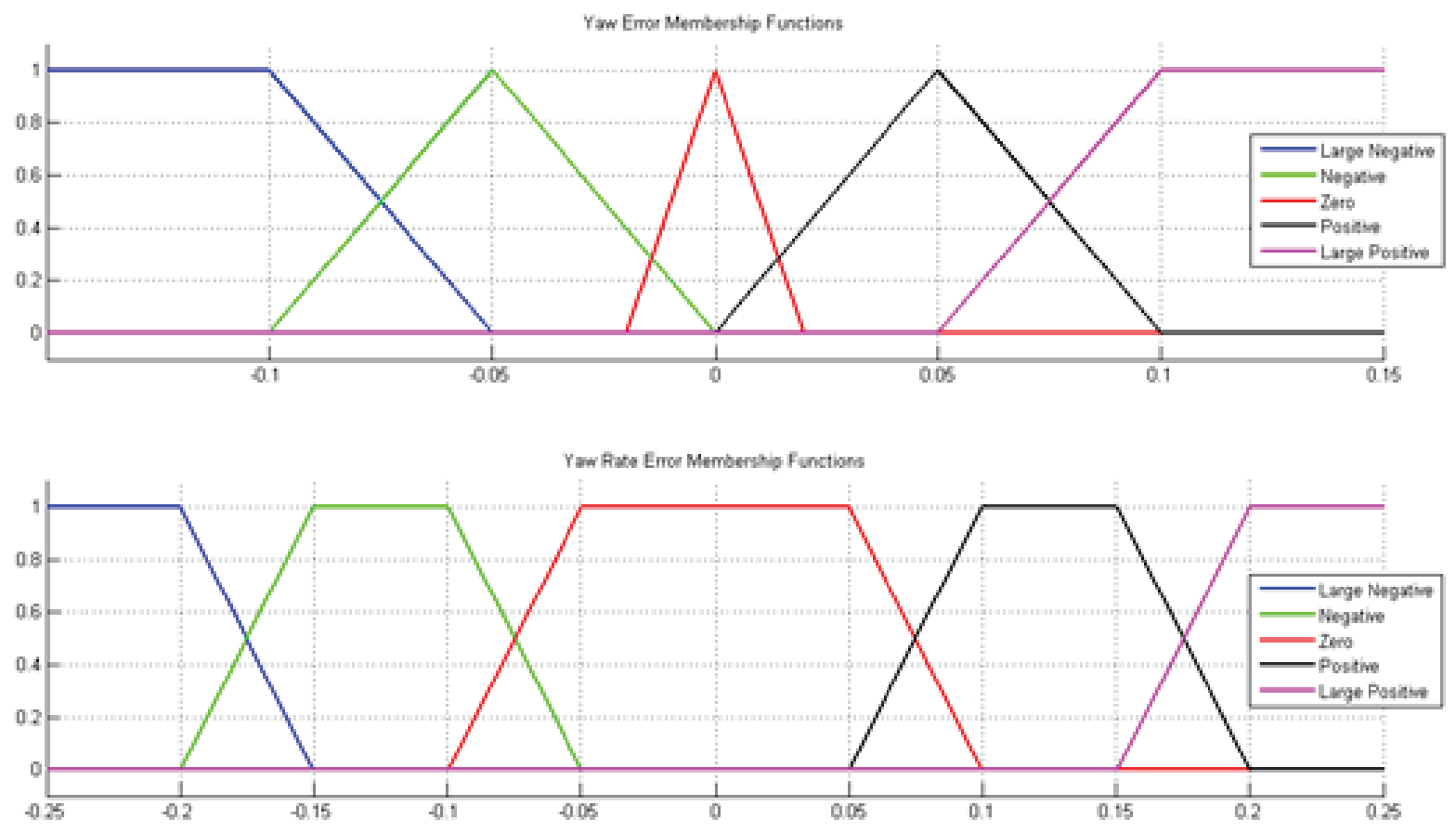

Figure 5-5: Yaw Membership Functions
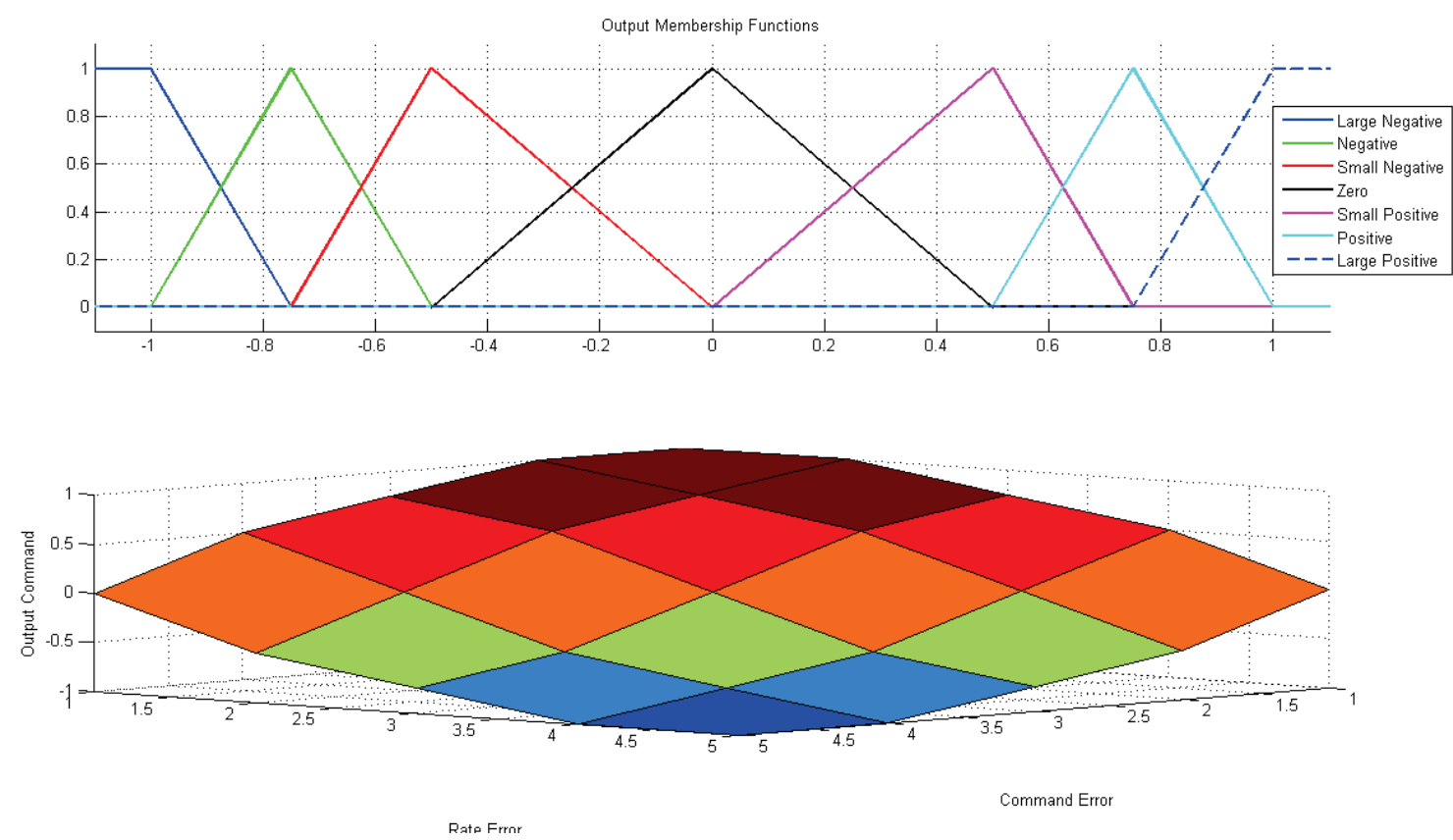

Figure 5-6: Output Membership Function for Roll, Pitch, and Yaw Channels 
The two 5-dimensional input linguistic variable vectors produce a five-by-five grid for all possible combinations. This grid is used to formulate the inference rule matrix presented in Table 5-1 for the lateral, longitudinal, and directional channels. The output linguistic variable is additional throttle deflection and can be composed of seven linguistic values - Large Negative, Negative, Small Negative, Zero, Small Positive, Positive, and Large Positive. For example, cell $(4,2)$ corresponds to the following inference rule: if the angle error is "Positive" and the rate error is "Negative", then the additional throttle deflection must be "Zero". Within the inference engine module, the fuzzy command is generated for each engine with consideration given to its relative position to the center of gravity of the aircraft. The output from the inference engine is passed into the defuzziciation module where the fuzzy values from the inference matrix are converted into a crisp throttle alteration command. This command is then sent into an additional module that alters each individual engine throttle command. The pilot throttle command is assumed to be the same for each engine. The alteration from the FLC either increases or decreases this value based on the engine's location with respect to the aircraft's center of gravity and the required compensating moment.

Table 5-1: Output Inference Rules Matrix

\begin{tabular}{|c|c|c|c|c|c|}
\hline $\begin{array}{c}\text { Error } \\
\text { Rate }\end{array}$ & Large Negative & Negative & Zero & Positive & Large Positive \\
\hline Large Negative & Large Positive & Large Positive & Positive & Small Positive & Zero \\
\hline Negative & Large Positive & Positive & Small Positive & Zero & Small Negative \\
\hline Zero & Positive & Small Positive & Zero & Small Negative & Negative \\
\hline Positive & Small Positive & Zero & Small Negative & Negative & Large Negative \\
\hline Large Positive & Zero & Small Negative & Negative & Large Negative & Large Negative \\
\hline
\end{tabular}




\subsection{Implementing the Fuzzy Logic Controller}

The FLC can be implemented in many different aircraft models. The controller utilizes the pilot stick commands and the aircraft's measured attitude and angular rates. The net result of the controller is an alteration of the throttle position to each engine after a failure has occurred. This control could be modified such that it controls the engine RPM, fuel flow rate, or any other single engine property. The steps necessary to implement the FLC into an aircraft are as follows:

1. Copy the FLC block into the Engine Management section of the aircraft model.

2. The input gains for the roll, pitch, and yaw controllers need to me modified such that the pilot commands and the respective attitude and angular rate are roughly normalized.

3. The output gains for each engine need to be modified. These gains for the roll and yaw controllers are designed to favor the outboard engines over the inboard engines because of the greater moment arm that they possess. For the pitch controller, the gains are intended to favor the engines that are farthest from the XY-plane. For the three controllers, the gains that are the largest are normalized with the other gains being less than one in proportion to their location to the aircraft's center of gravity.

Additional modifications may need to be implemented concerning the number of throttle inputs into the system. The FLC is designed to accommodate between two and ten engines. Depending on the number of engines that the aircraft has, the number of throttle inputs may need to be altered. The controller must have ten throttle input values between zero and one. For an aircraft with two engines, the first two values will be the pilot throttle values and the other eight must be set as a constant zero. If the aircraft has four engines, then the first four inputs correspond to the pilot input commands and the remaining six are set at a constant zero. 


\section{Chapter 6: Analysis of Engine Models}

The overall governing equation (3.17) is:

$$
\begin{gathered}
\sum_{i=1}^{n}\left\{\left[\begin{array}{ccc}
0 & -r_{i z} & r_{i y} \\
r_{i z} & 0 & -r_{i x} \\
-r_{i y} & r_{i x} & 0
\end{array}\right]_{B}\left[\begin{array}{c}
\cos \psi_{i} \cos \theta_{i} \\
\sin \psi_{i} \cos \theta_{i} \\
\sin \theta_{i}
\end{array}\right]_{B} \Delta T_{i}\right\} \\
=\sum_{j=1}^{m}\left\{\left(\delta_{j C}-\delta_{j F}\right)\left[\begin{array}{c}
M_{x \delta_{j}} \\
M_{y \delta_{j}} \\
M_{z \delta_{j}}
\end{array}\right]_{B}\right\}
\end{gathered}
$$

When the moment due to the difference between commanded and actual deflection at failure is known, one can denote the total moment that requires compensation as:

$$
\sum_{j=1}^{m}\left\{\left(\delta_{j C}-\delta_{j F}\right)\left[\begin{array}{l}
M_{x \delta_{j}} \\
M_{y \delta_{j}} \\
M_{z \delta_{j}}
\end{array}\right]_{B}\right\}=\left[\begin{array}{l}
M_{x_{T}} \\
M_{y_{T}} \\
M_{z_{T}}
\end{array}\right]
$$

Equation (6.1) can then be re-written as:

$$
\sum_{i=1}^{n}\left\{\left[\begin{array}{ccc}
0 & -r_{i z} & r_{i y} \\
r_{i z} & 0 & -r_{i x} \\
-r_{i y} & r_{i x} & 0
\end{array}\right]_{B}\left[\begin{array}{c}
\cos \psi_{i} \cos \theta_{i} \\
\sin \psi_{i} \cos \theta_{i} \\
\sin \theta_{i}
\end{array}\right] \Delta T_{i}\right\}=\left[\begin{array}{c}
M_{x_{T}} \\
M_{y_{T}} \\
M_{z_{T}}
\end{array}\right]
$$

\subsection{Even Engine Number Model Example}

When $n=2$, equation (6.3) becomes: 


$$
\begin{aligned}
& {\left[\begin{array}{ccc}
0 & -r_{1 z} & r_{1 y} \\
r_{1 z} & 0 & -r_{1 x} \\
-r_{1 y} & r_{1 x} & 0
\end{array}\right]\left[\begin{array}{c}
\cos \psi_{1} \cos \theta_{1} \\
\sin \psi_{1} \cos \theta_{1} \\
\sin \theta_{1}
\end{array}\right] \Delta T_{1}} \\
& +\left[\begin{array}{ccc}
0 & -r_{2 z} & r_{2 y} \\
r_{2 z} & 0 & -r_{2 x} \\
-r_{2 y} & r_{2 x} & 0
\end{array}\right]\left[\begin{array}{c}
\cos \psi_{2} \cos \theta_{2} \\
\sin \psi_{2} \cos \theta_{2} \\
\sin \theta_{2}
\end{array}\right] \Delta T_{2}=\left[\begin{array}{c}
M_{x_{T}} \\
M_{y_{T}} \\
M_{z_{T}}
\end{array}\right]
\end{aligned}
$$

\subsubsection{Determining the Engine Locations}

Assume the engines are symmetric with respect to the aircraft's XZ-plane. Therefore, the engine's locations become:

$$
\left[\begin{array}{l}
r_{1 x} \\
r_{1 y} \\
r_{1 z}
\end{array}\right]=\left[\begin{array}{c}
r_{2 x} \\
-r_{2 y} \\
r_{2 z}
\end{array}\right]=\left[\begin{array}{l}
r_{x} \\
r_{y} \\
r_{z}
\end{array}\right]
$$

When $\psi_{1}, \theta_{1}, \psi_{2}, \theta_{2}, \Delta T_{1}, \Delta T_{2}$ are known values, as the following notations can be used:

$$
\begin{aligned}
& {\left[\begin{array}{c}
\cos \psi_{1} \cos \theta_{1} \\
\sin \psi_{1} \cos \theta_{1} \\
\sin \theta_{1}
\end{array}\right] \Delta T_{1}=\left[\begin{array}{c}
\varepsilon_{1 x} \\
\varepsilon_{1 y} \\
\varepsilon_{1 z}
\end{array}\right]} \\
& {\left[\begin{array}{c}
\cos \psi_{2} \cos \theta_{2} \\
\sin \psi_{2} \cos \theta_{2} \\
\sin \theta_{2}
\end{array}\right] \Delta T_{2}=\left[\begin{array}{c}
\varepsilon_{2 x} \\
\varepsilon_{2 y} \\
\varepsilon_{2 z}
\end{array}\right]}
\end{aligned}
$$

When equations (6.5), (6.6), and (6.7) are substituted into equation (6.4), it becomes:

$$
\left[\begin{array}{ccc}
0 & \left(\varepsilon_{1 z}-\varepsilon_{2 z}\right) & \left(-\varepsilon_{1 y}-\varepsilon_{2 y}\right) \\
\left(-\varepsilon_{1 z}-\varepsilon_{2 z}\right) & 0 & \left(\varepsilon_{1 x}+\varepsilon_{2 x}\right) \\
\left(\varepsilon_{1 y}-\varepsilon_{2 y}\right) & \left(-\varepsilon_{1 x}+\varepsilon_{2 x}\right) & 0
\end{array}\right]\left[\begin{array}{l}
r_{x} \\
r_{y} \\
r_{z}
\end{array}\right]=\left[\begin{array}{c}
M_{x_{T}} \\
M_{y_{T}} \\
M_{z_{T}}
\end{array}\right]
$$

When $\varepsilon_{1 x}, \varepsilon_{1 y}, \varepsilon_{1 z}, \varepsilon_{2 x}, \varepsilon_{2 y}, \varepsilon_{2 z}, M_{x_{T}}, M_{y_{T}}$, and $M_{z_{T}}$ are known values, then $r_{x}, r_{y}$, and $r_{z}$ can be found if the matrix in (6.8) is invertible, which is equivalent to the corresponding determinant being non-zero: 


$$
\begin{aligned}
& \left|\begin{array}{ccc}
0 & \left(\varepsilon_{1 z}-\varepsilon_{2 z}\right) & \left(-\varepsilon_{1 y}-\varepsilon_{2 y}\right) \\
\left(-\varepsilon_{1 z}-\varepsilon_{2 z}\right) & 0 & \left(\varepsilon_{1 x}+\varepsilon_{2 x}\right) \\
\left(\varepsilon_{1 y}-\varepsilon_{2 y}\right) & \left(-\varepsilon_{1 x}+\varepsilon_{2 x}\right) & 0
\end{array}\right| \neq 0 \\
& -\left(\varepsilon_{1 z}-\varepsilon_{2 z}\right)\left|\begin{array}{cc}
\left(-\varepsilon_{1 z}-\varepsilon_{2 z}\right) & \left(\varepsilon_{1 x}+\varepsilon_{2 x}\right) \\
\left(\varepsilon_{1 y}-\varepsilon_{2 y}\right) & 0
\end{array}\right| \\
& +\left(-\varepsilon_{1 y}-\varepsilon_{2 y}\right)\left|\begin{array}{cc}
\left(-\varepsilon_{1 z}-\varepsilon_{2 z}\right) & 0 \\
\left(\varepsilon_{1 y}-\varepsilon_{2 y}\right) & \left(-\varepsilon_{1 x}+\varepsilon_{2 x}\right)
\end{array}\right| \neq 0 \\
& \left(\varepsilon_{1 z}-\varepsilon_{2 z}\right)\left(\varepsilon_{1 y}-\varepsilon_{2 y}\right)\left(\varepsilon_{1 x}+\varepsilon_{2 x}\right) \\
& +\left(-\varepsilon_{1 y}-\varepsilon_{2 y}\right)\left(-\varepsilon_{1 z}-\varepsilon_{2 z}\right)\left(-\varepsilon_{1 x}+\varepsilon_{2 x}\right) \neq 0 \\
& -\varepsilon_{1 x} \varepsilon_{1 z} \varepsilon_{2 y}-\varepsilon_{1 x} \varepsilon_{1 y} \varepsilon_{2 z}+\varepsilon_{1 y} \varepsilon_{1 z} \varepsilon_{2 x}+\varepsilon_{2 x} \varepsilon_{2 y} \varepsilon_{2 z} \neq 0
\end{aligned}
$$

When (6.6) and (6.7) are substituted back into (6.12) yields:

$$
\begin{aligned}
-\cos \psi_{1} \cos \theta_{1} & \sin \theta_{1} \sin \psi_{2} \cos \theta_{2} \Delta T_{1}^{2} \Delta T_{2} \\
- & \cos \psi_{1} \cos \theta_{1} \cos \theta_{1} \sin \psi_{1} \sin \theta_{2} \Delta T_{1}^{2} \Delta T_{2} \\
& +\sin \psi_{1} \cos \theta_{1} \sin \theta_{1} \cos \psi_{2} \cos \theta_{2} \Delta T_{1}^{2} \Delta T_{2} \\
& +\cos \psi_{2} \sin \psi_{2} \cos \theta_{2} \cos \theta_{2} \sin \theta_{2} \Delta T_{2}^{3} \neq 0
\end{aligned}
$$

When $\Delta T_{1}=\Delta T_{2}=\Delta T$ the $\Delta T$ terms drop out and equation (6.13) becomes:

$$
\begin{gathered}
\sin \theta_{1} \sin \psi_{1} \cos \theta_{1} \cos \psi_{2} \cos \theta_{2}-\sin \theta_{1} \sin \psi_{2} \cos \theta_{2} \cos \psi_{1} \cos \theta_{1} \\
+\sin \theta_{2} \sin \psi_{1} \cos \theta_{1} \cos \psi_{1} \cos \theta_{1} \\
-\sin \theta_{2} \sin \psi_{2} \cos \theta_{2} \cos \psi_{2} \cos \theta_{2} \neq 0
\end{gathered}
$$

If the engine angles are symmetrical with respect the aircraft's CG, then $\theta_{1}=\theta_{2}$ and $\psi_{1}=-\psi_{2}$ and equation (6.14) can be rewritten as: 


$$
\begin{gathered}
\sin \theta_{1} \sin \psi_{1} \cos \theta_{1} \cos \psi_{1} \cos \theta_{1}-\sin \theta_{1}\left(-\sin \psi_{1}\right) \cos \theta_{1} \cos \psi_{1} \cos \theta_{1} \\
+\sin \theta_{1} \sin \psi_{1} \cos \theta_{1} \cos \psi_{1} \cos \theta_{1} \\
-\sin \theta_{1}\left(-\sin \psi_{1}\right) \cos \theta_{1} \cos \psi_{1} \cos \theta_{1} \neq 0 \\
4 \sin \theta_{1} \sin \psi_{1} \cos \theta_{1} \cos \psi_{1} \cos \theta_{1} \neq 0
\end{gathered}
$$

In order for equation (6.16) to remain non-zero, the conditions in Table 6-1 must all be satisfied.

\section{Table 6-1: Conditions for a Non-Zero Determinant for Equation (6.16)}

\begin{tabular}{|c|c|}
\hline Parameter & Condition \\
\hline$\theta_{1}$ & $\neq 0$ \\
\hline$\theta_{1}$ & $\neq \pi / 2$ \\
\hline$\psi_{1}$ & $\neq 0$ \\
\hline$\psi_{1}$ & $\neq \pi / 2$ \\
\hline
\end{tabular}

\subsubsection{Determining the Thrust Control Authority}

Assume the engine locations are known with respect to the aircraft's center of mass.

Furthermore, $\psi_{1}, \theta_{1}, \psi_{2}$, and $\theta_{2}$ are known and established as:

$$
\begin{aligned}
& {\left[\begin{array}{c}
\cos \psi_{1} \cos \theta_{1} \\
\sin \psi_{1} \cos \theta_{1} \\
\sin \theta_{1}
\end{array}\right]=\left[\begin{array}{c}
\varepsilon_{1 x} \\
\varepsilon_{1 y} \\
\varepsilon_{1 z}
\end{array}\right]} \\
& {\left[\begin{array}{c}
\cos \psi_{2} \cos \theta_{2} \\
\sin \psi_{2} \cos \theta_{2} \\
\sin \theta_{2}
\end{array}\right]=\left[\begin{array}{c}
\varepsilon_{2 x} \\
\varepsilon_{2 y} \\
\varepsilon_{2 z}
\end{array}\right]}
\end{aligned}
$$

With these assumptions, $\Delta T_{1}$ and $\Delta T_{2}$ can be found by altering equation (6.4) with equations (6.17) and (6.18) such that: 


$$
\left[\begin{array}{c}
-r_{1 z} \varepsilon_{1 y}+r_{1 y} \varepsilon_{1 z} \\
r_{1 z} \varepsilon_{1 x}-r_{1 x} \varepsilon_{1 z} \\
-r_{1 y} \varepsilon_{1 x}+r_{1 x} \varepsilon_{1 y}
\end{array}\right] \Delta T_{1}+\left[\begin{array}{c}
-r_{2 z} \varepsilon_{2 y}+r_{2 y} \varepsilon_{2 z} \\
r_{2 z} \varepsilon_{2 x}-r_{2 x} \varepsilon_{2 z} \\
-r_{2 y} \varepsilon_{2 x}+r_{2 x} \varepsilon_{2 y}
\end{array}\right] \Delta T_{2}=\left[\begin{array}{c}
M_{x_{T}} \\
M_{y_{T}} \\
M_{z_{T}}
\end{array}\right]
$$

yielding:

$$
\begin{aligned}
& \Delta T_{1} \\
& =\frac{M_{x_{T}}\left(r_{2 z} \varepsilon_{2 x}-r_{2 x} \varepsilon_{2 z}\right)+M_{y_{T}}\left(r_{2 z} \varepsilon_{2 y}-r_{2 y} \varepsilon_{2 z}\right)}{\left(r_{1 z} \varepsilon_{1 x}-r_{1 z} \varepsilon_{1 z}\right)\left(r_{2 z} \varepsilon_{2 y}-r_{2 y} \varepsilon_{2 z}\right)+\left(r_{1 y} \varepsilon_{1 z}-r_{1 z} \varepsilon_{1 y}\right)\left(r_{2 z} \varepsilon_{2 x}-r_{2 x} \varepsilon_{2 z}\right)} \\
& \Delta T_{2} \\
& =\frac{M_{x_{T}}\left(r_{1 z} \varepsilon_{1 z}-r_{1 z} \varepsilon_{1 x}\right)+M_{y_{T}}\left(r_{1 y} \varepsilon_{1 y}-r_{1 z} \varepsilon_{1 y}\right)}{\left(r_{1 z} \varepsilon_{1 x}-r_{1 z} \varepsilon_{1 z}\right)\left(r_{2 z} \varepsilon_{2 y}-r_{2 y} \varepsilon_{2 z}\right)+\left(r_{1 y} \varepsilon_{1 z}-r_{1 z} \varepsilon_{1 y}\right)\left(r_{2 z} \varepsilon_{2 x}-r_{2 x} \varepsilon_{2 z}\right)}
\end{aligned}
$$

A solution exists as long as the third equation in (6.19) is equal to the first equation multiplied by a constant, $\gamma$, equal to:

$$
\gamma=\frac{M_{x_{T}}}{M_{z_{T}}}
$$

\subsubsection{Determining the Engine Euler Angles}

Assume the engine locations are known with respect to the aircraft's center of mass.

Furthermore, $\Delta T_{1}$ and $\Delta T_{2}$ are known values. Then the following applies:

$$
\left[\begin{array}{ccc}
0 & -r_{1 z} & r_{1 y} \\
r_{1 z} & 0 & -r_{1 x} \\
-r_{1 y} & r_{1 x} & 0
\end{array}\right] \Delta T_{1}=\left[\begin{array}{ccc}
0 & -r_{1 z} \Delta T_{1} & r_{1 y} \Delta T_{1} \\
r_{1 z} \Delta T_{1} & 0 & -r_{1 x} \Delta T_{1} \\
-r_{1 y} \Delta T_{1} & r_{1 x} \Delta T_{1} & 0
\end{array}\right]
$$




$$
\left[\begin{array}{ccc}
0 & -r_{2 z} & r_{2 y} \\
r_{2 z} & 0 & -r_{2 x} \\
-r_{2 y} & r_{2 x} & 0
\end{array}\right] \Delta T_{2}=\left[\begin{array}{ccc}
0 & -r_{2 z} \Delta T_{2} & r_{2 y} \Delta T_{2} \\
r_{2 z} \Delta T_{2} & 0 & -r_{2 x} \Delta T_{2} \\
-r_{2 y} \Delta T_{2} & r_{2 x} \Delta T_{2} & 0
\end{array}\right]
$$

Equations (6.23) and (6.24) can be substituted into equation (6.4) such that:

$$
\begin{gathered}
{\left[\begin{array}{ccc}
0 & -r_{1 z} \Delta T_{1} & r_{1 y} \Delta T_{1} \\
r_{1 z} \Delta T_{1} & 0 & -r_{1 x} \Delta T_{1} \\
-r_{1 y} \Delta T_{1} & r_{1 x} \Delta T_{1} & 0
\end{array}\right]\left[\begin{array}{c}
\cos \psi_{1} \cos \theta_{1} \\
\sin \psi_{1} \cos \theta_{1} \\
\sin \theta_{1}
\end{array}\right]} \\
+\left[\begin{array}{ccc}
0 & -r_{2 z} \Delta T_{2} & r_{2 y} \Delta T_{2} \\
r_{2 z} \Delta T_{2} & 0 & -r_{2 x} \Delta T_{2} \\
-r_{2 y} \Delta T_{2} & r_{2 x} \Delta T_{2} & 0
\end{array}\right]\left[\begin{array}{c}
\cos \psi_{2} \cos \theta_{2} \\
\sin \psi_{2} \cos \theta_{2} \\
\sin \theta_{2}
\end{array}\right]=\left[\begin{array}{c}
M_{x_{T}} \\
M_{y_{T}} \\
M_{z_{T}}
\end{array}\right]
\end{gathered}
$$

This leaves four unknowns and three equations. Set:

$$
\theta_{1}=\theta_{2}
$$

When equation (6.26) is used to simplify equation (6.25), the equation becomes:

$$
\begin{gathered}
{\left[\begin{array}{ccc}
0 & -r_{1 z} \Delta T_{1} & r_{1 y} \Delta T_{1} \\
r_{1 z} \Delta T_{1} & 0 & -r_{1 x} \Delta T_{1} \\
-r_{1 y} \Delta T_{1} & r_{1 x} \Delta T_{1} & 0
\end{array}\right]\left[\begin{array}{c}
\cos \psi_{1} \cos \theta_{1} \\
\sin \psi_{1} \cos \theta_{1} \\
\sin \theta_{1}
\end{array}\right]} \\
+\left[\begin{array}{ccc}
0 & -r_{2 z} \Delta T_{2} & r_{2 y} \Delta T_{2} \\
r_{2 z} \Delta T_{2} & 0 & -r_{2 x} \Delta T_{2} \\
-r_{2 y} \Delta T_{2} & r_{2 x} \Delta T_{2} & 0
\end{array}\right]\left[\begin{array}{c}
\cos \psi_{2} \cos \theta_{1} \\
\sin \psi_{2} \cos \theta_{1} \\
\sin \theta_{1}
\end{array}\right]=\left[\begin{array}{c}
M_{x_{T}} \\
M_{y_{T}} \\
M_{z_{T}}
\end{array}\right]
\end{gathered}
$$

Now $\psi_{1}, \theta_{1}$, and $\psi_{2}$ can be found numerically.

If equation (6.26) instead was set such that:

$$
\psi_{1}=-\psi_{2}
$$

Then using equation (6.28), equation (6.25) becomes 


$$
\begin{gathered}
{\left[\begin{array}{ccc}
0 & -r_{1 z} \Delta T_{1} & r_{1 y} \Delta T_{1} \\
r_{1 z} \Delta T_{1} & 0 & -r_{1 x} \Delta T_{1} \\
-r_{1 y} \Delta T_{1} & r_{1 x} \Delta T_{1} & 0
\end{array}\right]\left[\begin{array}{c}
\cos \psi_{1} \cos \theta_{1} \\
\sin \psi_{1} \cos \theta_{1} \\
\sin \theta_{1}
\end{array}\right]} \\
+\left[\begin{array}{ccc}
0 & -r_{2 z} \Delta T_{2} & r_{2 y} \Delta T_{2} \\
r_{2 z} \Delta T_{2} & 0 & -r_{2 x} \Delta T_{2} \\
-r_{2 y} \Delta T_{2} & r_{2 x} \Delta T_{2} & 0
\end{array}\right]\left[\begin{array}{c}
-\cos \psi_{1} \cos \theta_{2} \\
\sin \psi_{1} \cos \theta_{2} \\
\sin \theta_{2}
\end{array}\right] \\
=\left[\begin{array}{c}
M_{x_{T}} \\
M_{y_{T}} \\
M_{z_{T}}
\end{array}\right]
\end{gathered}
$$

Now $\psi_{1}, \theta_{1}$, and $\theta_{2}$ can be found numerically.

\subsection{Odd Engine Number Model Example}

When $\mathrm{n}$ is equal to three, equation (6.3) becomes:

$$
\begin{aligned}
{\left[\begin{array}{ccc}
0 & -r_{1 z} & r_{1 y} \\
r_{1 z} & 0 & -r_{1 x} \\
-r_{1 y} & r_{1 x} & 0
\end{array}\right]\left[\begin{array}{c}
\cos \psi_{1} \cos \theta_{1} \\
\sin \psi_{1} \cos \theta_{1} \\
\sin \theta_{1}
\end{array}\right] \Delta T_{1} } \\
+\left[\begin{array}{ccc}
0 & -r_{2 z} & r_{2 y} \\
r_{2 z} & 0 & -r_{2 x} \\
-r_{2 y} & r_{2 x} & 0
\end{array}\right]\left[\begin{array}{c}
\cos \psi_{2} \cos \theta_{2} \\
\sin \psi_{2} \cos \theta_{2} \\
\sin \theta_{2}
\end{array}\right] \Delta T_{2} \\
+\left[\begin{array}{ccc}
0 & -r_{3 z} & r_{3 y} \\
r_{3 z} & 0 & -r_{3 x} \\
-r_{3 y} & r_{3 x} & 0
\end{array}\right]\left[\begin{array}{c}
\cos \psi_{3} \cos \theta_{3} \\
\sin \psi_{3} \cos \theta_{3} \\
\sin \theta_{3}
\end{array}\right] \Delta T_{3}=\left[\begin{array}{c}
M_{x_{T}} \\
M_{y_{T}} \\
M_{z_{T}}
\end{array}\right]
\end{aligned}
$$

Assuming that the $\mathrm{n}=2$ engine is located along the $\mathrm{XZ}$-plane. Equation (6.30) becomes: 


$$
\begin{gathered}
{\left[\begin{array}{ccc}
0 & -r_{1 z} & r_{1 y} \\
r_{1 z} & 0 & -r_{1 x} \\
-r_{1 y} & r_{1 x} & 0
\end{array}\right]\left[\begin{array}{c}
\cos \psi_{1} \cos \theta_{1} \\
\sin \psi_{1} \cos \theta_{1} \\
\sin \theta_{1}
\end{array}\right] \Delta T_{1}} \\
+\left[\begin{array}{ccc}
0 & -r_{2 z} & 0 \\
r_{2 z} & 0 & -r_{2 x} \\
0 & r_{2 x} & 0
\end{array}\right]\left[\begin{array}{c}
\cos \psi_{2} \cos \theta_{2} \\
\sin \psi_{2} \cos \theta_{2} \\
\sin \theta_{2}
\end{array}\right] \Delta T_{2} \\
+\left[\begin{array}{ccc}
0 & -r_{3 z} & r_{3 y} \\
r_{3 z} & 0 & -r_{3 x} \\
-r_{3 y} & r_{3 x} & 0
\end{array}\right]\left[\begin{array}{c}
\cos \psi_{3} \cos \theta_{3} \\
\sin \psi_{3} \cos \theta_{3} \\
\sin \theta_{3}
\end{array}\right] \Delta T_{3}=\left[\begin{array}{c}
M_{x_{T}} \\
M_{y_{T}} \\
M_{z_{T}}
\end{array}\right]
\end{gathered}
$$

\subsubsection{Determining the Engine Locations}

Assume the $n=1$ and $n=3$ engine locations, Euler angles, and differential thrust values are fully known. Then, equation (6.31) can be simplified using

$$
\begin{aligned}
& {\left[\begin{array}{ccc}
0 & -r_{1 z} & r_{1 y} \\
r_{1 z} & 0 & -r_{1 x} \\
-r_{1 y} & r_{1 x} & 0
\end{array}\right]\left[\begin{array}{c}
\cos \psi_{1} \cos \theta_{1} \\
\sin \psi_{1} \cos \theta_{1} \\
\sin \theta_{1}
\end{array}\right] \Delta T_{1}} \\
& =\left[\begin{array}{c}
-r_{1 z} \sin \psi_{1} \cos \theta_{1}+r_{1 y} \sin \theta_{1} \\
r_{1 z} \cos \psi_{1} \cos \theta_{1}-r_{1 x} \sin \theta_{1} \\
-r_{1 y} \cos \psi_{1} \cos \theta_{1}+r_{1 x} \sin \psi_{1} \cos \theta_{1}
\end{array}\right] \Delta T_{1}=\left[\begin{array}{c}
\varepsilon_{1 x} \\
\varepsilon_{1 y} \\
\varepsilon_{1 z}
\end{array}\right] \Delta T_{1} \\
& {\left[\begin{array}{c}
\cos \psi_{2} \cos \theta_{2} \\
\sin \psi_{2} \cos \theta_{2} \\
\sin \theta_{2}
\end{array}\right] \Delta T_{2}=\left[\begin{array}{c}
\varepsilon_{2 x} \\
\varepsilon_{2 y} \\
\varepsilon_{2 z}
\end{array}\right] \Delta T_{2}} \\
& {\left[\begin{array}{ccc}
0 & -r_{3 z} & r_{3 y} \\
r_{3 z} & 0 & -r_{3 x} \\
-r_{3 y} & r_{3 x} & 0
\end{array}\right]\left[\begin{array}{c}
\cos \psi_{3} \cos \theta_{3} \\
\sin \psi_{3} \cos \theta_{3} \\
\sin \theta_{3}
\end{array}\right] \Delta T_{3}} \\
& =\left[\begin{array}{c}
-r_{3 z} \sin \psi_{3} \cos \theta_{1}+r_{3 y} \sin \theta_{3} \\
r_{3 z} \cos \psi_{3} \cos \theta_{3}-r_{3 x} \sin \theta_{3} \\
-r_{3 y} \cos \psi_{3} \cos \theta_{3}+r_{3 x} \sin \psi_{3} \cos \theta_{3}
\end{array}\right] \Delta T_{3}=\left[\begin{array}{c}
\varepsilon_{3 x} \\
\varepsilon_{3 y} \\
\varepsilon_{3 z}
\end{array}\right] \Delta T_{3}
\end{aligned}
$$

to become 


$$
\left[\begin{array}{c}
\varepsilon_{1 x} \\
\varepsilon_{1 y} \\
\varepsilon_{1 z}
\end{array}\right] \Delta T_{1}+\left[\begin{array}{ccc}
0 & -r_{2 z} & 0 \\
r_{2 z} & 0 & -r_{2 x} \\
0 & r_{2 x} & 0
\end{array}\right]\left[\begin{array}{c}
\varepsilon_{2 x} \\
\varepsilon_{2 y} \\
\varepsilon_{2 z}
\end{array}\right] \Delta T_{2}+\left[\begin{array}{c}
\varepsilon_{3 x} \\
\varepsilon_{3 y} \\
\varepsilon_{3 z}
\end{array}\right] \Delta T_{3}=\left[\begin{array}{c}
M_{x_{T}} \\
M_{y_{T}} \\
M_{z_{T}}
\end{array}\right]
$$

Equation (6.31) can be rewritten as

$$
\left[\begin{array}{ccc}
0 & -r_{2 z} & 0 \\
r_{2 z} & 0 & -r_{2 x} \\
0 & r_{2 x} & 0
\end{array}\right]\left[\begin{array}{c}
\varepsilon_{2 x} \\
\varepsilon_{2 y} \\
\varepsilon_{2 z}
\end{array}\right] \Delta T_{2}+\left[\begin{array}{c}
\varepsilon_{1 x} \Delta T_{1}+\varepsilon_{3 x} \Delta T_{3} \\
\varepsilon_{1 y} \Delta T_{1}+\varepsilon_{3 y} \Delta T_{3} \\
\varepsilon_{1 z} \Delta T_{1}+\varepsilon_{3 z} \Delta T_{3}
\end{array}\right]=\left[\begin{array}{c}
M_{x_{T}} \\
M_{y_{T}} \\
M_{z_{T}}
\end{array}\right]
$$

From equation (6.36) $r_{2 x}, r_{2 z}$, and $\Delta T_{2}$ can be determined such that:

$$
\begin{gathered}
r_{2 x}=\frac{M_{z_{T}}-\varepsilon_{1 z} \Delta T_{1}-\varepsilon_{3 z} \Delta T_{3}}{\varepsilon_{2 y} \Delta T_{2}} \\
r_{2 z}=\frac{\varepsilon_{1 x} \Delta T_{1}+\varepsilon_{3 z} \Delta T_{3}-M_{x_{T}}}{\varepsilon_{2 y} \Delta T_{2}} \\
\Delta T_{2}=\frac{M_{y_{T}}-\varepsilon_{1 y} \Delta T_{1}-\varepsilon_{3 y} \Delta T_{3}}{r_{2 z} \varepsilon_{2 x}-r_{2 x} \varepsilon_{2 z}}
\end{gathered}
$$

Equations (6.37), (6.38), and (6.39) can now be solved numerically.

If $n=2$ engine location, Euler angles, and differential thrust values are fully known and the $n=1$ engine and $n=3$ engine are symmetric such that:

$$
\left[\begin{array}{lll}
r_{1 x} & r_{1 y} & r_{1 z}
\end{array}\right]=\left[\begin{array}{lll}
r_{3 x} & -r_{3 y} & r_{3 z}
\end{array}\right]
$$

then equation (6.31) can be simplified using

$$
\left[\begin{array}{c}
\cos \psi_{1} \cos \theta_{1} \\
\sin \psi_{1} \cos \theta_{1} \\
\sin \theta_{1}
\end{array}\right] \Delta T_{1}=\left[\begin{array}{c}
\varepsilon_{1 x} \\
\varepsilon_{1 y} \\
\varepsilon_{1 z}
\end{array}\right] \Delta T_{1}
$$




$$
\begin{aligned}
& {\left[\begin{array}{ccc}
0 & -r_{2 z} & 0 \\
r_{2 z} & 0 & -r_{2 x} \\
0 & r_{2 x} & 0
\end{array}\right]\left[\begin{array}{c}
\cos \psi_{2} \cos \theta_{2} \\
\sin \psi_{2} \cos \theta_{2} \\
\sin \theta_{2}
\end{array}\right] \Delta T_{2} } \\
&=\left[\begin{array}{c}
-r_{2 z} \sin \psi_{2} \cos \theta_{2} \\
r_{2 z} \cos \psi_{2} \cos \theta_{2}-r_{2 x} \sin \theta_{2} \\
r_{2 x} \sin \psi_{2} \cos \theta_{2}
\end{array}\right] \Delta T_{2}=\left[\begin{array}{c}
\varepsilon_{2 x} \\
\varepsilon_{2 y} \\
\varepsilon_{2 z}
\end{array}\right] \Delta T_{2} \\
& {\left[\begin{array}{c}
\cos \psi_{3} \cos \theta_{3} \\
\sin \psi_{3} \cos \theta_{3} \\
\sin \theta_{3}
\end{array}\right] \Delta T_{3}=\left[\begin{array}{c}
\varepsilon_{3 x} \\
\varepsilon_{3 y} \\
\varepsilon_{3 z}
\end{array}\right] \Delta T_{3} }
\end{aligned}
$$

to become:

$$
\begin{gathered}
{\left[\begin{array}{ccc}
0 & -r_{1 z} & r_{1 y} \\
r_{1 z} & 0 & -r_{1 x} \\
-r_{1 y} & r_{1 x} & 0
\end{array}\right]\left[\begin{array}{l}
\varepsilon_{1 x} \\
\varepsilon_{1 y} \\
\varepsilon_{1 z}
\end{array}\right] \Delta T_{1}+\left[\begin{array}{l}
\varepsilon_{2 x} \\
\varepsilon_{2 y} \\
\varepsilon_{2 z}
\end{array}\right] \Delta T_{2}+\left[\begin{array}{ccc}
0 & -r_{1 z} & -r_{1 y} \\
r_{1 z} & 0 & -r_{1 x} \\
r_{1 y} & r_{1 x} & 0
\end{array}\right]\left[\begin{array}{l}
\varepsilon_{3 x} \\
\varepsilon_{3 y} \\
\varepsilon_{3 z}
\end{array}\right] \Delta T_{3}} \\
=\left[\begin{array}{l}
M_{x_{T}} \\
M_{y_{T}} \\
M_{z_{T}}
\end{array}\right]
\end{gathered}
$$

or:

$$
\begin{gathered}
{\left[\begin{array}{ccc}
0 & \varepsilon_{1 z} \Delta T_{1}-\varepsilon_{3 z} \Delta T_{3} & -\left(\varepsilon_{1 y} \Delta T_{1}+\varepsilon_{3 y} \Delta T_{3}\right) \\
-\left(\varepsilon_{1 z} \Delta T_{1}+\varepsilon_{3 z} \Delta T_{3}\right) & 0 & \varepsilon_{1 x} \Delta T_{1}+\varepsilon_{3 x} \Delta T_{3} \\
\varepsilon_{1 y} \Delta T_{1}+\varepsilon_{3 y} \Delta T_{3} & -\left(\varepsilon_{1 x} \Delta T_{1}-\varepsilon_{3 x} \Delta T_{3}\right) & 0
\end{array}\right]\left[\begin{array}{l}
r_{1 x} \\
r_{1 y} \\
r_{1 z}
\end{array}\right]} \\
=\left[\begin{array}{l}
M_{x_{T}}-\varepsilon_{2 x} \Delta T_{2} \\
M_{y_{T}}-\varepsilon_{2 y} \Delta T_{2} \\
M_{z_{T}}-\varepsilon_{2 z} \Delta T_{2}
\end{array}\right]
\end{gathered}
$$

From equation (6.45) $r_{1 x}, r_{1 y}$, and $r_{1 z}$ can be determined if the determinant in the matrix is nonzero. The determinant yields:

$$
\begin{gathered}
\varepsilon_{1 y} \varepsilon_{1 z} \varepsilon_{3 x} \Delta T_{1}^{2} \Delta T_{3}-\varepsilon_{1 x} \varepsilon_{1 y} \varepsilon_{3 z} \Delta T_{1}^{2} \Delta T_{3}-\varepsilon_{1 x} \varepsilon_{3 y} \varepsilon_{3 z} \Delta T_{1} \Delta T_{3}^{2} \\
-\varepsilon_{1 z} \varepsilon_{3 x} \varepsilon_{3 y} \Delta T_{1} \Delta T_{3}^{2} \neq 0
\end{gathered}
$$


When equations (6.41) and (6.43) are substituted back into equation (6.46) it becomes:

$\sin \psi_{1} \cos \theta_{1} \sin \theta_{1} \cos \psi_{3} \cos \theta_{3} \Delta T_{1}^{2} \Delta T_{3}$

$$
\begin{aligned}
& -\cos \psi_{1} \cos \theta_{1} \sin \psi_{1} \cos \theta_{1} \sin \theta_{3} \Delta T_{1}^{2} \Delta T_{3} \\
& -\cos \psi_{1} \cos \theta_{1} \sin \psi_{3} \cos \theta_{3} \sin \theta_{3} \Delta T_{1} \Delta T_{3}^{2} \\
& -\sin \theta_{1} \cos \psi_{3} \cos \theta_{3} \sin \psi_{3} \cos \theta_{3} \Delta T_{1} \Delta T_{3}^{2} \neq 0
\end{aligned}
$$

When $\Delta T_{1}=\Delta T_{3}=\Delta T$ the $\Delta T$ terms drop out and equation (6.47) becomes:

$$
\begin{gathered}
\sin \psi_{1} \cos \theta_{1} \sin \theta_{1} \cos \psi_{3} \cos \theta_{3}-\cos \psi_{1} \cos \theta_{1} \sin \psi_{1} \cos \theta_{1} \sin \theta_{3} \\
-\cos \psi_{1} \cos \theta_{1} \sin \psi_{3} \cos \theta_{3} \sin \theta_{3} \\
-\sin \theta_{1} \cos \psi_{3} \cos \theta_{3} \sin \psi_{3} \cos \theta_{3} \neq 0
\end{gathered}
$$

If the engine angles are symmetrical with respect the aircraft's CG, then $\theta_{1}=\theta_{3}$ and $\psi_{1}=-\psi_{3}$ and equation (6.48) can be rewritten as:

$$
\begin{gathered}
\sin \theta_{1} \sin \psi_{1} \cos \theta_{1} \cos \psi_{1} \cos \theta_{1}-\sin \theta_{1}\left(-\sin \psi_{1}\right) \cos \theta_{1} \cos \psi_{1} \cos \theta_{1} \\
+\sin \theta_{1} \sin \psi_{1} \cos \theta_{1} \cos \psi_{1} \cos \theta_{1} \\
-\sin \theta_{1}\left(-\sin \psi_{1}\right) \cos \theta_{1} \cos \psi_{1} \cos \theta_{1} \neq 0 \\
4 \sin \theta_{1} \sin \psi_{1} \cos \theta_{1} \cos \psi_{1} \cos \theta_{1} \neq 0
\end{gathered}
$$

To ensure a non-zero determinate, the conditions in Table 6-2 must be satisfied.

Table 6-2: Conditions for a Non-Zero determinate for Equation (6.50)

\begin{tabular}{|c|c|}
\hline Parameter & Condition \\
\hline$\theta_{1}$ & $\neq 0$ \\
\hline$\theta_{1}$ & $\neq \pi / 2$ \\
\hline$\psi_{1}$ & $\neq 0$ \\
\hline$\psi_{1}$ & $\neq \pi / 2$ \\
\hline
\end{tabular}




\subsubsection{Determining the Thrust Control Authority}

If the Euler angles and engine locations are fully known, then equation (6.31) can be simplified using:

$$
\begin{aligned}
& {\left[\begin{array}{ccc}
0 & -r_{1 z} & r_{1 y} \\
r_{1 z} & 0 & -r_{1 x} \\
-r_{1 y} & r_{1 x} & 0
\end{array}\right]\left[\begin{array}{c}
\cos \psi_{1} \cos \theta_{1} \\
\sin \psi_{1} \cos \theta_{1} \\
\sin \theta_{1}
\end{array}\right]} \\
& =\left[\begin{array}{c}
-r_{1 z} \sin \psi_{1} \cos \theta_{1}+r_{1 y} \sin \theta_{1} \\
r_{1 z} \cos \psi_{1} \cos \theta_{1}-r_{1 x} \sin \theta_{1} \\
-r_{1 y} \cos \psi_{1} \cos \theta_{1}+r_{1 x} \sin \psi_{1} \cos \theta_{1}
\end{array}\right]=\left[\begin{array}{l}
\varepsilon_{1 x} \\
\varepsilon_{1 y} \\
\varepsilon_{1 z}
\end{array}\right] \\
& {\left[\begin{array}{ccc}
0 & -r_{2 z} & 0 \\
r_{2 z} & 0 & -r_{2 x} \\
0 & r_{2 x} & 0
\end{array}\right]\left[\begin{array}{c}
\cos \psi_{2} \cos \theta_{2} \\
\sin \psi_{2} \cos \theta_{2} \\
\sin \theta_{2}
\end{array}\right]=\left[\begin{array}{c}
-r_{2 z} \sin \psi_{2} \cos \theta_{1} \\
r_{2 z} \cos \psi_{2} \cos \theta_{2}-r_{2 x} \sin \theta_{2} \\
r_{2 x} \sin \psi_{2} \cos \theta_{2}
\end{array}\right]} \\
& {\left[\begin{array}{ccc}
0 & -r_{3 z} & r_{3 y} \\
r_{3 z} & 0 & -r_{3 x} \\
-r_{3 y} & r_{3 x} & 0
\end{array}\right]\left[\begin{array}{c}
\cos \psi_{3} \cos \theta_{3} \\
\sin \psi_{3} \cos \theta_{3} \\
\sin \theta_{3}
\end{array}\right]} \\
& \left.\left.\begin{array}{c}
\varepsilon_{2 y} \\
\varepsilon_{2 z}
\end{array}\right] \begin{array}{c}
-r_{3 z} \sin \psi_{3} \cos \theta_{3}+r_{3 y} \sin \theta_{3} \\
r_{1 z} \cos \psi_{3} \cos \theta_{3}-r_{3 x} \sin \theta_{3} \\
-r_{3 y} \cos \psi_{3} \cos \theta_{3}+r_{3 x} \sin \psi_{3} \cos \theta_{3}
\end{array}\right]=\left[\begin{array}{c}
\varepsilon_{3 x} \\
\varepsilon_{3 y} \\
\varepsilon_{3 z}
\end{array}\right]
\end{aligned}
$$

to become:

$$
\left[\begin{array}{c}
\varepsilon_{1 x} \\
\varepsilon_{1 y} \\
\varepsilon_{1 z}
\end{array}\right] \Delta T_{1}+\left[\begin{array}{l}
\varepsilon_{2 x} \\
\varepsilon_{2 y} \\
\varepsilon_{2 z}
\end{array}\right] \Delta T_{2}+\left[\begin{array}{l}
\varepsilon_{3 x} \\
\varepsilon_{3 y} \\
\varepsilon_{3 z}
\end{array}\right] \Delta T_{3}=\left[\begin{array}{l}
M_{x_{T}} \\
M_{y_{T}} \\
M_{z_{T}}
\end{array}\right]
$$

or: 


$$
\left[\begin{array}{ccc}
\varepsilon_{1 x} & \varepsilon_{2 x} & \varepsilon_{3 x} \\
\varepsilon_{1 y} & \varepsilon_{2 y} & \varepsilon_{3 y} \\
\varepsilon_{1 z} & \varepsilon_{2 z} & \varepsilon_{3 z}
\end{array}\right]\left[\begin{array}{l}
\Delta T_{1} \\
\Delta T_{2} \\
\Delta T_{3}
\end{array}\right]=\left[\begin{array}{c}
M_{x_{T}} \\
M_{y_{T}} \\
M_{z_{T}}
\end{array}\right]
$$

For equation (6.55) to have a solution, the determinate of the matrix must be non-zero. Following the methodology in previous sections, the determinant is determined as:

$$
\varepsilon_{1 x}\left(\varepsilon_{2 y} \varepsilon_{3 z}-\varepsilon_{2 z} \varepsilon_{3 y}\right)-\varepsilon_{2 x}\left(\varepsilon_{1 y} \varepsilon_{3 z}-\varepsilon_{1 z} \varepsilon_{3 y}\right)+\varepsilon_{3 x}\left(\varepsilon_{1 y} \varepsilon_{2 z}-\varepsilon_{1 z} \varepsilon_{2 y}\right) \neq 0
$$

When equations (6.51), (6.52), and (6.53) are substituted into equation (6.56), several conditions need to be satisfied in order for the equation to remain non-zero. These are outlined in Table 6-3.

Table 6-3: Conditions for a Non-Zero for Equation (6.56)

\begin{tabular}{|c|c|}
\hline Parameter & Condition \\
\hline$\theta_{1}$ & $\neq 0$ \\
\hline$\theta_{1}$ & $\neq \pi / 2$ \\
\hline$\psi_{1}$ & $\neq 0$ \\
\hline$\psi_{1}$ & $\neq \pi / 2$ \\
\hline$\theta_{2}$ & $\neq 0$ \\
\hline$\theta_{2}$ & $\neq \pi / 2$ \\
\hline$\psi_{2}$ & $\neq 0$ \\
\hline$\psi_{2}$ & $\neq \pi / 2$ \\
\hline$\theta_{3}$ & $\neq 0$ \\
\hline$\theta_{3}$ & $\neq \pi / 2$ \\
\hline$\psi_{3}$ & $\neq 0$ \\
\hline$\psi_{3}$ & $\neq \pi / 2$ \\
\hline
\end{tabular}

\subsubsection{Determining the Engine Euler Angles}

If the engine locations and differential thrust are known, the Euler angles can be found by simplifying equation (6.31) using: 


$$
\begin{gathered}
{\left[\begin{array}{ccc}
0 & -r_{1 z} & r_{1 y} \\
r_{1 z} & 0 & -r_{1 x} \\
-r_{1 y} & r_{1 x} & 0
\end{array}\right] \Delta T_{1}=\left[\begin{array}{c}
-r_{1 z} \Delta T_{1}+r_{1 y} \Delta T_{1} \\
r_{1 z} \Delta T_{1}-r_{1 x} \Delta T_{1} \\
-r_{1 y} \Delta T_{1}+r_{1 x} \Delta T_{1}
\end{array}\right]=\left[\begin{array}{c}
\varepsilon_{1 x} \\
\varepsilon_{1 y} \\
\varepsilon_{1 z}
\end{array}\right]} \\
{\left[\begin{array}{ccc}
0 & -r_{2 z} & 0 \\
r_{2 z} & 0 & -r_{2 x} \\
0 & r_{2 x} & 0
\end{array}\right] \Delta T_{2}=\left[\begin{array}{c}
-r_{2 z} \Delta T_{2} \\
r_{2 z} \Delta T_{2}-r_{2 x} \Delta T_{2} \\
r_{2 x} \Delta T_{2}
\end{array}\right]=\left[\begin{array}{c}
\varepsilon_{2 x} \\
\varepsilon_{2 y} \\
\varepsilon_{2 z}
\end{array}\right]} \\
{\left[\begin{array}{ccc}
0 & -r_{3 z} & r_{3 y} \\
r_{3 z} & 0 & -r_{3 x} \\
-r_{3 y} & r_{3 x} & 0
\end{array}\right] \Delta T_{3}=\left[\begin{array}{c}
-r_{3 z} \Delta T_{3}+r_{3 y} \Delta T_{3} \\
r_{3 z} \Delta T_{3}-r_{3 x} \Delta T_{3} \\
-r_{3 y} \Delta T_{3}+r_{3 x} \Delta T_{3}
\end{array}\right]=\left[\begin{array}{c}
\varepsilon_{3 x} \\
\varepsilon_{3 y} \\
\varepsilon_{3 z}
\end{array}\right]}
\end{gathered}
$$

to become:

$$
\begin{gathered}
{\left[\begin{array}{c}
\varepsilon_{1 x} \\
\varepsilon_{1 y} \\
\varepsilon_{1 z}
\end{array}\right]\left[\begin{array}{c}
\cos \psi_{1} \cos \theta_{1} \\
\sin \psi_{1} \cos \theta_{1} \\
\sin \theta_{1}
\end{array}\right]+\left[\begin{array}{c}
\varepsilon_{2 x} \\
\varepsilon_{2 y} \\
\varepsilon_{2 z}
\end{array}\right]\left[\begin{array}{c}
\cos \psi_{2} \cos \theta_{2} \\
\sin \psi_{2} \cos \theta_{2} \\
\sin \theta_{2}
\end{array}\right]+\left[\begin{array}{c}
\varepsilon_{3 x} \\
\varepsilon_{3 y} \\
\varepsilon_{3 z}
\end{array}\right]\left[\begin{array}{c}
\cos \psi_{3} \cos \theta_{3} \\
\sin \psi_{3} \cos \theta_{3} \\
\sin \theta_{3}
\end{array}\right]} \\
=\left[\begin{array}{c}
M_{x_{T}} \\
M_{y_{T}} \\
M_{z_{T}}
\end{array}\right]
\end{gathered}
$$

If the $\theta$ components are held constant, equation (6.60) can be simplified by rewriting equations (6.57), (6.58), and (6.59) such that:

$$
\begin{gathered}
{\left[\begin{array}{ccc}
0 & -r_{1 z} & r_{1 y} \\
r_{1 z} & 0 & -r_{1 x} \\
-r_{1 y} & r_{1 x} & 0
\end{array}\right]\left[\begin{array}{c}
\cos \theta_{1} \\
\cos \theta_{1} \\
\sin \theta_{1}
\end{array}\right] \Delta T_{1}=\left[\begin{array}{c}
-r_{1 z} \Delta T_{1}+r_{1 y} \Delta T_{1} \\
r_{1 z} \Delta T_{1}-r_{1 x} \Delta T_{1} \\
-r_{1 y} \Delta T_{1}+r_{1 x} \Delta T_{1}
\end{array}\right]\left[\begin{array}{l}
\cos \theta_{1} \\
\cos \theta_{1} \\
\sin \theta_{1}
\end{array}\right]=\left[\begin{array}{l}
\varepsilon_{1 x} \\
\varepsilon_{1 y} \\
\varepsilon_{1 z}
\end{array}\right]} \\
{\left[\begin{array}{ccc}
0 & -r_{2 z} & 0 \\
r_{2 z} & 0 & -r_{2 x} \\
0 & r_{2 x} & 0
\end{array}\right]\left[\begin{array}{c}
\cos \theta_{2} \\
\cos \theta_{2} \\
\sin \theta_{2}
\end{array}\right] \Delta T_{2}=\left[\begin{array}{c}
-r_{2 z} \Delta T_{2} \\
r_{2 z} \Delta T_{2}-r_{2 x} \Delta T_{2} \\
r_{2 x} \Delta T_{2}
\end{array}\right]\left[\begin{array}{c}
\cos \theta_{2} \\
\cos \theta_{2} \\
\sin \theta_{2}
\end{array}\right]=\left[\begin{array}{c}
\varepsilon_{2 x} \\
\varepsilon_{2 y} \\
\varepsilon_{2 z}
\end{array}\right]} \\
{\left[\begin{array}{ccc}
0 & -r_{3 z} & r_{3 y} \\
r_{3 z} & 0 & -r_{3 x} \\
-r_{3 y} & r_{3 x} & 0
\end{array}\right]\left[\begin{array}{c}
\cos \theta_{3} \\
\cos \theta_{3} \\
\sin \theta_{3}
\end{array}\right] \Delta T_{3}=\left[\begin{array}{c}
-r_{3 z} \Delta T_{3}+r_{3 y} \Delta T_{3} \\
r_{3 z} \Delta T_{3}-r_{3 x} \Delta T_{3} \\
-r_{3 y} \Delta T_{3}+r_{3 x} \Delta T_{3}
\end{array}\right]\left[\begin{array}{l}
\cos \theta_{3} \\
\cos \theta_{3} \\
\sin \theta_{3}
\end{array}\right]=\left[\begin{array}{c}
\varepsilon_{3 x} \\
\varepsilon_{3 y} \\
\varepsilon_{3 z}
\end{array}\right]}
\end{gathered}
$$

yielding: 


$$
\left[\begin{array}{c}
\varepsilon_{1 x} \cos \psi_{1} \\
\varepsilon_{1 y} \sin \psi_{1} \\
\varepsilon_{1 z}
\end{array}\right]+\left[\begin{array}{c}
\varepsilon_{2 x} \cos \psi_{2} \\
\varepsilon_{2 y} \sin \psi_{2} \\
\varepsilon_{2 z}
\end{array}\right]+\left[\begin{array}{c}
\varepsilon_{3 x} \cos \psi_{3} \\
\varepsilon_{3 y} \sin \psi_{3} \\
\varepsilon_{3 z}
\end{array}\right]=\left[\begin{array}{c}
M_{x_{T}} \\
M_{y_{T}} \\
M_{z_{T}}
\end{array}\right]
$$

In order for a solution to exist to equation (6.64), one further condition must be imposed. If $\psi_{2}=0$, then equation (6.64) becomes:

$$
\left[\begin{array}{c}
\varepsilon_{1 x} \cos \psi_{1} \\
\varepsilon_{1 y} \sin \psi_{1} \\
\varepsilon_{1 z}
\end{array}\right]+\left[\begin{array}{c}
\varepsilon_{2 x} \\
0 \\
\varepsilon_{2 z}
\end{array}\right]+\left[\begin{array}{c}
\varepsilon_{3 x} \cos \psi_{3} \\
\varepsilon_{3 y} \sin \psi_{3} \\
\varepsilon_{3 z}
\end{array}\right]=\left[\begin{array}{c}
M_{x_{T}} \\
M_{y_{T}} \\
M_{z_{T}}
\end{array}\right]
$$

Equation (6.65) can be used to find $\psi_{1}$ and $\psi_{3}$ numerically.

If the $\psi$ components are held constant, equation (6.60) can be simplified by rewriting equations (6.57), (6.58), and (6.59) such that:

$$
\begin{gathered}
{\left[\begin{array}{ccc}
0 & -r_{1 z} & r_{1 y} \\
r_{1 z} & 0 & -r_{1 x} \\
-r_{1 y} & r_{1 x} & 0
\end{array}\right]\left[\begin{array}{c}
\cos \psi_{1} \\
\sin \psi_{1} \\
1
\end{array}\right] \Delta T_{1}=\left[\begin{array}{c}
-r_{1 z} \Delta T_{1}+r_{1 y} \Delta T_{1} \\
r_{1 z} \Delta T_{1}-r_{1 x} \Delta T_{1} \\
-r_{1 y} \Delta T_{1}+r_{1 x} \Delta T_{1}
\end{array}\right]\left[\begin{array}{c}
\cos \psi_{1} \\
\sin \psi_{1} \\
1
\end{array}\right]=\left[\begin{array}{c}
\varepsilon_{1 x} \\
\varepsilon_{1 y} \\
\varepsilon_{1 z}
\end{array}\right]} \\
{\left[\begin{array}{ccc}
0 & -r_{2 z} & 0 \\
r_{2 z} & 0 & -r_{2 x} \\
0 & r_{2 x} & 0
\end{array}\right]\left[\begin{array}{c}
\cos \psi_{2} \\
\sin \psi_{2} \\
1
\end{array}\right] \Delta T_{2}=\left[\begin{array}{c}
-r_{2 z} \Delta T_{2} \\
r_{2 z} \Delta T_{2}-r_{2 x} \Delta T_{2} \\
r_{2 x} \Delta T_{2}
\end{array}\right]\left[\begin{array}{c}
\cos \psi_{2} \\
\sin \psi_{2} \\
1
\end{array}\right]=\left[\begin{array}{c}
\varepsilon_{2 x} \\
\varepsilon_{2 y} \\
\varepsilon_{2 z}
\end{array}\right]} \\
{\left[\begin{array}{ccc}
0 & -r_{3 z} & r_{3 y} \\
r_{3 z} & 0 & -r_{3 x} \\
-r_{3 y} & r_{3 x} & 0
\end{array}\right]\left[\begin{array}{c}
\cos \psi_{3} \\
\sin \psi_{3} \\
1
\end{array}\right] \Delta T_{3}=\left[\begin{array}{c}
-r_{3 z} \Delta T_{3}+r_{3 y} \Delta T_{3} \\
r_{3 z} \Delta T_{3}-r_{3 x} \Delta T_{3} \\
-r_{3 y} \Delta T_{3}+r_{3 x} \Delta T_{3}
\end{array}\right]\left[\begin{array}{c}
\cos \psi_{3} \\
\sin \psi_{3} \\
1
\end{array}\right]=\left[\begin{array}{c}
\varepsilon_{3 x} \\
\varepsilon_{3 y} \\
\varepsilon_{3 z}
\end{array}\right]}
\end{gathered}
$$

yielding:

$$
\left[\begin{array}{l}
\varepsilon_{1 x} \\
\varepsilon_{1 y} \\
\varepsilon_{1 z}
\end{array}\right]\left[\begin{array}{l}
\cos \theta_{1} \\
\cos \theta_{1} \\
\sin \theta_{1}
\end{array}\right]+\left[\begin{array}{l}
\varepsilon_{2 x} \\
\varepsilon_{2 y} \\
\varepsilon_{2 z}
\end{array}\right]\left[\begin{array}{l}
\cos \theta_{2} \\
\cos \theta_{2} \\
\sin \theta_{2}
\end{array}\right]+\left[\begin{array}{l}
\varepsilon_{3 x} \\
\varepsilon_{3 y} \\
\varepsilon_{3 z}
\end{array}\right]\left[\begin{array}{c}
\cos \theta_{3} \\
\cos \theta_{3} \\
\sin \theta_{3}
\end{array}\right]=\left[\begin{array}{l}
M_{x_{T}} \\
M_{y_{T}} \\
M_{z_{T}}
\end{array}\right]
$$

Equation (6.69) can be used to determine $\theta_{1}, \theta_{2}$, and $\theta_{3}$ numerically. 


\section{Chapter 7: Analysis of Engine Compensation for Specific Actuator Failures}

The complete governing equation from equation (3.17) and (6.1) is

$$
\begin{aligned}
\sum_{i=1}^{n}\left\{\left[\begin{array}{ccc}
0 & -r_{i z} & r_{i y} \\
r_{i z} & 0 & -r_{i x} \\
-r_{i y} & r_{i x} & 0
\end{array}\right]_{B}\left[\begin{array}{c}
\cos \psi_{i} \cos \theta_{i} \\
\sin \psi_{i} \cos \theta_{i} \\
\sin \theta_{i}
\end{array}\right]_{B} \Delta T_{i}\right\} \\
=\sum_{j=1}^{m}\left\{\left[\begin{array}{l}
\left(M_{x \delta_{j}}\right)_{N} \\
\left(M_{y \delta_{j}}\right)_{N} \\
\left(M_{z \delta_{j}}\right)_{N}
\end{array}\right]_{B} \delta_{j C}-\left[\begin{array}{l}
\left(M_{x \delta_{j}}\right)_{F} \\
\left(M_{y \delta_{j}}\right)_{F} \\
\left(M_{z \delta_{j}}\right)_{F}
\end{array}\right]_{B} \delta_{j F}\right\}
\end{aligned}
$$

If the aerodynamic control surface $\mathrm{j}$ is stuck at a deflection $\delta_{j F}$. This means that the aerodynamic characteristics of the control surface are not altered and the following holds true:

$$
\frac{d \vec{M}_{N j}}{d \delta_{j}}=\frac{d \vec{M}_{F j}}{d \delta_{j}}
$$

and the moment due to the failure is:

$$
\vec{M}_{F j}=\frac{d \vec{M}_{N j}}{d \delta_{j}}\left(\delta_{j C}-\delta_{j F}\right)
$$

Equation (7.1) becomes

$$
\sum_{i=1}^{n}\left\{\left[\begin{array}{ccc}
0 & -r_{i z} & r_{i y} \\
r_{i z} & 0 & -r_{i x} \\
-r_{i y} & r_{i x} & 0
\end{array}\right]_{B}\left[\begin{array}{c}
\cos \psi_{i} \cos \theta_{i} \\
\sin \psi_{i} \cos \theta_{i} \\
\sin \theta_{i}
\end{array}\right]_{B} \Delta T_{i}\right\}=\sum_{j=1}^{m}\left\{\left(\delta_{j C}-\delta_{j F}\right)\left[\begin{array}{l}
\left(M_{x \delta_{j}}\right)_{N} \\
\left(M_{y \delta_{j}}\right)_{N} \\
\left(M_{z \delta_{j}}\right)_{N}
\end{array}\right]_{B}\right\}
$$

For the following discussion it is assumed that the aircraft is equipped with two identical engines $(n=2$, left $=L$, right $=R)$ located symmetrically such that 


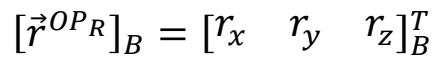

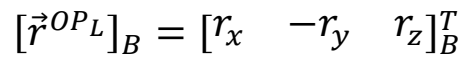

$$
\begin{aligned}
& {\left[\Delta \vec{T}_{R}\right]_{B}=\left[\begin{array}{c}
\Delta T_{x R} \\
\Delta T_{y R} \\
\Delta T_{z R}
\end{array}\right]_{B}=L_{B T_{R}}\left[\Delta \vec{T}_{R}\right]_{T_{R}}=\left[\begin{array}{c}
\cos \psi_{R} \cos \theta_{R} \\
\sin \psi_{R} \cos \theta_{R} \\
\sin \theta_{R}
\end{array}\right]_{B} \Delta T_{R}=\left[\begin{array}{c}
\varepsilon_{1 R} \\
\varepsilon_{2 R} \\
\varepsilon_{3 R}
\end{array}\right]_{B} \Delta T_{R}} \\
& {\left[\Delta \vec{T}_{L}\right]_{B}=\left[\begin{array}{c}
\Delta T_{x L} \\
\Delta T_{y L} \\
\Delta T_{z L}
\end{array}\right]_{B}=L_{B T_{L}}\left[\Delta \vec{T}_{L}\right]_{T_{L}}=\left[\begin{array}{c}
\cos \psi_{L} \cos \theta_{L} \\
\sin \psi_{L} \cos \theta_{L} \\
\sin \theta_{L}
\end{array}\right]_{B} \Delta T_{L}=\left[\begin{array}{c}
\varepsilon_{1 L} \\
\varepsilon_{2 L} \\
\varepsilon_{3 L}
\end{array}\right]_{B} \Delta T_{L}}
\end{aligned}
$$

An additional discussion is presented in Chapter 9:Appendix A: for an aircraft equipped with three identical engines $(n=3$, left $=L$, center $=C$, right $=R)$ located symmetrically.

\subsection{Elevator Failure}

If the assumption is made that the left elevator is locked at a deflection $\delta_{e L F}$, then the failure will affect the pitching moment and the rolling moment. It is assumed that the effects on the yawing moment are negligible. Under these conditions and omitting the subscript $\mathrm{N}$ for simplicity, equation (7.4) becomes

$$
\begin{gathered}
{\left[\begin{array}{ccc}
0 & -r_{z} & r_{y} \\
r_{z} & 0 & -r_{x} \\
-r_{y} & r_{x} & 0
\end{array}\right]_{B}\left[\begin{array}{c}
\Delta T_{x R} \\
\Delta T_{y R} \\
\Delta T_{z R}
\end{array}\right]_{B}+\left[\begin{array}{ccc}
0 & -r_{z} & -r_{y} \\
r_{z} & 0 & -r_{x} \\
r_{y} & r_{x} & 0
\end{array}\right]_{B}\left[\begin{array}{c}
\Delta T_{x L} \\
\Delta T_{y L} \\
\Delta T_{z L}
\end{array}\right]_{B}} \\
=\left(\delta_{e L C}-\delta_{e L F}\right)\left[\begin{array}{c}
M_{x \delta_{e L}} \\
M_{y \delta_{e L}} \\
0
\end{array}\right]_{B}
\end{gathered}
$$

Furthermore

$$
\left[\begin{array}{c}
-r_{z}\left(\Delta T_{y R}+\Delta T_{y L}\right)+r_{y}\left(\Delta T_{z R}-\Delta T_{z L}\right) \\
r_{z}\left(\Delta T_{x R}+\Delta T_{x L}\right)-r_{x}\left(\Delta T_{z R}+\Delta T_{z L}\right) \\
-r_{y}\left(\Delta T_{x R}-\Delta T_{x L}\right)+r_{x}\left(\Delta T_{y R}+\Delta T_{y L}\right)
\end{array}\right]_{B}=\left(\delta_{e L C}-\delta_{e L F}\right)\left[\begin{array}{c}
M_{x \delta_{e L}} \\
M_{y \delta_{e L}} \\
0
\end{array}\right]_{B}
$$


or using equations (7.7) and (7.8), equation (7.10) becomes

$$
\begin{gathered}
{\left[\begin{array}{c}
-r_{z}\left(\Delta T_{R} \varepsilon_{2 R}+\Delta T_{L} \varepsilon_{2 L}\right)+r_{y}\left(\Delta T_{R} \varepsilon_{3 R}-\Delta T_{L} \varepsilon_{3 L}\right) \\
r_{z}\left(\Delta T_{R} \varepsilon_{1 R}+\Delta T_{L} \varepsilon_{1 L}\right)-r_{x}\left(\Delta T_{R} \varepsilon_{3 R}+\Delta T_{L} \varepsilon_{3 L}\right) \\
-r_{y}\left(\Delta T_{R} \varepsilon_{1 R}-\Delta T_{L} \varepsilon_{1 L}\right)+r_{x}\left(\Delta T_{R} \varepsilon_{2 R}+\Delta T_{L} \varepsilon_{2 L}\right)
\end{array}\right]_{B}} \\
=\left(\delta_{e L C}-\delta_{e L F}\right)\left[\begin{array}{c}
M_{x \delta_{e L}} \\
M_{y \delta_{e L}} \\
0
\end{array}\right]_{B}
\end{gathered}
$$

This is a system of three equations and nine unknowns $\left(r_{x}, r_{y}, r_{z}, \Delta T_{R}, \Delta T_{L}, \theta_{R}, \psi_{R}, \theta_{L}\right.$, and $\left.\psi_{L}\right)$. If it is assumed that changing the direction of the thrust vector is not used for control, then the engine Euler angles are constant and respectively equal in magnitude and symmetric for the left and right engine. As a consequence:

$$
\varepsilon_{1 R}=\varepsilon_{1 L}=\varepsilon_{1}, \varepsilon_{2 R}=\varepsilon_{2 L}=\varepsilon_{2}, \text { and } \varepsilon_{3 R}=\varepsilon_{3 L}=\varepsilon_{3}
$$

and the number of unknowns - variables that can potentially be used for control - is reduced to five $\left(r_{x}, r_{y}, r_{z}, \Delta T_{R}\right.$, and $\left.\Delta T_{L}\right)$ :

$$
\left[\begin{array}{c}
-r_{z}\left(\Delta T_{R}+\Delta T_{L}\right) \varepsilon_{2}+r_{y}\left(\Delta T_{R}-\Delta T_{L}\right) \varepsilon_{3} \\
r_{z}\left(\Delta T_{R}+\Delta T_{L}\right) \varepsilon_{1}-r_{x}\left(\Delta T_{R}+\Delta T_{L}\right) \varepsilon_{3} \\
-r_{y}\left(\Delta T_{R}-\Delta T_{L}\right) \varepsilon_{1}+r_{x}\left(\Delta T_{R}-\Delta T_{L}\right) \varepsilon_{2}
\end{array}\right]_{B}=\left(\delta_{e L C}-\delta_{e L F}\right)\left[\begin{array}{c}
M_{x \delta_{e L}} \\
M_{y \delta_{e L}} \\
0
\end{array}\right]_{B}
$$

If the axes of the engines are parallel to the aircraft longitudinal axis, then $\varepsilon_{1}=1, \varepsilon_{2}=\varepsilon_{3}=0$, and the compensating engine moment is:

$$
\left[\sum_{i=1}^{2} \vec{M}_{e i}\right]_{B}=\left[\begin{array}{c}
0 \\
r_{z}\left(\Delta T_{R}+\Delta T_{L}\right) \\
-r_{y}\left(\Delta T_{R}-\Delta T_{L}\right)
\end{array}\right]_{B}
$$

In this case, the rolling effects of the failure cannot be compensated and an undesirable yawing moment is produced unless $\Delta T_{R}=\Delta T_{L}=\Delta T$. The pitching effects of the failure can be 
compensated if the engines are located off the body-axes horizontal plane $\left(r_{z} \neq 0\right)$. If this is true, then:

$$
2 r_{z} \Delta T=\left(\delta_{e L C}-\delta_{e L F}\right)
$$

To compensate for the rolling effects of the failure, $\varepsilon_{2} \neq 0$ and/or $\varepsilon_{3} \neq 0$. Collective thrust $\left(\Delta T_{R}=\Delta T_{L}=\Delta T\right)$ or differential thrust $\left(\Delta T_{R} \neq \Delta T_{L}\right)$ can be used. For the collective thrust case, equation (7.13) becomes:

$$
\left[\begin{array}{c}
-2 r_{z} \Delta T \varepsilon_{2} \\
r_{z} \Delta T \varepsilon_{1}-r_{x} \Delta T \varepsilon_{3} \\
0
\end{array}\right]_{B}=\left(\delta_{e L C}-\delta_{e L F}\right)\left[\begin{array}{c}
M_{x \delta_{e L}} \\
M_{y \delta_{e L}} \\
0
\end{array}\right]_{B}
$$

The following conditions result as necessary for rolling compensation:

$$
r_{z} \neq 0, \psi_{R}=\psi_{L} \neq 0, \text { and } \theta_{R}=\theta_{L} \neq \frac{\pi}{2}
$$

Assuming that the devices considered are the actual aircraft main propulsion system, then $\left|\psi_{R}\right|=\left|\psi_{L}\right| \neq \frac{\pi}{2}$ and $\varepsilon_{1} \neq 0$. Pitching compensation capabilities are maintained if:

$$
\theta_{R}=\theta_{L}=0 \text { or } r_{x}=0 \quad \Rightarrow \quad r_{x} \Delta T \varepsilon_{1}=\left(\delta_{e L C}-\delta_{e L F}\right) M_{y \delta_{e L}}
$$

or:

$$
r_{z} \varepsilon_{1} \neq r_{x} \varepsilon_{3} \quad \Rightarrow \quad\left(r_{z} \varepsilon_{1}-r_{x} \varepsilon_{3}\right) \Delta T=\left(\delta_{e L C}-\delta_{e L F}\right) M_{y \delta_{e L}}
$$

For the differential thrust case, considering that $\varepsilon_{1} \neq 0$ for the actual main aircraft propulsion system, the yawing effects are avoided if:

$$
r_{y} \varepsilon_{1}=r_{x} \varepsilon_{2}
$$


Assuming that the differential thrust control is symmetric, that is $\Delta T_{R}=-\Delta T_{L}=\Delta T$, then engine compensating moment becomes:

$$
\left[\sum_{i=1}^{2} \vec{M}_{e i}\right]_{B}=\left[\begin{array}{c}
2 r_{y} \Delta T \varepsilon_{3} \\
0 \\
0
\end{array}\right]_{B}
$$

The following conditions result as necessary for rolling compensation:

$$
r_{y} \neq 0 \text { and } \theta_{R}=\theta_{L} \neq 0
$$

In order to maintain pitching moment compensation capabilities, $\Delta T_{R} \neq \Delta T_{L}$ and equation (7.13) becomes:

$$
\left[\begin{array}{c}
-r_{z}\left(\Delta T_{R}+\Delta T_{L}\right) \varepsilon_{2}+r_{y}\left(\Delta T_{R}-\Delta T_{L}\right) \varepsilon_{3} \\
r_{z}\left(\Delta T_{R}+\Delta T_{L}\right) \varepsilon_{1}-r_{x}\left(\Delta T_{R}+\Delta T_{L}\right) \varepsilon_{3} \\
0
\end{array}\right]_{B}=\left(\delta_{e L C}-\delta_{e L F}\right)\left[\begin{array}{c}
M_{x \delta_{e L}} \\
M_{y \delta_{e L}} \\
0
\end{array}\right]_{B}
$$

The additional conditions for both rolling and pitching compensation result in:

$$
\begin{gathered}
r_{z}\left(\Delta T_{R}+\Delta T_{L}\right) \varepsilon_{2} \neq r_{y}\left(\Delta T_{R}-\Delta T_{L}\right) \varepsilon_{3} \\
r_{z} \varepsilon_{1} \neq r_{x} \varepsilon_{3}
\end{gathered}
$$

If the location and orientation of the two thrust vectors are fixed, then equation (7.23) can be used to solve for $\Delta T_{R}$ and $\Delta T_{L}$. Assuming that equation (7.24) and equation (7.25) are valid, this yields:

$$
\begin{aligned}
& \Delta T_{R}=\frac{1}{2}\left(\frac{\left(\delta_{e L C}-\delta_{e L F}\right) M_{y \delta_{e L}}}{r_{z} \varepsilon_{1}-r_{x} \varepsilon_{3}}-\frac{\left(\delta_{e L C}-\delta_{e L F}\right) M_{x \delta_{e L}}}{r_{z} \varepsilon_{2}-r_{y} \varepsilon_{3}}\right) \\
& \Delta T_{L}=\frac{1}{2}\left(\frac{\left(\delta_{e L C}-\delta_{e L F}\right) M_{y \delta_{e L}}}{r_{z} \varepsilon_{1}-r_{x} \varepsilon_{3}}+\frac{\left(\delta_{e L C}-\delta_{e L F}\right) M_{x \delta_{e L}}}{r_{z} \varepsilon_{2}-r_{y} \varepsilon_{3}}\right)
\end{aligned}
$$


If $\Delta T_{R}$ and $\Delta T_{L}$ are known as well as the moments produced by the failed elevator and assuming that there is no change in the thrust vector, then equation (7.13) can be utilized in order to find the engine positions. Solving equation (7.13) for $r_{x}, r_{y}$, and $r_{z}$ yields:

$$
\begin{aligned}
& r_{x}=\frac{\left(\delta_{e L C}-\delta_{e L F}\right) M_{x \delta_{e L}} \varepsilon_{1}\left(\Delta T_{R}+\Delta T_{L}\right)+\left(\delta_{e L C}-\delta_{e L F}\right) M_{y \delta_{e L}} \varepsilon_{2}\left(\Delta T_{R}-\Delta T_{L}\right)}{2 \varepsilon_{2} \varepsilon_{3} \Delta T_{L}\left(\Delta T_{R}+\Delta T_{L}\right)} \\
& r_{y}=\frac{\left(\delta_{e L C}-\delta_{e L F}\right) M_{x \delta_{e L}} \varepsilon_{1}\left(\Delta T_{R}+\Delta T_{L}\right)+\left(\delta_{e L C}-\delta_{e L F}\right) M_{y \delta_{e L}} \varepsilon_{2}\left(\Delta T_{R}-\Delta T_{L}\right)}{2 \varepsilon_{1} \varepsilon_{3} \Delta T_{L}\left(-\Delta T_{R}+\Delta T_{L}\right)} \\
& r_{z}=\frac{\left(\delta_{e L C}-\delta_{e L F}\right) M_{x \delta_{e L} \varepsilon_{1}}+\left(\delta_{e L C}-\delta_{e L F}\right) M_{y \delta_{e L}} \varepsilon_{2}}{2 \varepsilon_{1} \varepsilon_{2} \Delta T_{L}}
\end{aligned}
$$

\subsection{Aileron Failure}

If it is assumed that the left aileron is locked at a deflection $\delta_{a L F}$. The failure will affect primarily the rolling and to a lesser extent the yawing moment, while it is assumed that the effects on the pitching moment are negligible. Following the same procedure as for the elevator, an equation similar to equation (7.11) is obtained:

$$
\begin{gathered}
{\left[\begin{array}{c}
-r_{z}\left(\Delta T_{R} \varepsilon_{2 R}+\Delta T_{L} \varepsilon_{2 L}\right)+r_{y}\left(\Delta T_{R} \varepsilon_{3 R}-\Delta T_{L} \varepsilon_{3 L}\right) \\
r_{z}\left(\Delta T_{R} \varepsilon_{1 R}+\Delta T_{L} \varepsilon_{1 L}\right)-r_{x}\left(\Delta T_{R} \varepsilon_{3 R}+\Delta T_{L} \varepsilon_{3 L}\right) \\
-r_{y}\left(\Delta T_{R} \varepsilon_{1 R}-\Delta T_{L} \varepsilon_{1 L}\right)+r_{x}\left(\Delta T_{R} \varepsilon_{2 R}+\Delta T_{L} \varepsilon_{2 L}\right)
\end{array}\right]_{B}} \\
=\left(\delta_{a L C}-\delta_{a L F}\right)\left[\begin{array}{c}
M_{x \delta_{a L}} \\
0 \\
M_{z \delta_{a L}}
\end{array}\right]_{B}
\end{gathered}
$$

Assuming again that changing the direction of the thrust vector is not used for control and considering equation (7.12): 


$$
\left[\begin{array}{c}
-r_{z}\left(\Delta T_{R}+\Delta T_{L}\right) \varepsilon_{2}+r_{y}\left(\Delta T_{R}-\Delta T_{L}\right) \varepsilon_{3} \\
r_{z}\left(\Delta T_{R}+\Delta T_{L}\right) \varepsilon_{1}-r_{x}\left(\Delta T_{R}+\Delta T_{L}\right) \varepsilon_{3} \\
-r_{y}\left(\Delta T_{R}-\Delta T_{L}\right) \varepsilon_{1}+r_{x}\left(\Delta T_{R}-\Delta T_{L}\right) \varepsilon_{2}
\end{array}\right]_{B}=\left(\delta_{a L C}-\delta_{a L F}\right)\left[\begin{array}{c}
M_{x \delta_{a L}} \\
0 \\
M_{z \delta_{a L}}
\end{array}\right]_{B}
$$

Since a compensatory rolling moment must be produced, differential vertical and/or lateral thrust components are necessary. If it is assumed that differential symmetric thrust is used $\left(\Delta T_{R}=\right.$ $\left.-\Delta T_{L}=\Delta T\right)$, then:

$$
\left[\begin{array}{c}
2 r_{y} \Delta T \varepsilon_{3} \\
0 \\
-2 r_{y} \Delta T \varepsilon_{1}+2 r_{x} \Delta T \varepsilon_{2}
\end{array}\right]_{B}=\left(\delta_{a L C}-\delta_{a L F}\right)\left[\begin{array}{c}
M_{x \delta_{a L}} \\
0 \\
M_{z \delta_{a L}}
\end{array}\right]_{B}
$$

The additional conditions for rolling moment compensation are:

$$
\theta_{R}=\theta_{L} \neq 0 \text { and } r_{y} \neq 0
$$

Furthermore, yawing compensation is provided if:

$$
r_{y} \varepsilon_{1} \neq r_{x} \varepsilon_{2}
$$

If the location and orientation of the engine thrust vectors are fixed, then equation (7.33) can be used to solve for $\Delta T$. Assuming that equation (7.34) and (7.35) are valid, this yields:

$$
\Delta T=\frac{\left(\delta_{a L C}-\delta_{a L F}\right) M_{x \delta_{a L}}}{2 r_{y} \varepsilon_{3}}
$$

or:

$$
\Delta T=\frac{\left(\delta_{a L C}-\delta_{a L F}\right) M_{z \delta_{a L}}}{2 r_{x} \varepsilon_{2}-2 r_{y} \varepsilon_{1}}
$$




\subsection{Rudder Failure}

Assume that the rudder is locked at a deflection $\delta_{r F}$. The failure will affect primarily the yawing and to some extent the rolling moment, while it is assumed that the effects on the pitching moment are negligible. Following the same procedure as for the elevator failure, an equation similar to equation (7.11) is obtained as:

$$
\left[\begin{array}{c}
-r_{z}\left(\Delta T_{R} \varepsilon_{2 R}+\Delta T_{L} \varepsilon_{2 L}\right)+r_{y}\left(\Delta T_{R} \varepsilon_{3 R}-\Delta T_{L} \varepsilon_{3 L}\right) \\
r_{z}\left(\Delta T_{R} \varepsilon_{1 R}+\Delta T_{L} \varepsilon_{1 L}\right)-r_{x}\left(\Delta T_{R} \varepsilon_{3 R}+\Delta T_{L} \varepsilon_{3 L}\right) \\
-r_{y}\left(\Delta T_{R} \varepsilon_{1 R}-\Delta T_{L} \varepsilon_{1 L}\right)+r_{x}\left(\Delta T_{R} \varepsilon_{2 R}+\Delta T_{L} \varepsilon_{2 L}\right)
\end{array}\right]_{B}=\left(\delta_{r C}-\delta_{r F}\right)\left[\begin{array}{c}
M_{x \delta_{r}} \\
0 \\
M_{z \delta_{r}}
\end{array}\right]_{B}
$$

Assuming again that changing the direction of the thrust vector is not used for control and considering equation (7.12):

$$
\left[\begin{array}{c}
-r_{z}\left(\Delta T_{R}+\Delta T_{L}\right) \varepsilon_{2}+r_{y}\left(\Delta T_{R}-\Delta T_{L}\right) \varepsilon_{3} \\
r_{z}\left(\Delta T_{R}+\Delta T_{L}\right) \varepsilon_{1}-r_{x}\left(\Delta T_{R}+\Delta T_{L}\right) \varepsilon_{3} \\
-r_{y}\left(\Delta T_{R}-\Delta T_{L}\right) \varepsilon_{1}+r_{x}\left(\Delta T_{R}-\Delta T_{L}\right) \varepsilon_{2}
\end{array}\right]_{B}=\left(\delta_{r C}-\delta_{r F}\right)\left[\begin{array}{c}
M_{x \delta_{r}} \\
0 \\
M_{z \delta_{r}}
\end{array}\right]_{B}
$$

Since a compensatory yawing moment must be produced primarily, differential longitudinal thrust components are sufficient provided the distance from the engine to the vertical plane of symmetry is large enough. If differential symmetric thrust $\left(\Delta T_{R}=-\Delta T_{L}=\Delta T\right)$ is used, then:

$$
\left[\begin{array}{c}
2 r_{y} \Delta T \varepsilon_{3} \\
0 \\
-2\left(r_{y} \varepsilon_{1}-r_{x} \varepsilon_{2}\right) \Delta T
\end{array}\right]_{B}=\left(\delta_{r C}-\delta_{r F}\right)\left[\begin{array}{c}
M_{x \delta_{r}} \\
0 \\
M_{z \delta_{r}}
\end{array}\right]_{B}
$$

Equation (7.40) shows that if the location and the orientation of the two thrust vectors are fixed then differential thrust commands can be determined in order to produce compensation of the yawing moment. The necessary thrust is: 


$$
\Delta T=\frac{\left(\delta_{r C}-\delta_{r F}\right) M_{z \delta_{r}}}{-2\left(r_{y} \varepsilon_{1}-r_{x} \varepsilon_{2}\right)}
$$

However, it might be necessary to compensate for the residual moment on the lateral channel by using the aileron.

If the engine thrust differential is known as well as the moments produced by the failed rudder and assuming that there is no change in the thrust vector, then equation (7.39) can be used to determine the engine positions. Solving for $r_{x}, r_{y}$, and $r_{z}$ yields:

$$
\begin{aligned}
& r_{x}=\frac{\delta_{r C}-\delta_{r F}}{\left(\Delta T_{R}-\Delta T_{L}\right) \varepsilon_{2}}\left[2 M_{z \delta_{r}}+\frac{M_{x \delta_{r}} \varepsilon_{1}}{\varepsilon_{3}}\right]-1 \\
& r_{y}=\frac{\left(\delta_{r C}-\delta_{r F}\right)\left(M_{x \delta_{r}} \varepsilon_{1}+M_{z \delta_{r}} \varepsilon_{3}\right)}{\left(\Delta T_{R}-\Delta T_{L}\right) \varepsilon_{1} \varepsilon_{3}}-\frac{\varepsilon_{2}}{\varepsilon_{1}} \\
& r_{z}=\frac{\delta_{r C}-\delta_{r F}}{\left(\Delta T_{R}-\Delta T_{L}\right) \varepsilon_{2}}\left[\frac{2 M_{z \delta_{r}} \varepsilon_{3}}{\varepsilon_{1}}+M_{x \delta_{r}}\right]-\frac{\varepsilon_{3}}{\varepsilon_{1}}
\end{aligned}
$$




\section{Chapter 8: Simulation Results and Discussion}

\subsection{Nominal Flight Condition}

The nominal conditions flight test is based on the dynamics of a Boeing 747 model flying at an altitude of 10,668 meters (36,000 feet) above sea level with negligible wind and turbulence effects. The aircraft is flying at an average speed of 250 meters per second (559 miles per hour). The engines are numbered from farthest location on the pilot's left to the farthest location on the pilot's right. Therefore, for this aircraft there are two engines on the pilot's left with Engine \#1 being closer to the wingtip and Engine \#2 being closer to the aircraft fuselage. Engine \#3 is closer to the fuselage on the pilot's right and Engine \#4 is closer to the wingtip on the pilot's right. The engine locations are assumed to be symmetrical about the longitudinal plane such that $\left[\begin{array}{lll}r_{1 x} & r_{1 y} & r_{1 z}\end{array}\right]=\left[\begin{array}{lll}r_{4 x} & -r_{4 y} & r_{4 z}\end{array}\right]$ and $\left[\begin{array}{lll}r_{2 x} & r_{2 y} & r_{2 z}\end{array}\right]=\left[\begin{array}{lll}r_{3 x} & -r_{3 y} & r_{3 z}\end{array}\right]$. This assumption is held throughout all of the experiments.

The nominal flight consists of five main sections. For the first 60 seconds of flight no pilot input is given to the aircraft. Between the 61 second mark and the 120 second mark a roll doublet is executed. During the course of the next 60 seconds a pitch doublet is performed. A yaw doublet is then carried out between the 181 second mark and 240 second mark of the experiment. Finally, a throttle doublet completes the set of nominal conditions tests.

For the purposes of the nominal test flight and all other test flights within this thesis, two factors must be defined. A "controllable" flight is one where the aircraft is capable of maintaining relatively level flight through either the FLC, pilot commands through the joystick, or a combination of both. An "uncontrollable" flight is one where the aircraft's roll, pitch, 
and/or yaw moments generated by the aircraft are greater than what the operating control surfaces and FLC can compensate.

The control surface deflections for the nominal flight test can be seen in Figure 8-1. The roll, pitch, and yaw doublets described above are evident. These deflections are supported by their corresponding rates viewed in Figure 8-2. The pilot commanded throttle input to each engine and the resulting throttle response is in Figure 8-3. Here, the final set of nominal commands for the throttle as described above is evident.

Overall, the aircraft is responsive towards the pilot's commanded control surface deflections and throttle input. In Figure 8-1 the left and right elevator deflections are uniform which is why only the right elevator deflections are apparent on the plot. Likewise, the pilot throttle command is equivalent for engine and the FLC throttle command is equivalent for each engine and thus only Engine \#4's values are visible in Figure 8-3.
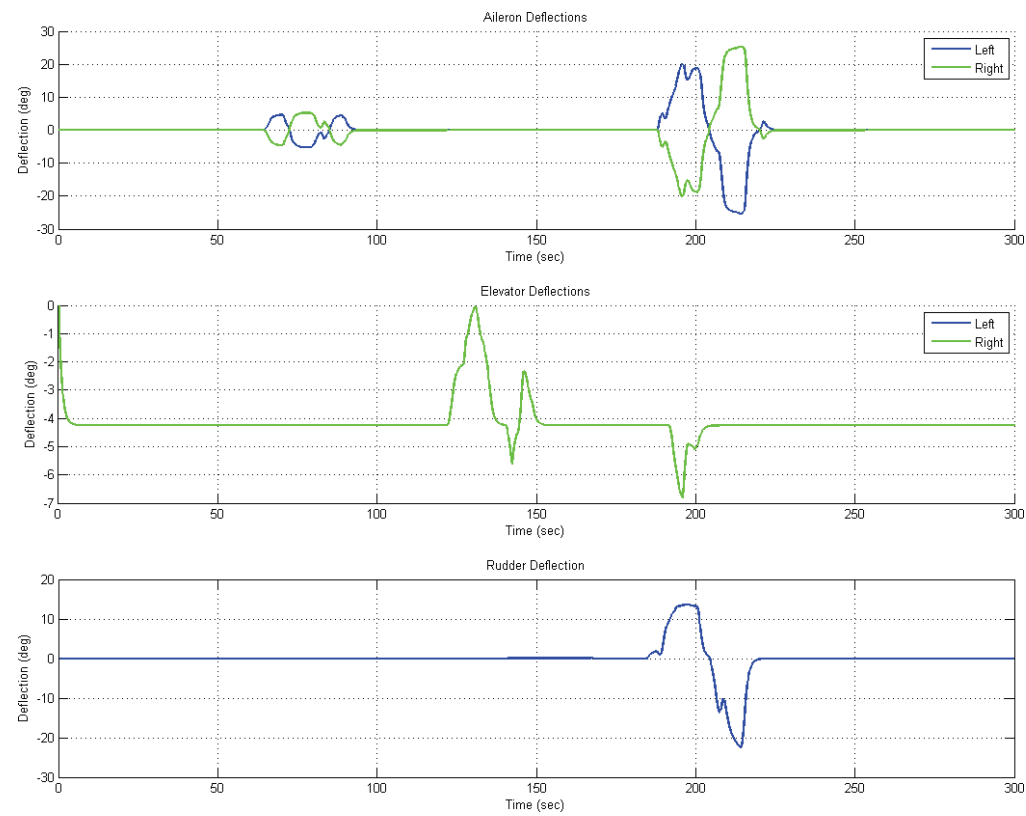

Figure 8-1: Control Surface Deflections for the Elevators, Ailerons, and Rudder for the Nominal Conditions Test 

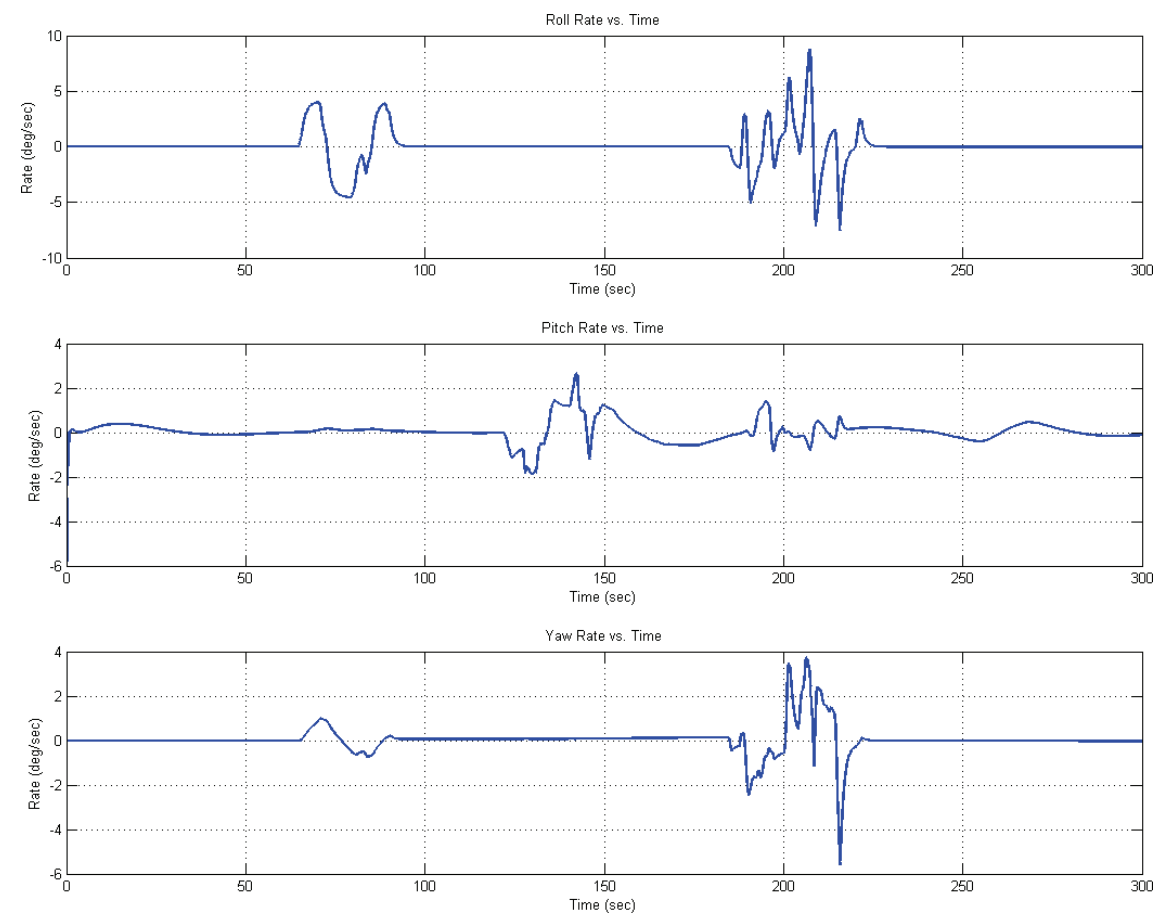

Figure 8-2: Roll, Pitch, and Yaw Rates for the Nominal Conditions Test
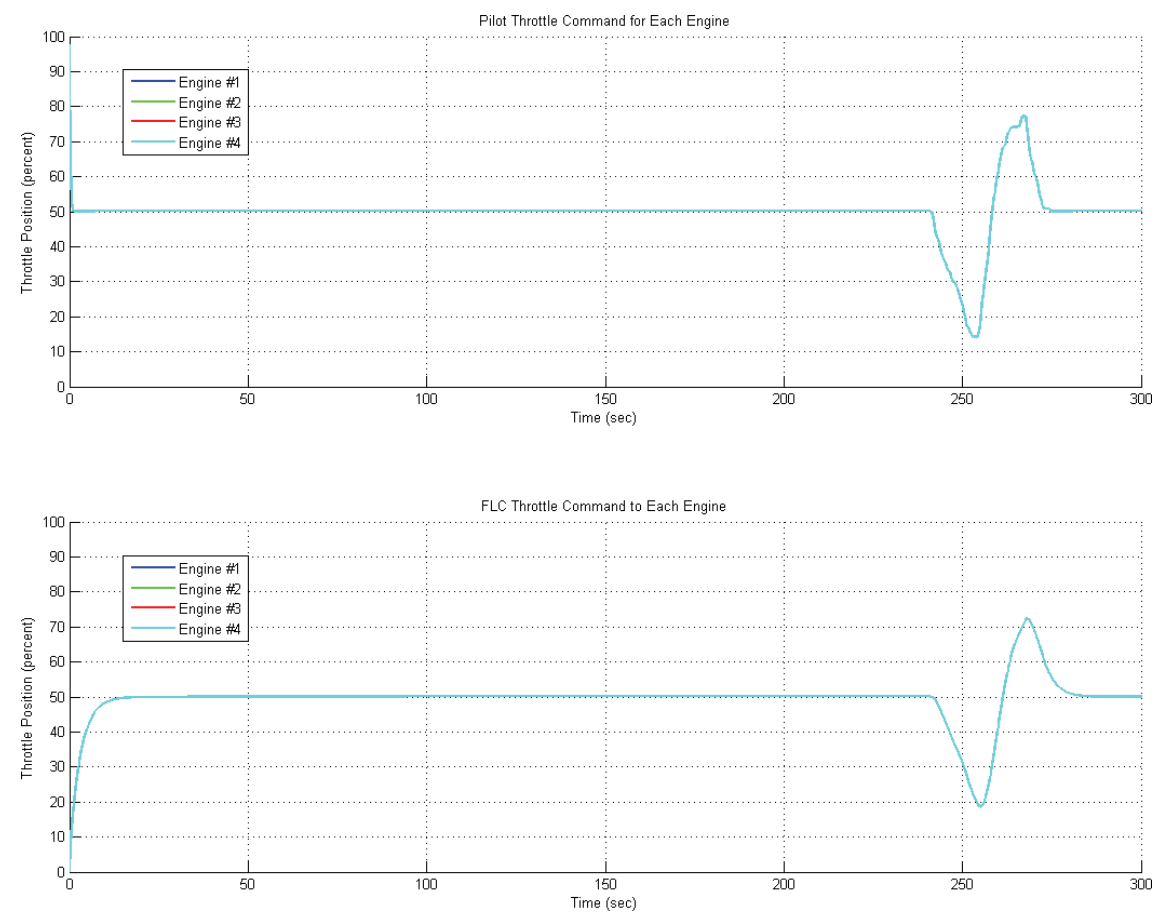

Figure 8-3: Pilot Throttle Command and the FLC Throttle Command to Each Engine for the Nominal Conditions Test 


\subsection{Elevator Failure}

The model was exposed to two levels of elevator failure. Additionally, it was tested at each level of failure with the FLC engaged and disengaged. The magnitudes of failure considered were a left stuck elevator actuator at -10 degrees and +10 degrees. The elevator trim position is -4.25 degrees indicating that the -10 degree test is actually an elevator actuator stuck at -5.75 degrees from the trim position. Likewise, the +10 degree test has the elevator actuator stuck +14.25 degrees from the trim position. During each test, the aircraft was subjected to the indicated failure at the 60 second mark allowing for a small period of nominal flight data to be gathered as well as the abnormal condition data. The engine locations were assumed symmetrical as in the nominal flight test. Furthermore, symmetric thrust about the longitudinal plane was utilized as was differential thrust about the lateral plane.

When the aircraft was exposed to the -10 degree stuck left elevator actuator it was controllable while the FLC was engaged and disengaged. While the FLC was engaged, pilot input was not required in order to maintain altitude by canceling the moment induced by the failed actuator as perceived Figure 8-4. This is equivalent to all the control surfaces being locked at the trim positions with the left elevator being locked at a non-trim position. Conversely, when the controller was disengaged the pilot needed to maintain a new trim position in order to achieve level flight. Therefore, in an emergency situation where the hydraulic pumps controlling the control surface actuators fail, the FLC would be capable of assisting the pilot in maintaining a controllable flight envelope. The FLC throttle commands sent to the engine can be seen in Figure 8-5. The throttle commands for Engine \#1 and Engine \#4 are equal as are the throttle commands for Engine \#2 and Engine \#3. This is why only two throttle commands are visible on the graph. The roll rate, pitch rate, and yaw rate while the FLC was engaged and 
disengaged are displayed in Figure 8-6. The pitch rate while the FLC was engaged has fewer peaks indicating a smoother flight than when the controller was disengaged.

When the aircraft was subjected to the +10 degree stuck left elevator actuator, it was controllable with and without the FLC engaged. The difference in the amount of pilot input required as seen through the elevator control surface deflection differences in Figure 8-7. The average amount of right elevator deflection while the FLC was engaged is -12.5 degrees and while the controller was disengaged the average is -14.2 degrees. The throttle commands to the engines were near $100 \%$ as seen in Figure 8-8. As with the -10 degree failure case above, while the FLC was engaged the pitch rate has a smoother profile than when the controller was disengaged as observed in Figure 8-9.
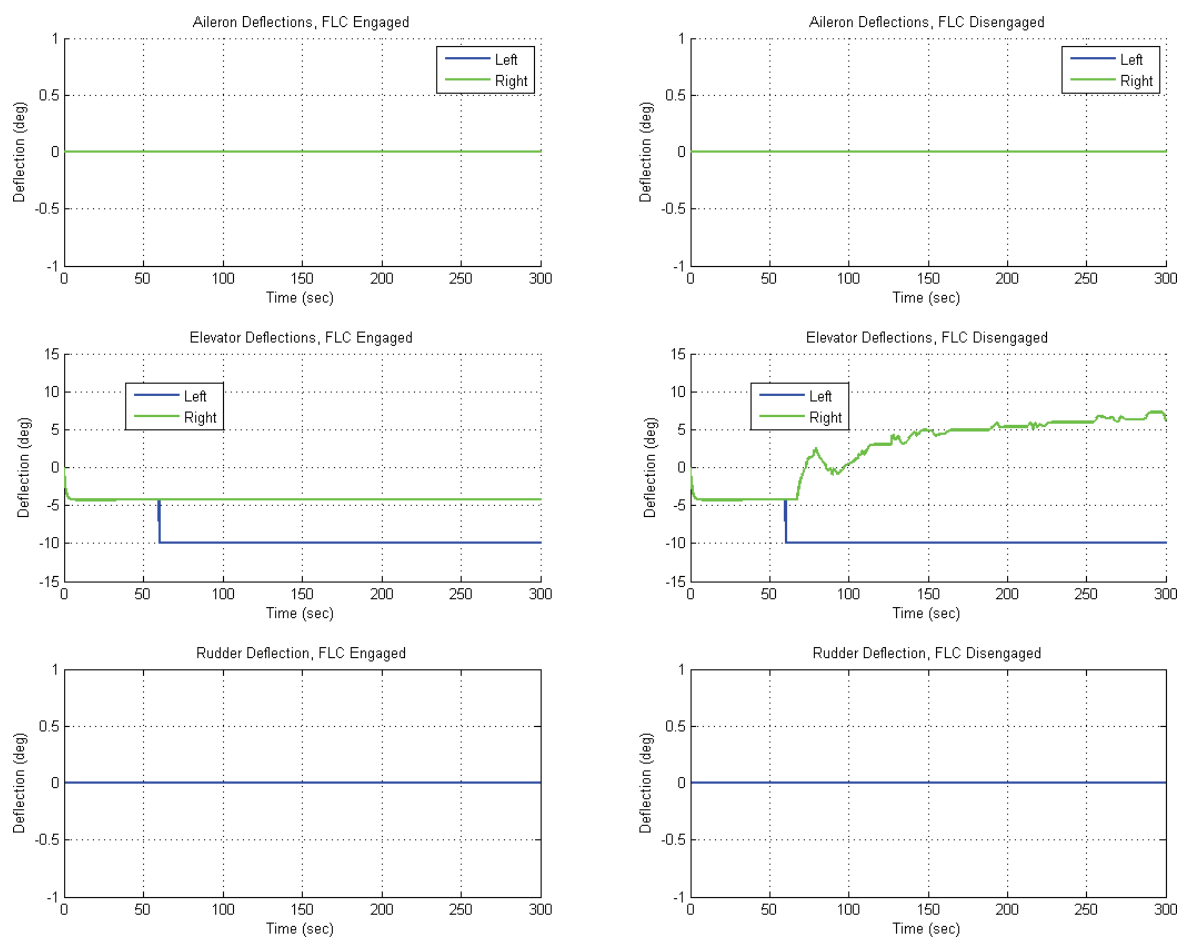

Figure 8-4: Control Surface Deflections for the -10 Degree Left Elevator Failure Case with the FLC Engaged and Disengaged 

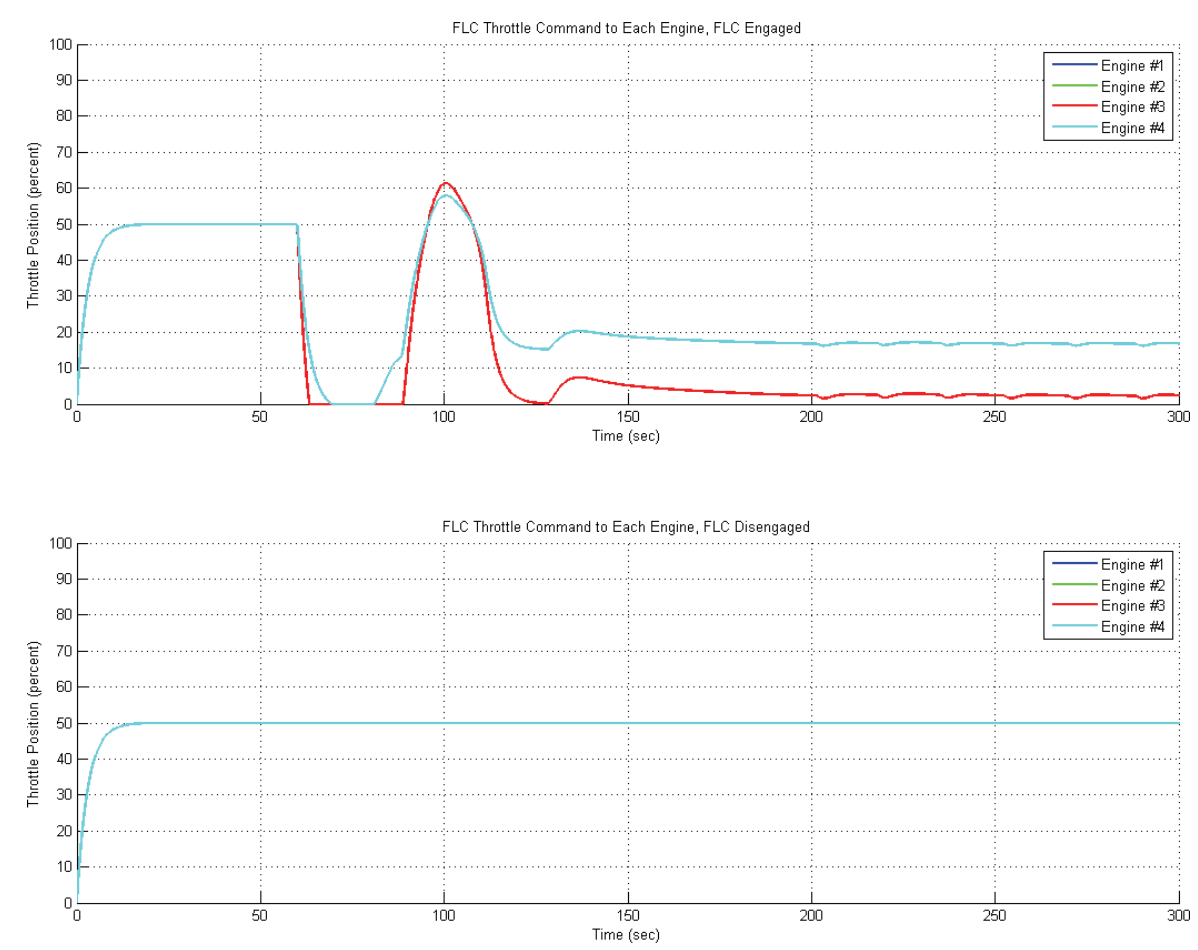

Figure 8-5: Throttle Commands for the -10 Degree Left Elevator Failure Case with the FLC Engaged and Disengaged
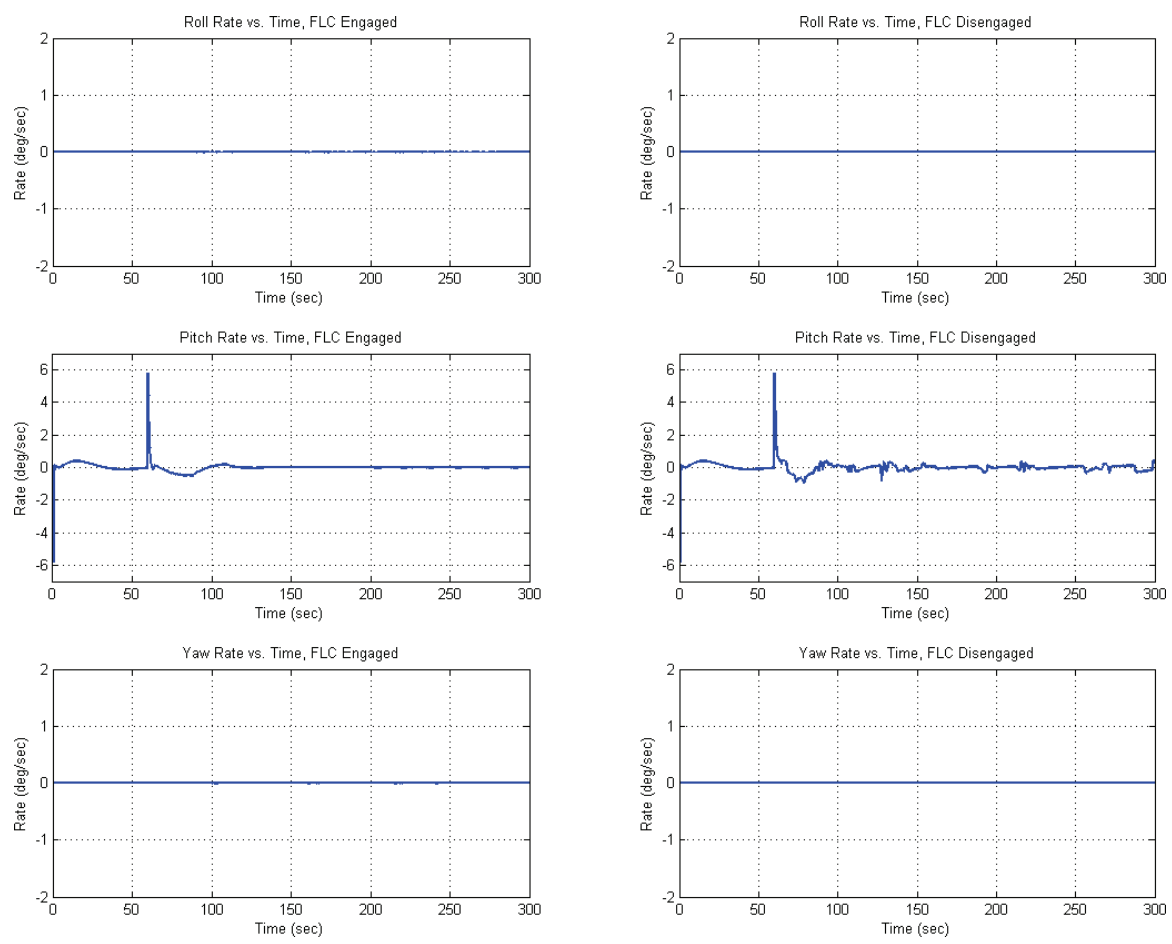

Figure 8-6: Roll, Pitch, and Yaw Rates for the -10 Degree Left Elevator Failure Case with the FLC Engaged and Disengaged 

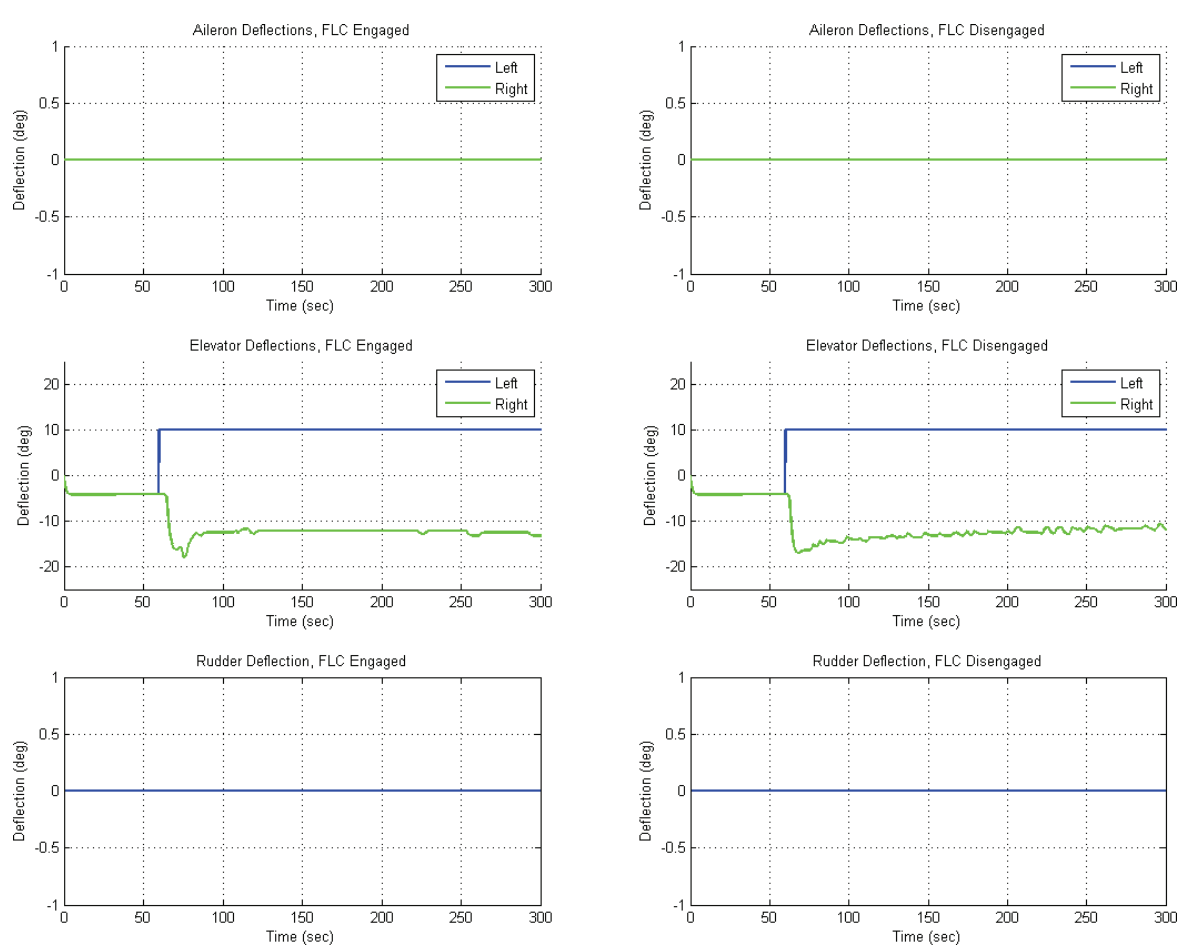

Figure 8-7: Control Surface Deflections for the +10 Degree Left Elevator Failure Case with the FLC Engaged and Disengaged
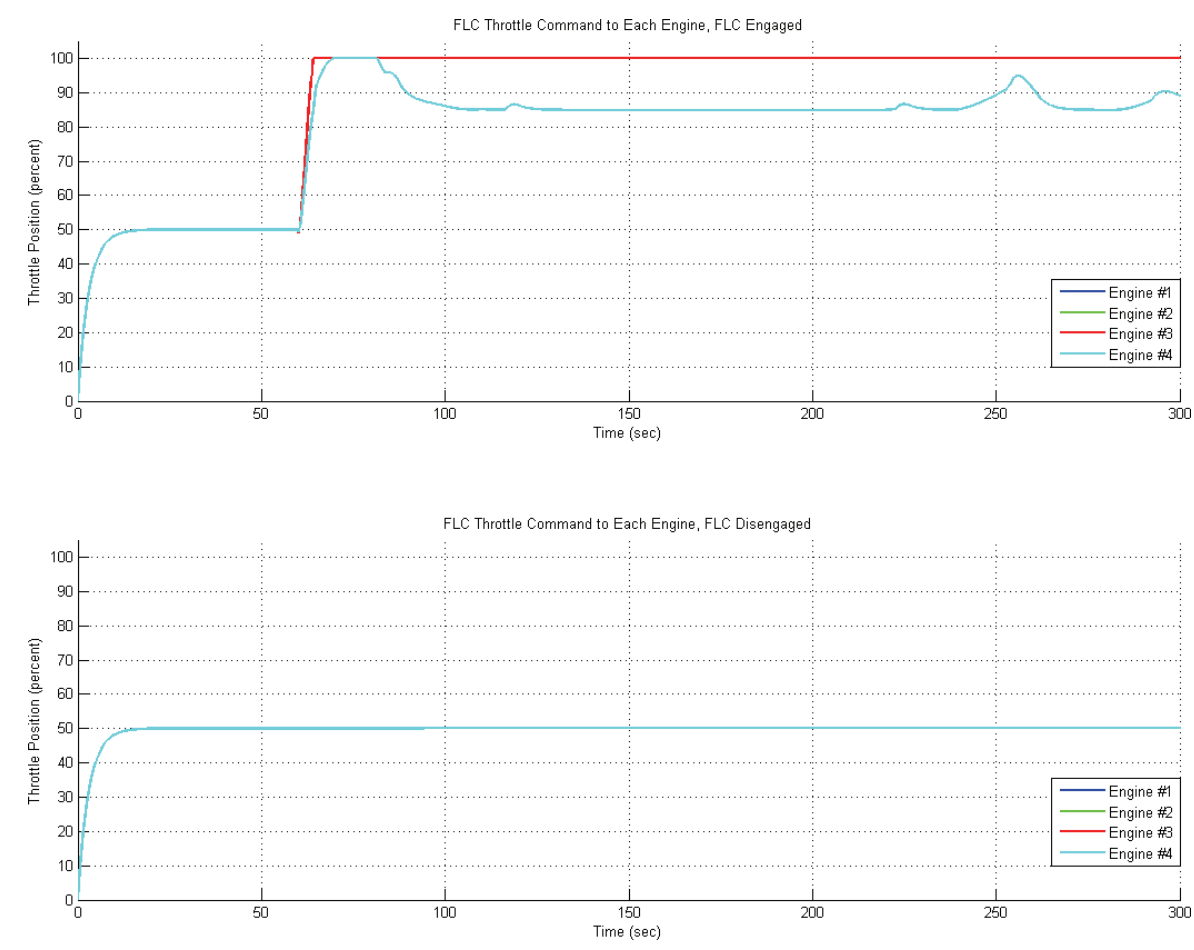

Figure 8-8: Throttle Commands for the +10 Degree Left Elevator Failure Case with the FLC Engaged and Disengaged 

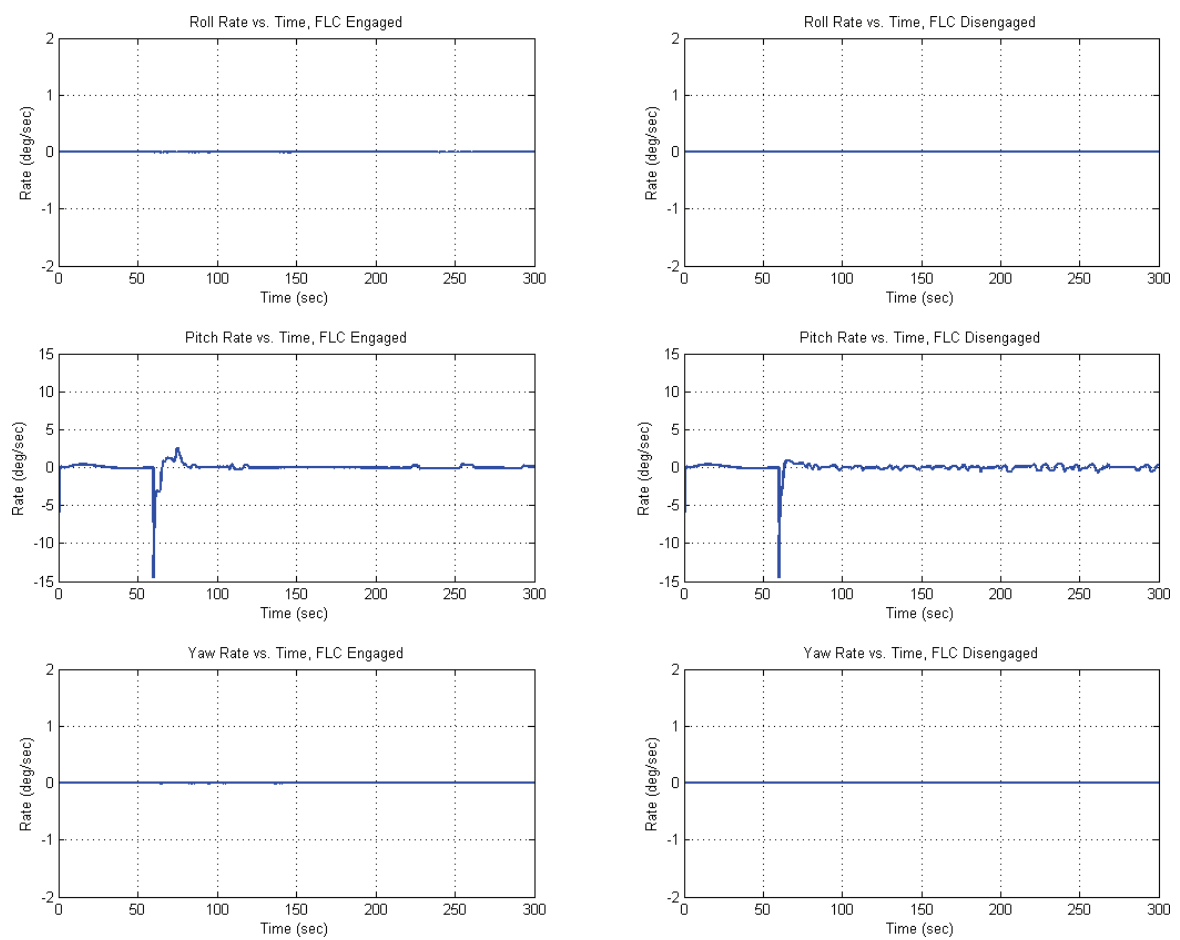

Figure 8-9: Roll, Pitch, and Yaw Rates for the +10 Degree Left Elevator Failure Case with the FLC Engaged and Disengaged

\subsection{Aileron Failure}

The model was exposed to two levels of aileron failure. Additionally, it was tested at each level of failure with the FLC engaged and disengaged. The magnitudes of failure considered were a left stuck aileron actuator at +10 degrees and +20 degrees. During each test, the aircraft was subjected to the indicated failure at the 60 second mark such that there is some nominal flight data before the abnormal condition. The engine locations were assumed symmetrical as in the nominal flight test. Furthermore, differential thrust about the longitudinal plane of symmetry was utilized.

When the aircraft was exposed to the +10 degree stuck left aileron actuator it was controllable while the FLC was engaged and disengaged. Of particular note is that the amount of pilot input, and thus aileron control surface deflection, required for level flight varied greatly as 
seen in Figure 8-10. In fact, while the FLC was engaged the aircraft did not require any pilot input to maintain level flight. This allows the pilot to maintain a greater flight envelope under the failure scenario. As viewed in Figure 8-11, the controller sent varied throttle commands to the engines in order to counter the moment induced by the stuck aileron. The FLC is capable of roll control because equation (7.34) holds true since $\theta_{R}=\theta_{L}=2.5^{\circ}$. Furthermore, the FLC was capable of dampening the roll rate observed in Figure 8-12.

When the aircraft was exposed to the +20 degree stuck left aileron actuator, it was still controllable while the FLC was engaged and disengaged. As with the +10 degree test, the pilot was not required to give any input into the system while the FLC was engaged whereas the pilot needed to maintain a constant aileron command to maintain level flight as viewed in Figure 8-13. The throttle command sent to each engine by the FLC can be seen in Figure 8-14. The magnitude of the commands was greater than for the +10 degree test but throttle command still has not saturated the system. This means that the controller can handle a larger failure if needed and the pilot still has some control authority over the throttle level. Furthermore, the FLC was capable of dampening the roll rate as observed in Figure 8-15. 

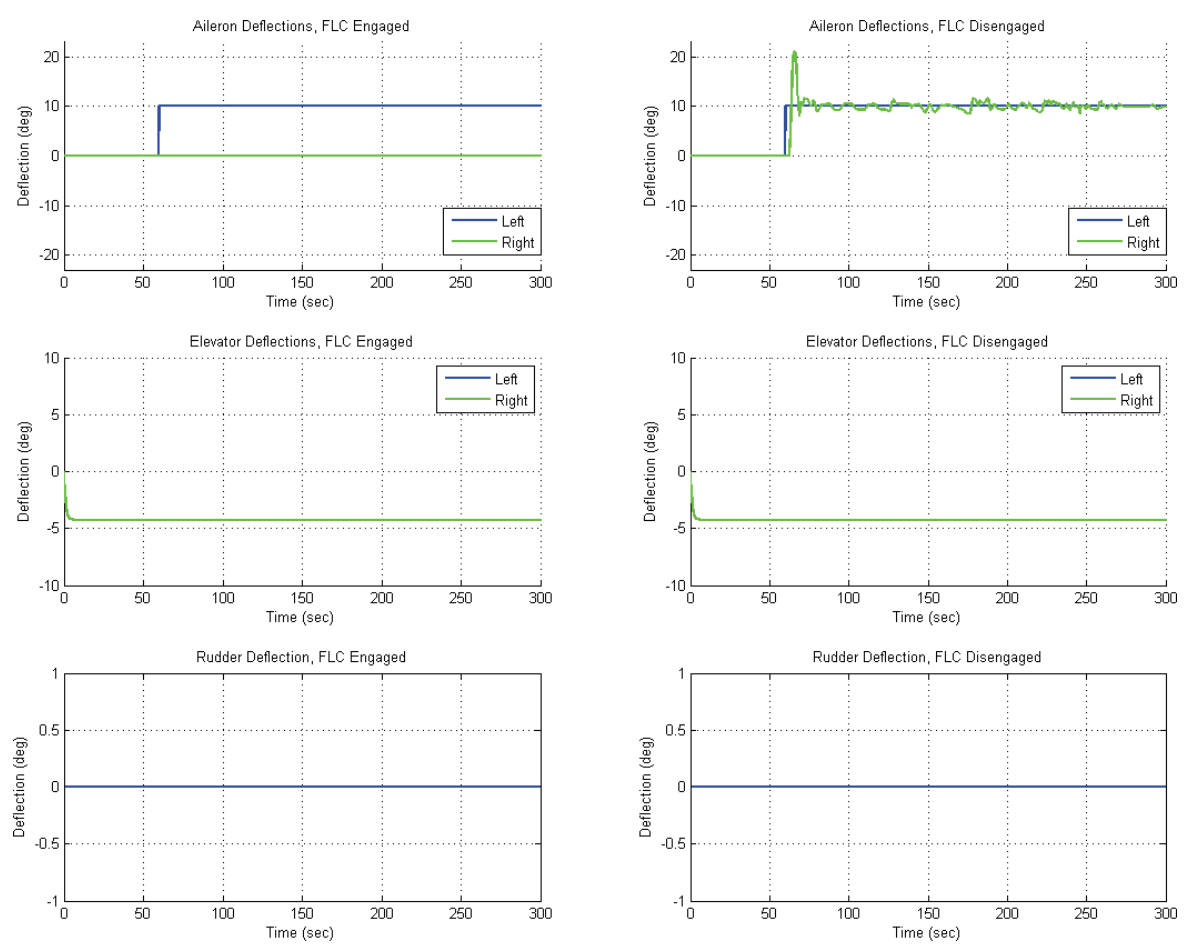

Figure 8-10: Control Surface Deflections for the +10 Degree Left Aileron Failure Case with the FLC Engaged and Disengaged
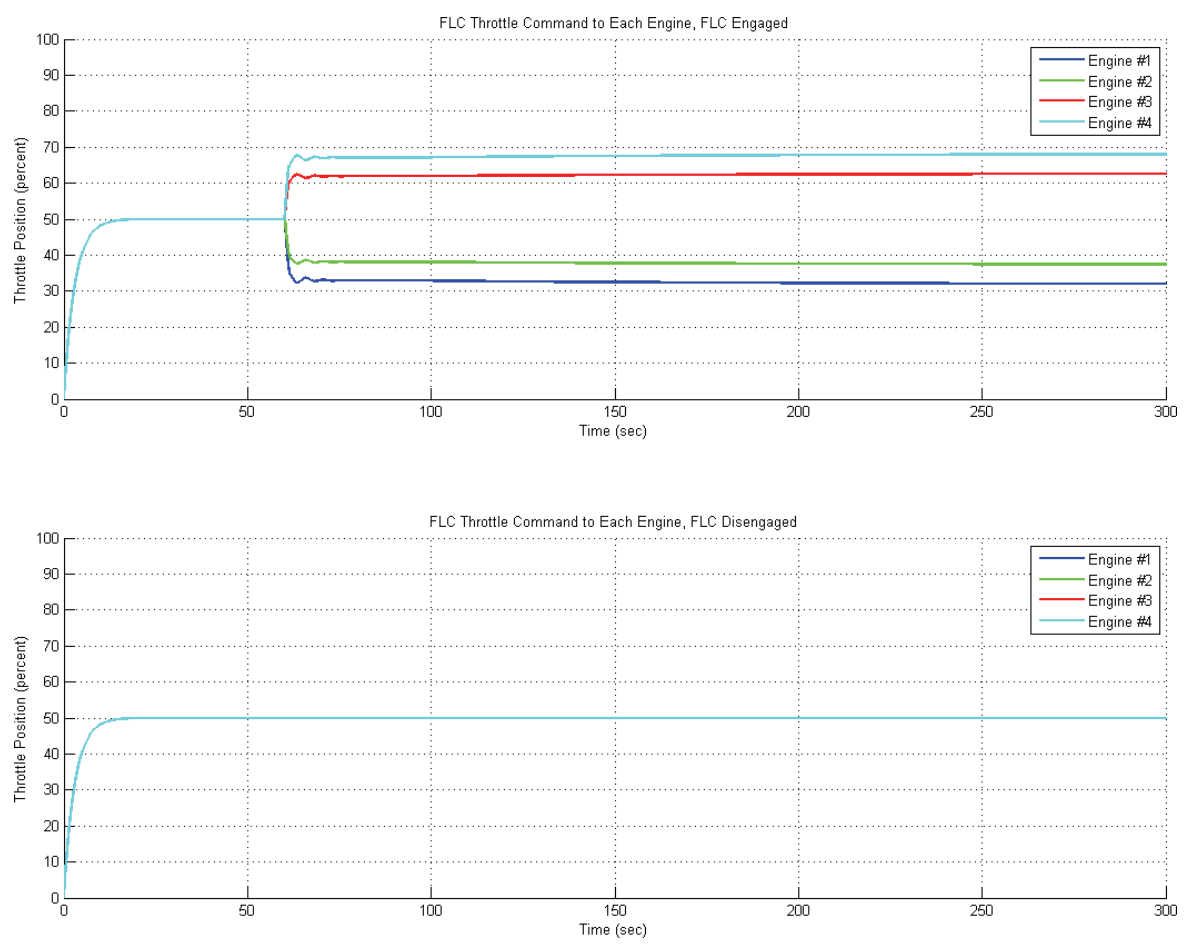

Figure 8-11: Throttle Commands for the +10 Degree Left Aileron Failure Case with the FLC Engaged and Disengaged 

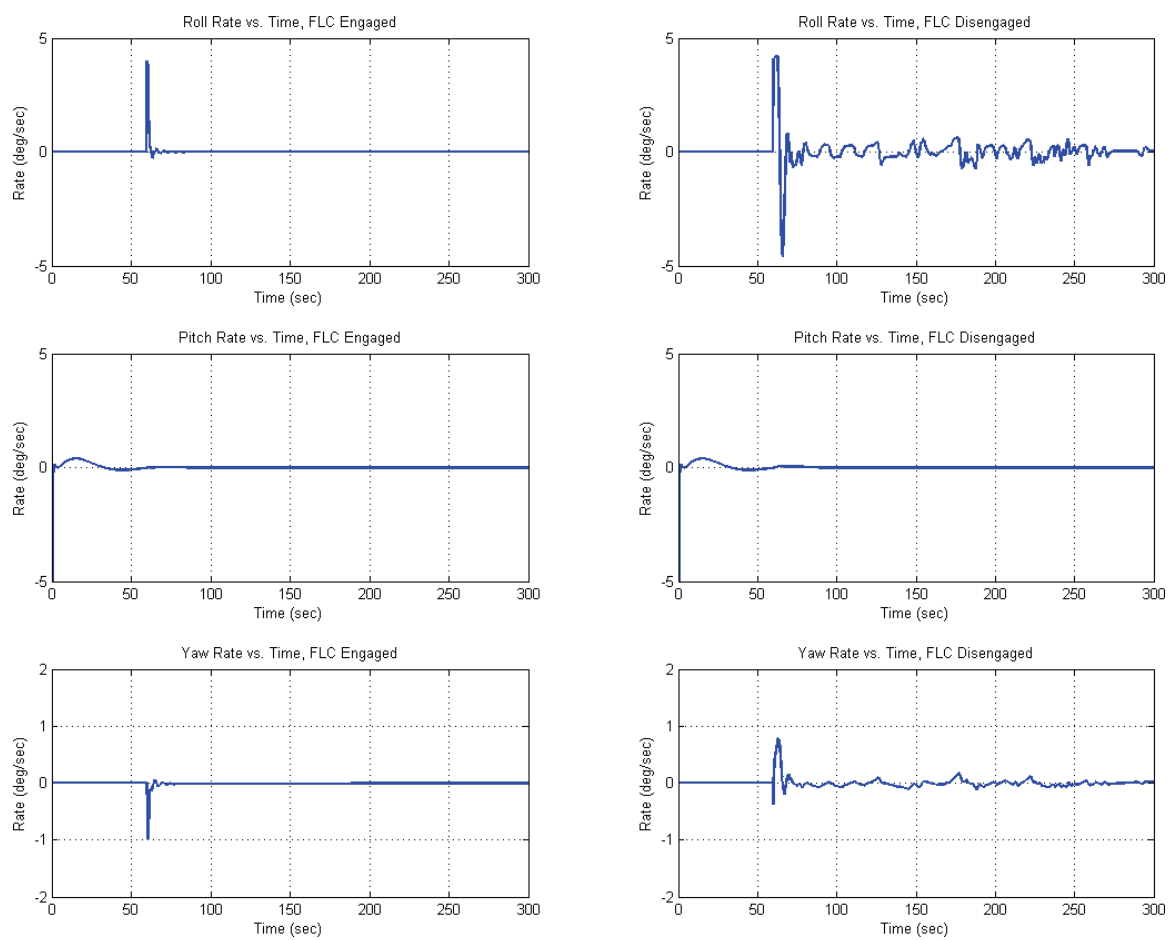

Figure 8-12: Roll, Pitch, and Yaw Rates for the +10 Degree Left Aileron Failure Case with the FLC Engaged and Disengaged
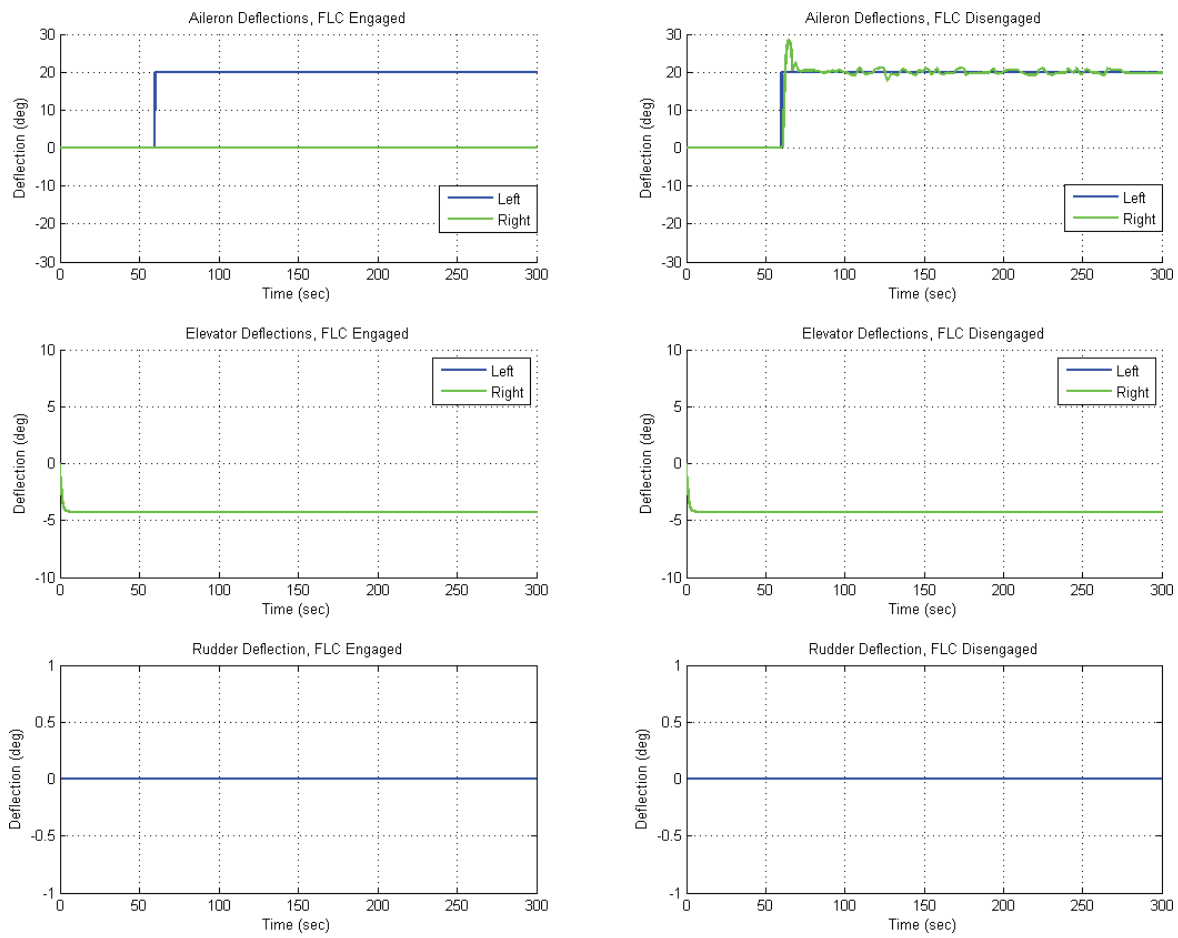

Figure 8-13: Control Surface Deflections for the +20 Degree Left Aileron Failure Case with the FLC Engaged and Disengaged 

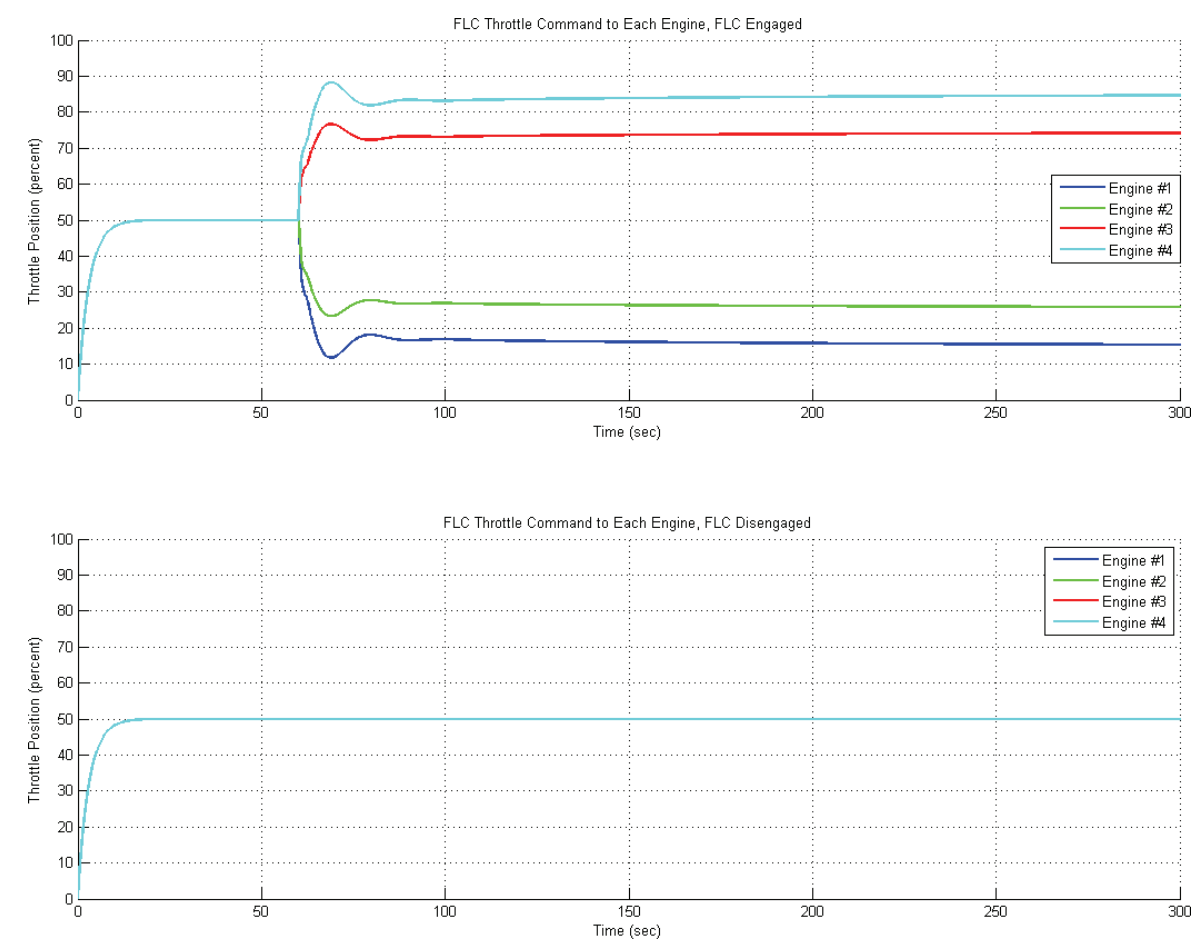

Figure 8-14: Throttle Commands for the +20 Degree Left Aileron Failure Case with the FLC Engaged and Disengaged
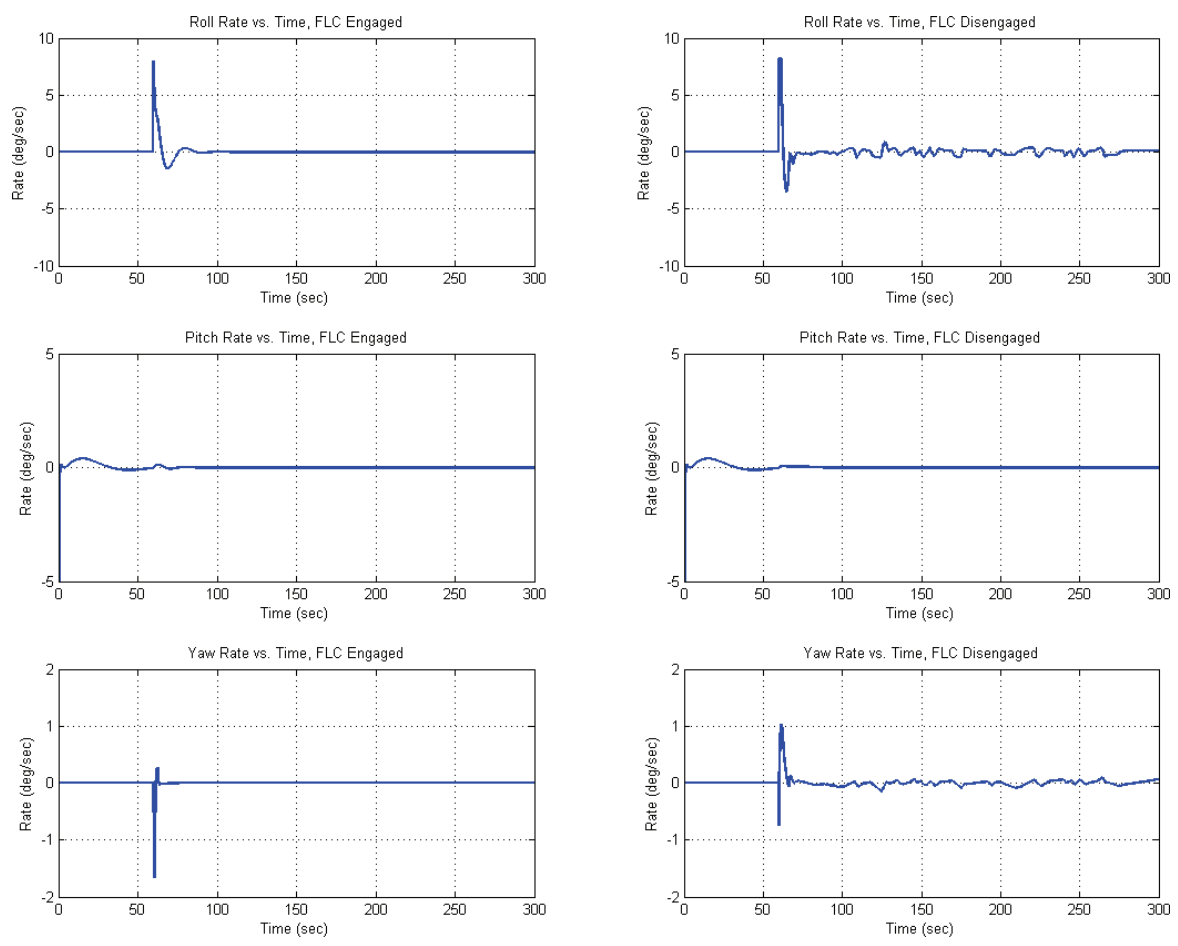

Figure 8-15: Roll, Pitch, and Yaw Rates for the +20 Degree Left Aileron Failure Case with the FLC Engaged and Disengaged 


\subsection{Rudder Failure}

The model was exposed to two levels of rudder actuator failure. Additionally, it was tested at each level of failure with the FLC throttle control engaged and disengaged. The magnitudes of failure considered were stuck rudder actuator at +10 degrees and +15 degrees. During each test, the aircraft was subjected to the indicated failure at the 60 second mark such that there is some nominal flight data before the abnormal condition. The engine locations were assumed symmetrical as in the nominal flight test. Furthermore, differential thrust about the longitudinal plane was utilized.

When the aircraft was exposed to the +10 degree stuck rudder actuator, it was controllable while the FLC was engaged and disengaged. While the controller was engaged, no aileron input was required in order to maintain level flight whereas while the controller was disengaged an average of 12.4 degrees of aileron deflection was necessary to compensate for the rolling moment (Figure 8-16). The FLC throttle commands can be viewed in Figure 8-17. From here it is noticeable that the engine control is nearly saturated but there is still room for pilot throttle control authority. Finally, when examining the roll rate and yaw rate in Figure 8-18 while the controller was engaged and disengaged, the FLC controller dampens out many of the peaks that are notable in the disengaged controller case. Furthermore, when the initial peak from the rudder failure is ignored from the yaw rate as seen in Figure 8-19 it can be seen that the FLC successfully dampens the yaw rate.

When the aircraft was exposed to the +15 degree stuck rudder actuator failure, it was controllable with the FLC engaged and disengaged. This does not indicate that the flight was comfortable or successful. The pilot was required to supply aileron input to maintain level flight while the controller was engaged. The average amount of deflection was less than one degree. 
This is encouraging to the effectiveness for utilizing engine thrust for actuator failure accommodation. When the left aileron was deflected +20 degrees, the FLC was able to accommodate the failure with a margin of control authority in reserve as seen in Figure 8-14. This margin was utilized under the +15 degree rudder actuator failure and the additional aileron input. When the controller was disengaged the average deflection required to counteract the induced roll moment was 19 degrees as seen in Figure 8-20. As seen in Figure 8-21, the throttle input is completely saturated. The roll, pitch, and yaw rates can be seen in Figure 8-22.

Two solutions were examined to increase the effectiveness of the controller. The first solution involves increasing the amount of potential thrust for each engine. By using this method and an equation similar to equation (7.41) but expanded for four engines, the amount of thrust necessary is $113 \%$ of the nominal thrust. To ensure a margin of safety, the amount of potential thrust amount was increased to $125 \%$ of nominal. During the course of the experiment the throttle commands were briefly saturated at their maximum value shortly after the failure was initiated at the 60 second mark. Shortly thereafter, the controller was able to stabilize with the Engine $\# 1$ command leveling out at $112 \%$ and Engine $\# 2$ was below the nominal maximum thrust as viewed in Figure 8-23. As further proof to the effectiveness of this method, Figure 8-24 displays control surface deflections as an indication that no additional pilot input was needed and Figure 8-25 presents the roll, pitch, and yaw rates.

The second solution involved moving the outer engine locations while the inner engines remained fixed and the amount of thrust control to all engines remained equivalent to the nominal conditions test. For the purpose of this research effort, any potential structural issues related to this solution have not been considered. Using equations similar to (7.42), (7.43), and (7.44), the minimum outer engine location necessary for control was found to be $\left[\begin{array}{lll}r_{x} & r_{y} & r_{z}\end{array}\right]=$ 
$\left[\begin{array}{lll}-1.75 & \pm 24.5 & 1.5\end{array}\right]$ meters instead of the nominal location of $\left[\begin{array}{lll}r_{x} & r_{y} & r_{z}\end{array}\right]=\left[\begin{array}{lll}0 & \pm 21.96 & 1.69\end{array}\right]$ meters. During the test a margin was added to the minimum engine location position such that the engine positions were $\left[\begin{array}{lll}r_{x} & r_{y} & r_{z}\end{array}\right]=$

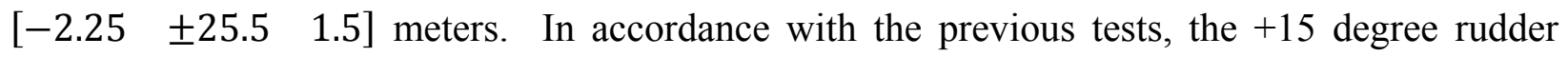
failure was initiated at the 60 second mark. The controller was capable of stabilizing the flight. As seen in Figure 8-26, the throttle control for the outer engines is still completely saturated but the inner engines have margin of control authority that is not evident in the +15 degree rudder actuator failure with the engines in the nominal location (Figure 8-21). If the inner engines were repositioned toward the wing tips in the same manner as the outer engines, more control authority could be obtained for the same amount of engine thrust. Furthermore, pilot input was not necessary as evident by the control surface deflections in Figure 8-27. The roll, pitch, and yaw rates displayed in Figure 8-28 returned to zero after the failure indicating that the aircraft returned back to steady-state flight.
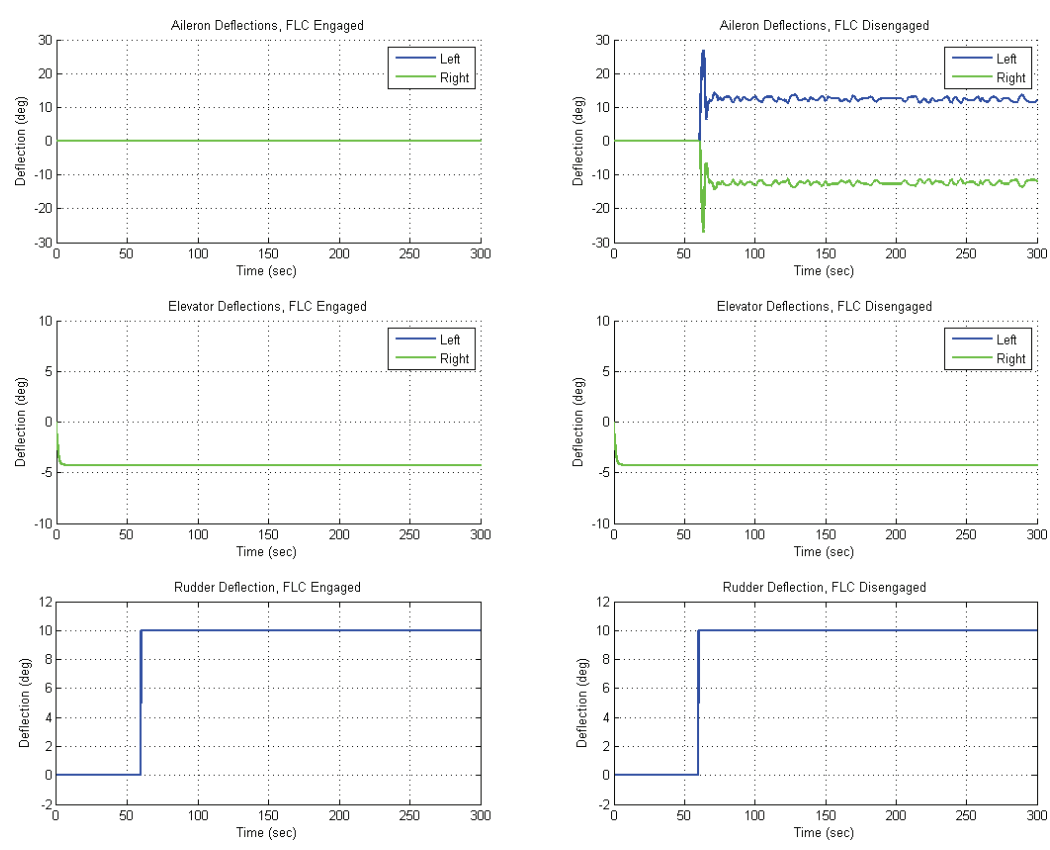

Figure 8-16: Control Surface Deflections for the +10 Degree Rudder Failure Case with the FLC Engaged and Disengaged 

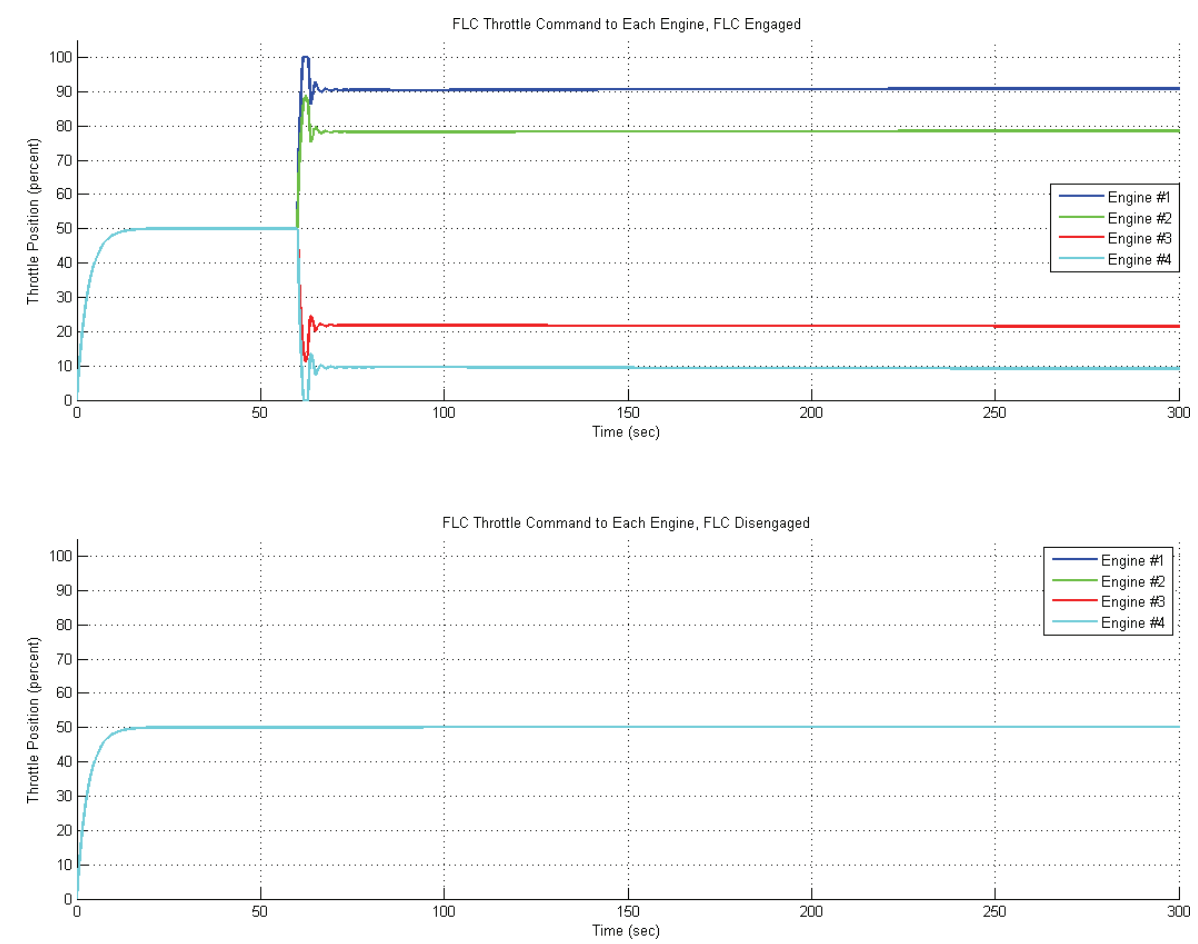

Figure 8-17: Throttle Commands for the +10 Degree Rudder Failure Case with the FLC Engaged and Disengaged
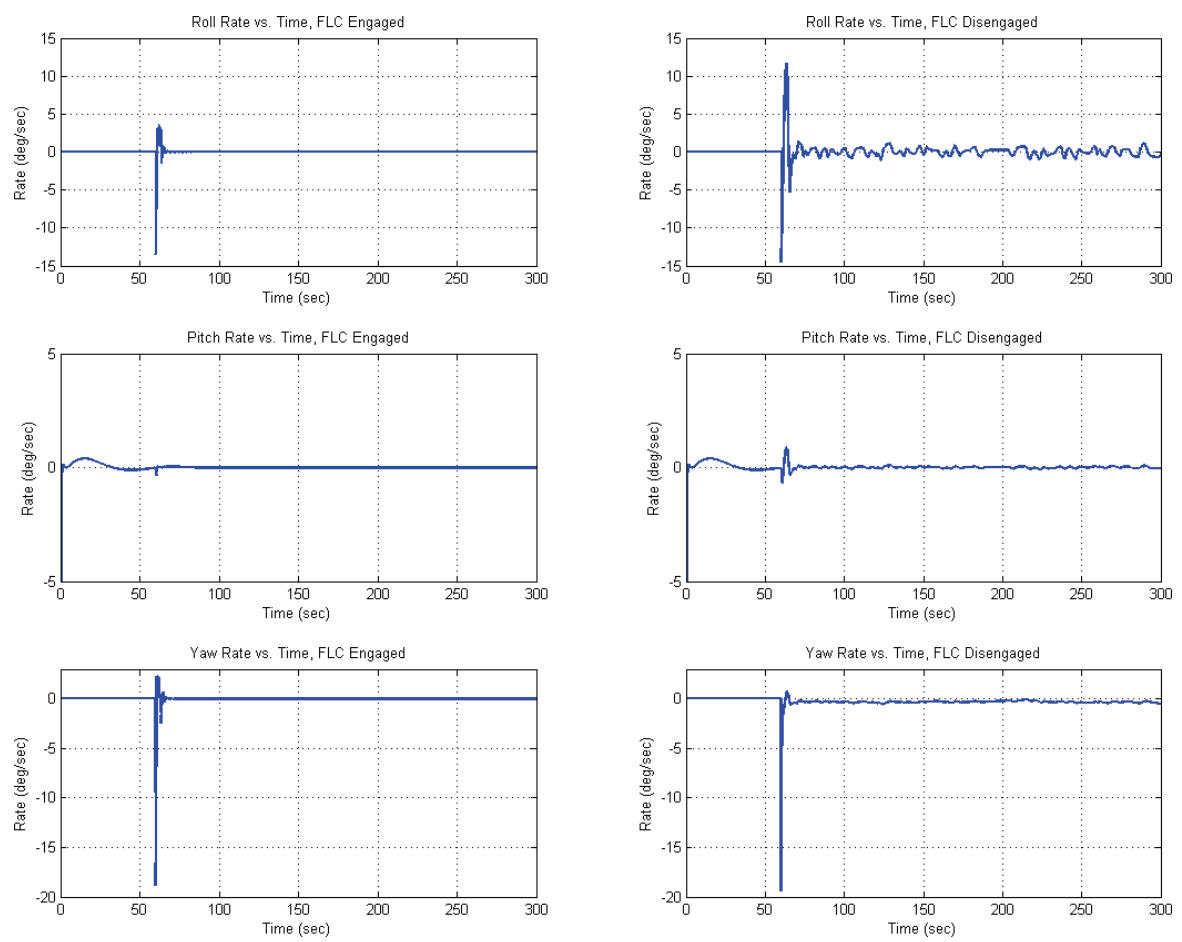

Figure 8-18: Roll, Pitch, and Yaw Rates for the +10 Degree Rudder Failure Case with the FLC Engaged and Disengaged 

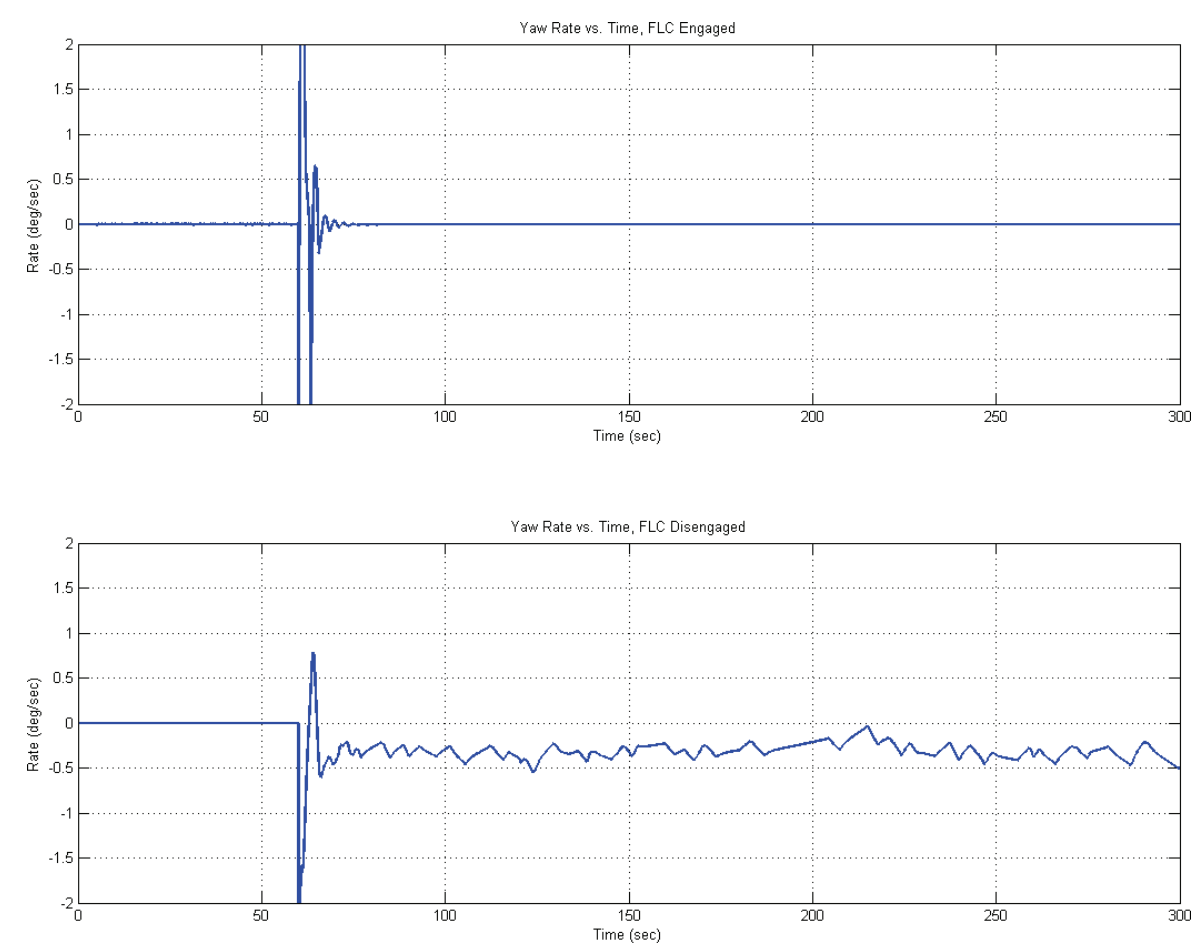

Figure 8-19: Yaw Rate without the Initial Peak for the +10 Degree Rudder Failure Case with the FLC Engaged and Disengaged
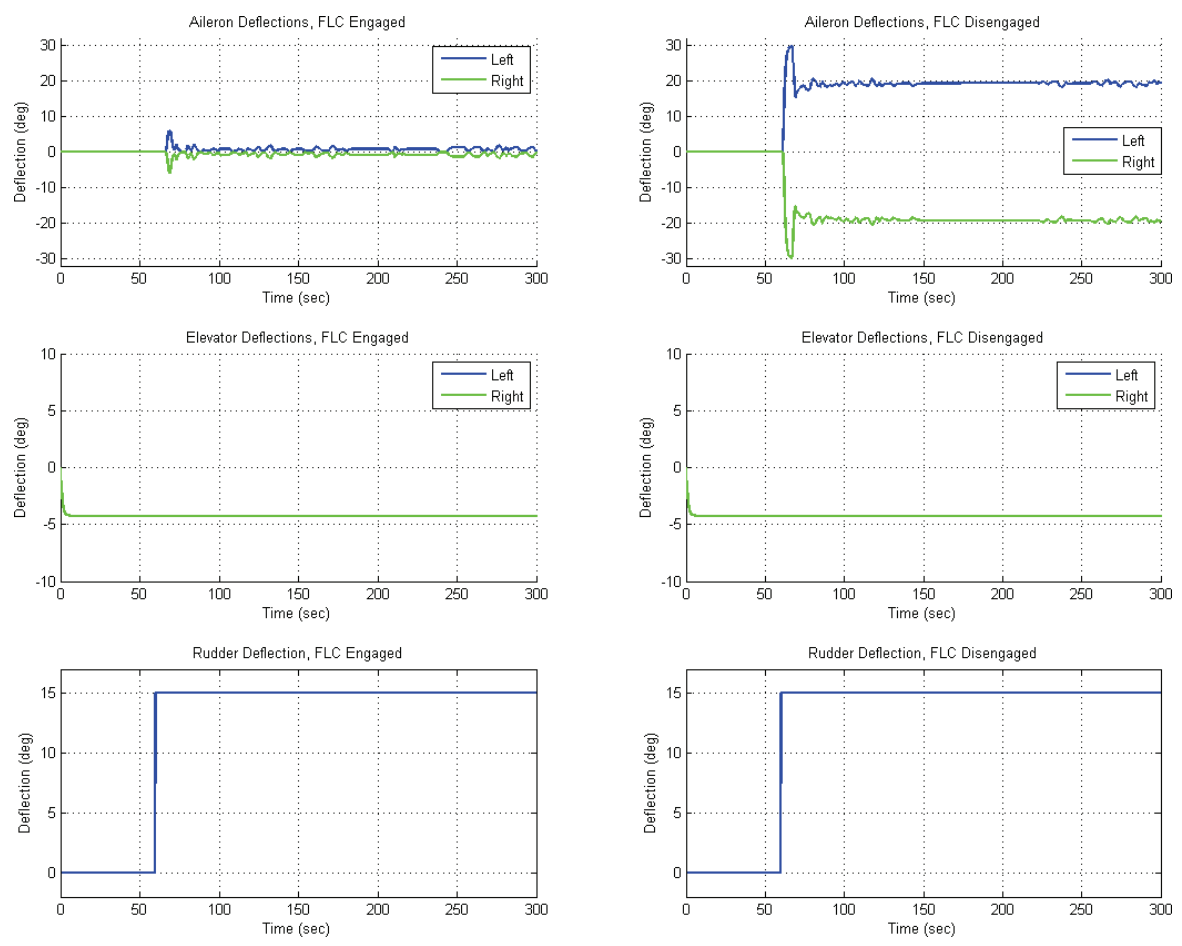

Figure 8-20: Control Surface Deflections for the +15 Degree Rudder Failure Case with the FLC Engaged and Disengaged 

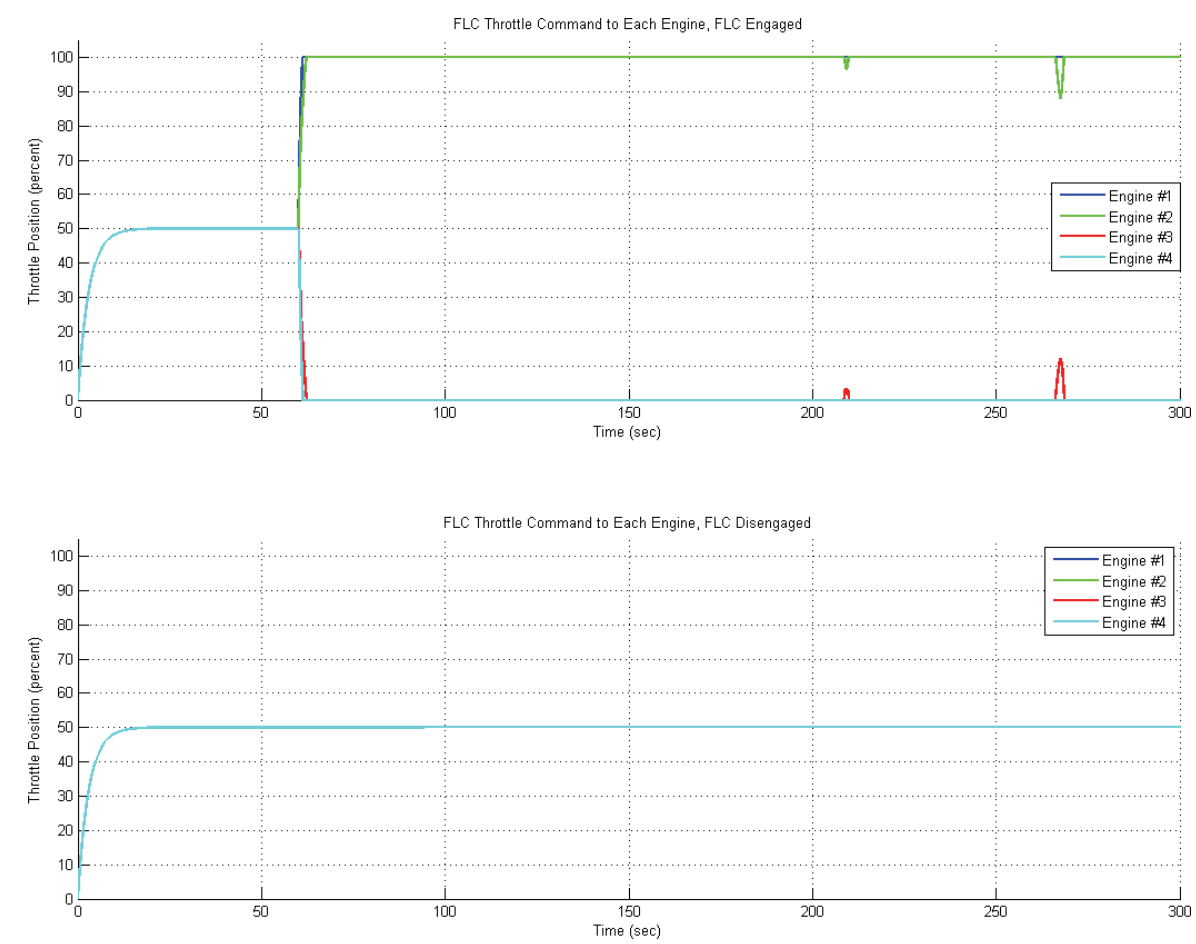

Figure 8-21: Throttle Commands for the +15 Degree Rudder Failure Case with the FLC Engaged and Disengaged
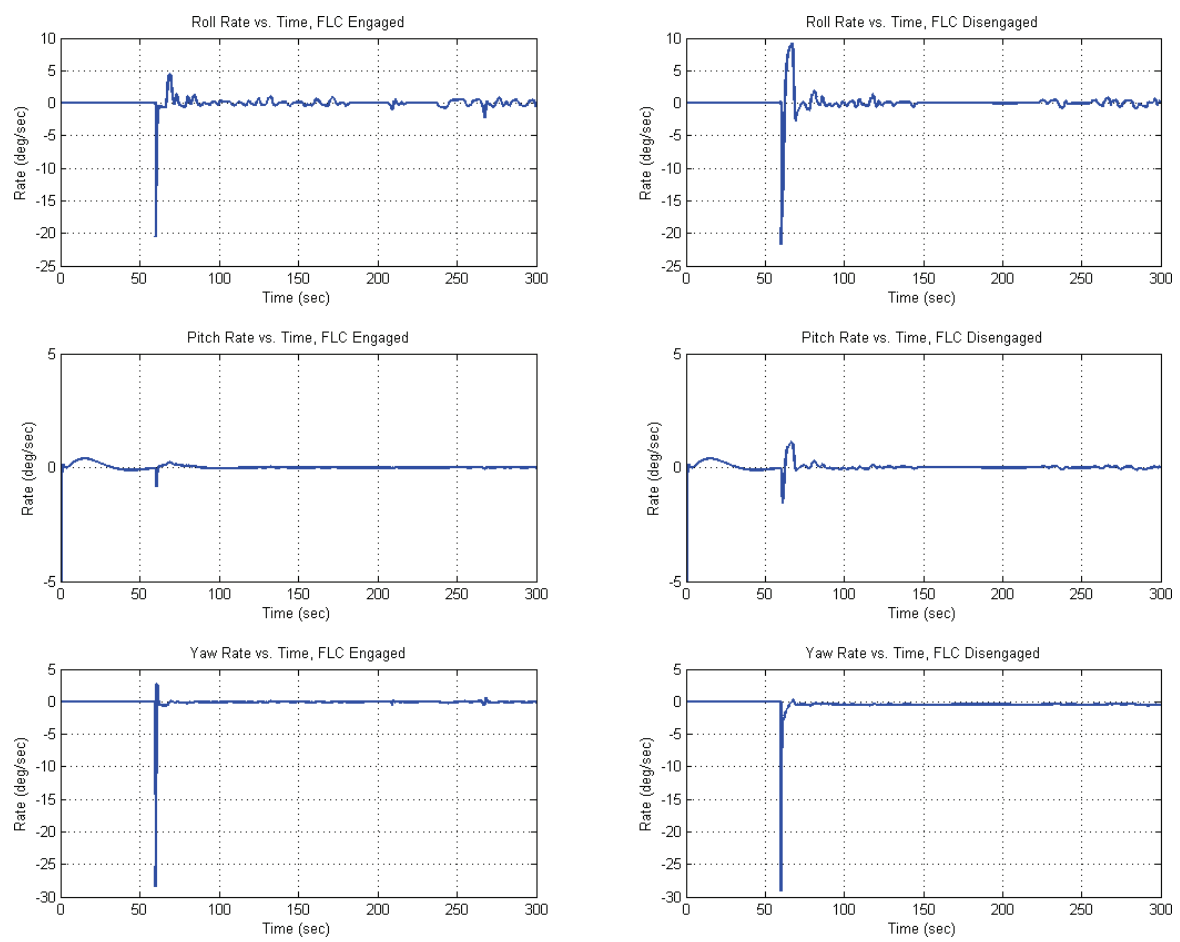

Figure 8-22: Roll, Pitch, and Yaw Rates for the +15 Degree Rudder Failure Case with the FLC Engaged and Disengaged 


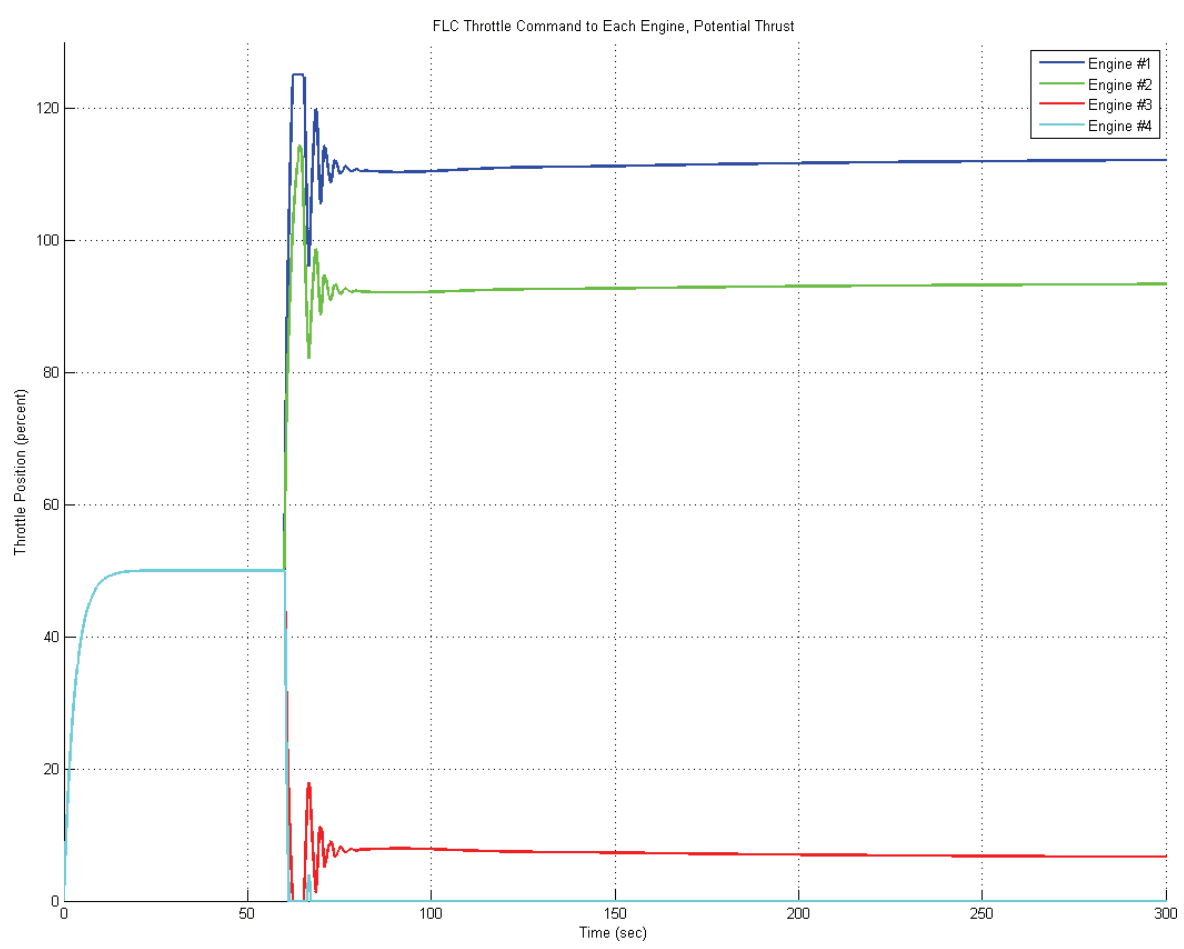

Figure 8-23: Throttle Commands for the +15 Degree Rudder Failure when the Thrust Potential is Increased
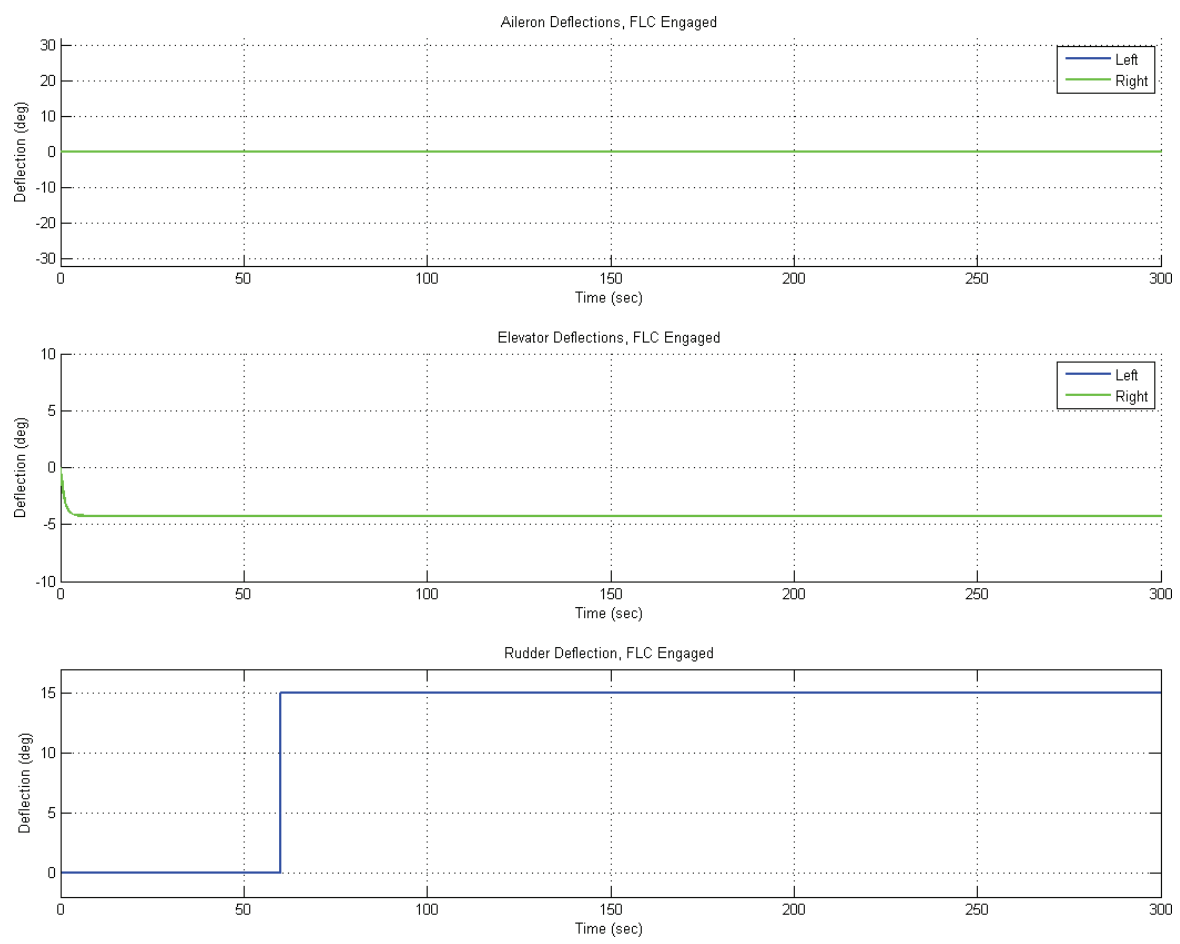

Figure 8-24: Control Surface Deflections for the +15 Degree Rudder Failure when the Thrust Potential is Increased 


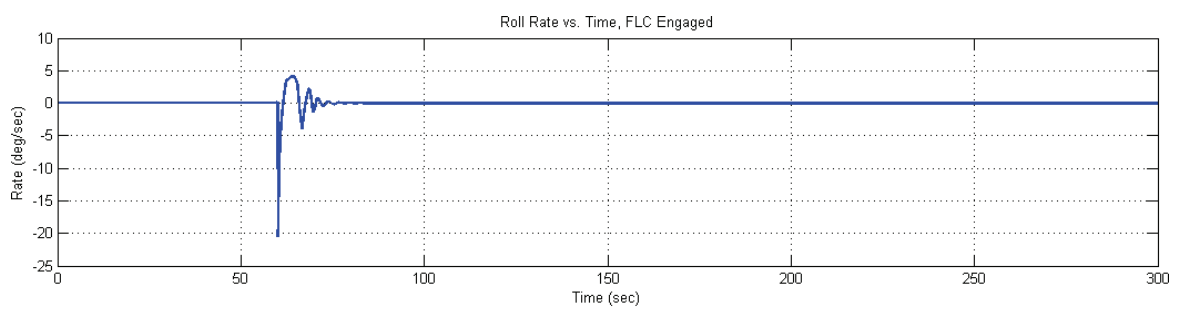

Pitch Rate vs. Time, FLC Engaged
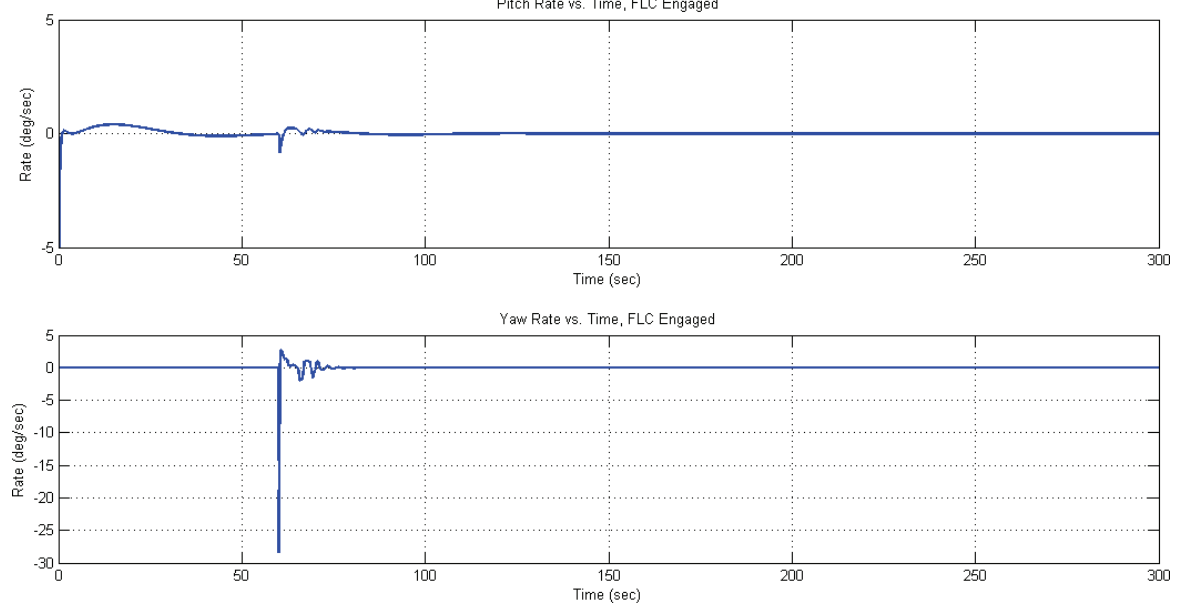

Figure 8-25: Roll, Pitch, and Yaw Rates for the +15 Degree Rudder Failure when the Thrust Potential is Increased

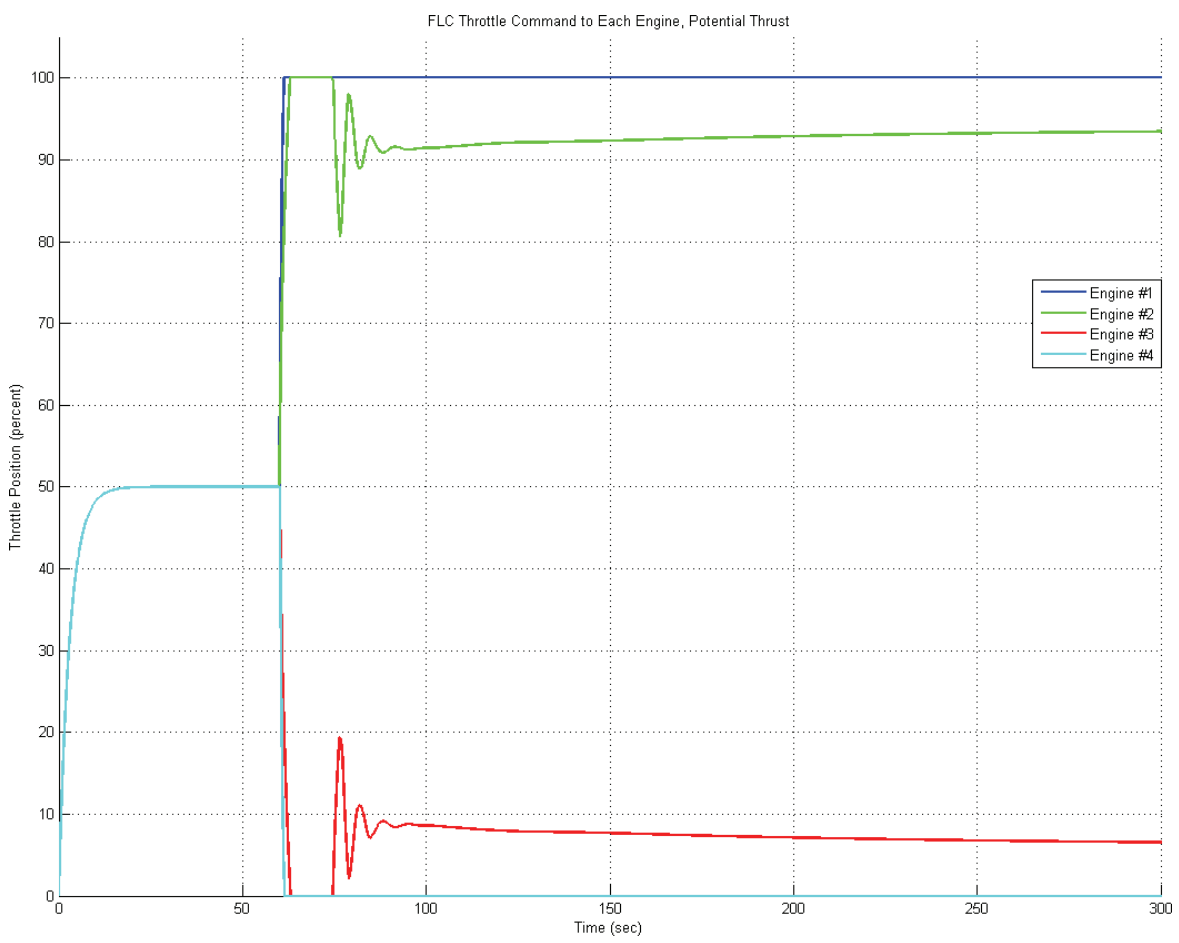

Figure 8-26: Throttle Commands for the +15 Degree Rudder Failure when the Outer Engine Locations are Moved 

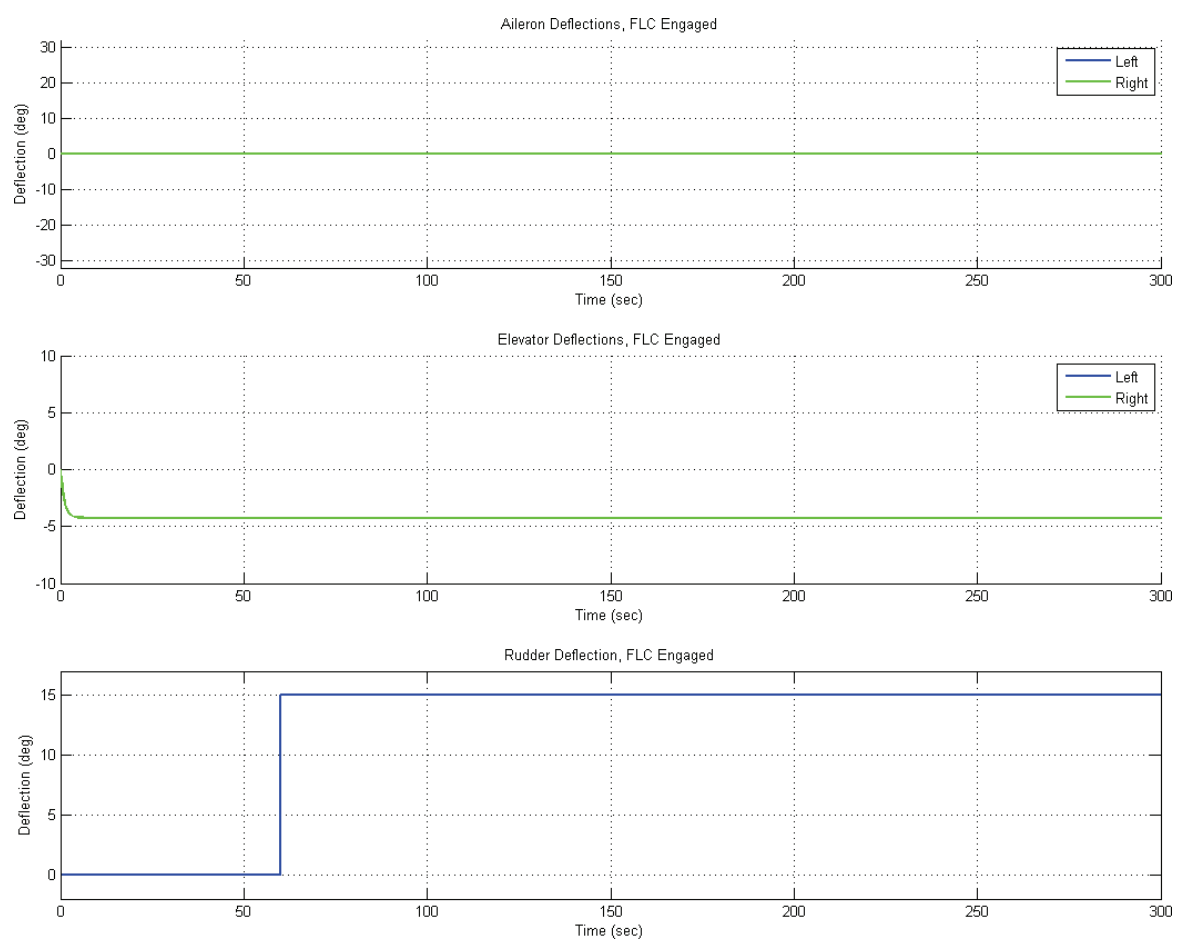

Figure 8-27: Control Surface Deflections for the +15 Degree Rudder Failure when the Outer Engine Locations are Moved
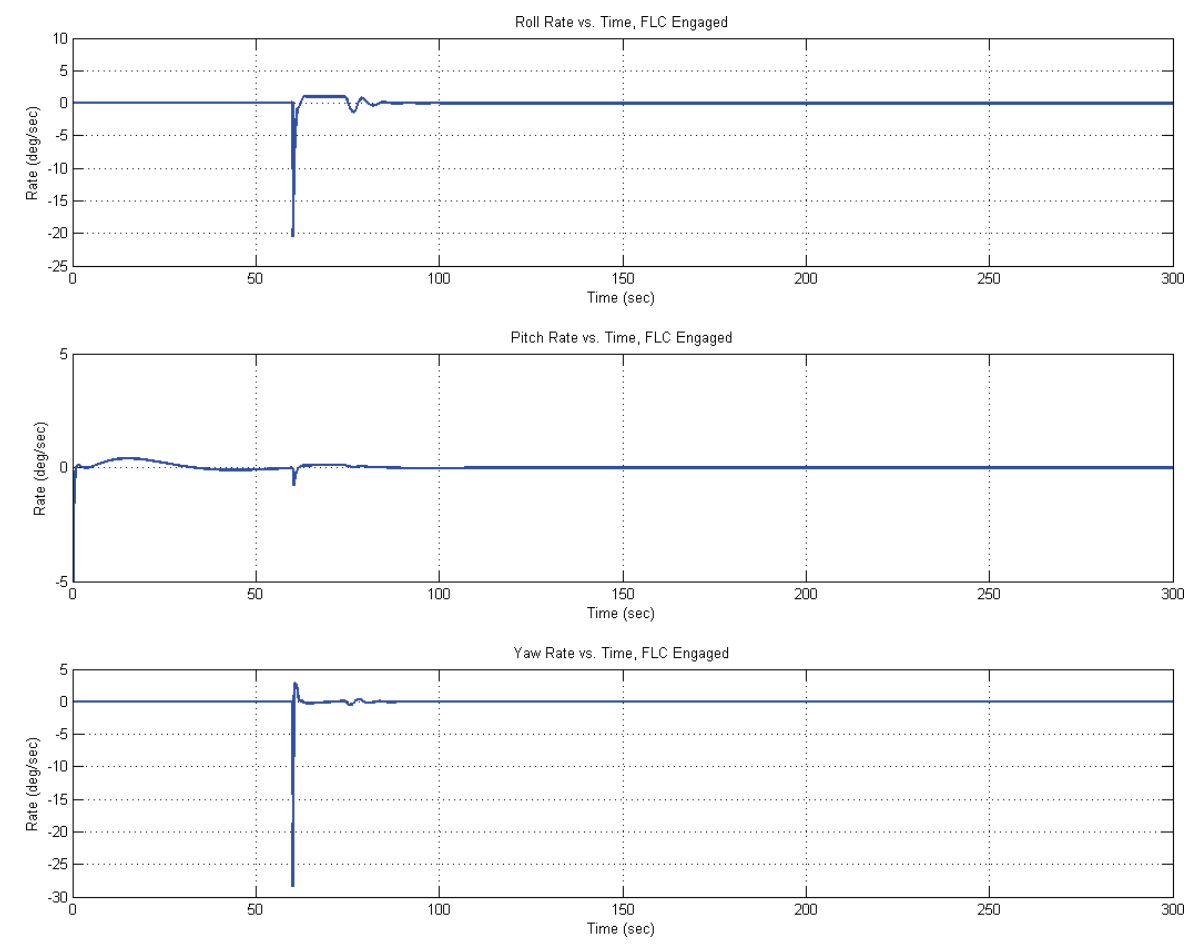

Figure 8-28: Roll, Pitch, and Yaw Rates for the +15 Degree Rudder Failure when the Outer Engine Locations are Moved 


\section{Chapter 9: Conclusions and Recommendations}

A large transport aircraft model based on a Boeing 747-400 was developed as the testing platform for the throttle control work presented here. A FLC was developed for the lateral, longitudinal, and directional components of the aircraft control system. Furthermore, the aircraft model was modified such that it could accept between two and ten engines where the user can specify the exact number and the location of each engine. The FLC automatically adjusts for the number selected.

Real-time simulation flight data was obtained from the large transport aircraft where the pilot attempted to maintain steady level flight for the duration of each test. Each experiment was flown twice: once with the FLC engaged and once with the FLC disengaged. This was completed in order to assess the effectiveness of the throttle FLC. Through the course of these experiments, the FLC was proven to be effective. The controller allowed the pilot to retain more control authority over the aircraft than when it was disengaged.

The aircraft design aspects of the model were tested during the +15 rudder actuator failure scenario. By allowing additional thrust control authority, the design requirements for a suitable engine for the given failure were established. Additionally, by altering the engine locations given the maximum thrust available, a solution was found such that the aircraft was capable of accommodating the given failure. This framework can be used for the evaluation of the failure accommodation potential of any given engine configuration, to determine fixed characteristics of the propulsion system necessary to be able to compensate for actuator failure of a specified severity through differential throttle, and design fault tolerant engine control laws in 
term of engine throttle, translation, and rotation. The effectiveness of the approach was illustrated through an example simulation.

The model allows the effective determination of the maximum failure deflection that can be accommodated by a given configuration, the required engine position for a specified failure magnitude and engine power, and the maximum thrust needed to compensate a certain actuator failure if the location of the propulsion device is fixed.

The FLC developed provides the basic framework to expand the use of engine thrust control for actuator failure accommodation. One way the model could be expanded would be to include active thrust vectoring into the control system. Additionally, the system could include a dynamic engine placement mechanism where the engines would not be fixed in relation to the aircrafts center of gravity. The engines would be allowed to change location in order to counteract an actuator failure without altering the thrust output from the individual engines. Finally, this type of dynamic engine control could be implemented within a different control scheme. For example, WVU has developed an advanced supersonic fighter jet model that utilizes several types of neural networks for flight control and adaptation ${ }^{(39)}$. Currently, these control systems use decoupled control surfaces to collectively counteract an actuator failure. This type of system could be used to initially counteract the failure that the model is subjected to due to its faster response time and then the scheme could differ some or all of the control to the engines through individual engine thrust control allowing for a larger flight envelope to be obtained. 


\section{References}

1. Liu, Y., et al., Adaptive Compensation of Aircraft Actuation Failures Using an Engine

Differential Model. s.1. : IEEE Transactions on Control Systems Technology, 2008. pp. 971-982.

2. Burcham, F. Jr., et al., Development and Flight Test of an Emergency Flight Control System Using Only Engine Thrust on an MD-11 Transport Airplane. Oct. 1997. NASA TP-97-206217.

3. Burcham, F. Jr., et al., Development and Flight Evaluation of an Emergency Digital Flight Control System Using Only Engine Thrust on an F-15 Airplane. 1996. NASA TP-3627.

4. Vincenzi, D. A., et al., Human Factors in Simulation and Training. Boca Raton : Taylor \& Francis Group, LLC, 2009. 13L978-1-4200-7283-9.

5. Kaempffert, W. Dry Shooting for Airplane Gunners. Popular Science Monthly. Jan. 1919, Vol. 94, 1, pp. 13-14.

6. - Flight Simulators Technology Through the Years. Fly Away Simulation. [Online] July 12, 2010. [Cited: Oct. 15, 2011.] http://flyawaysimulation.com/news/4045/flight-simulatortechnology-through-years/.

7. Canadian Aviation Electronics. CAE 7000 Series Level D Full-Flight Simulator. [Online] [Cited: Nov. 2, 2011.] http://www.cae.com/en/sim.products/_pdf/datasheet.cae.7000.series.pdf. 8. - . Review of Aircraft Accident Data. National Transportation Safety Board. [Online] 20072011. [Cited: Sept. 5, 2011.] http://www.ntsb.gov/doclib/reports/2011/ARA1101.pdf. ARA1101.

9. - . The 1997-2010 Nall Reports. Aircraft Owners and Pilots Association Online. [Online] 1997-2010. [Cited: Sept. 5, 2011.] http://www.aopa.org/asf/publications/nall.html. 
10. - . Aviation Safety Network Aviation Safety Database. Aviation Safety Network. [Online]

1943-2011. [Cited: Sept. 5, 2011.] http://aviation-safety.net/database/.

11. - . Accident and Incident Data. Federal Aviation Administration. [Online] 1978-2011.

[Cited: Sept. 5, 2011.] http://www.faa.gov/data_research/accident_incident/.

12. Atkins, E. M., et al., "Solus: An Autonomous Aircraft for Flight Control and Trajectory

Planning Research". s.1. : Proceedings of the 1998 American Control Conference, June 1998. pp. 689-693.

13. Kaneshige, J., Bull, J. and Totah, J. J. Generic Neural Flight Control and Autopilot System. 2000. AIAA 2000-4281.

14. Bodson, M. and Groszkiewicz, J. E. Multivariable Adaptive Algorithms for Reconfigurable Flight Control. s.l. : IEEE Transactions on Control Systems Technology, 1997. pp. 217-229.

15. Wang, Q. and Stengel, R. F. Robust Nonlinear Flight Control of a High-Performance Aircraft. s.1. : IEEE Transactions on Control Systems Technology, 2005. pp. 15-26.

16. Shore, D. and Bodson, M. Flight Testing of a Reconfigurable Control System on an Unmanned Aircraft. s.1. : Proceedings of the 2004 American Control Conference, 2004. pp. $3747-3752$.

17. Perhinschi, M. G., Napolitano, M. R. and Campa, G. "A Simulation Environment for Design and Testing of Aircraft Adaptive Fault-Tolerant Control Systems". Aircraft Engineering and Aerospace Technology: An International Journal. December 2008, Vol. 80, 6, pp. 620-632. 
18. -. Aircraft Accident Report: United Airlines Flight 232, McDonnell Douglas DC-10-10, Sioux Gateway Airport, Sioux City, Iowa, July 19, 1989. s.l. : National Transportation Safety Board, 1990. Tech. Report. NTSB/AAR-90/06.

19. Bodson, M. and Groszkiewicz, J. E. Multivariable Adaptive Algorithms for Reconfigurable Flight Control. Lake Buena Vista, FL : Proceedings of the 33rd Conference on Decision and Control, Dec. 1994. pp. 3330-3335.

20. Kaneshige, J. and Gundy-Burlet, K. Integrated Neural Flight and Propulsion Control System. 2001. AIAA 2001-4386.

21. Kaneshige, J., et al., Propulsion Control with Flight Director Guidace as an Emergency Flight Control System. 1999. AIAA 99-3962.

22. Kaneshige, J., Bull, J. and Totah, J. Generic Neural Flight Control and Autopilot System. 2000. AIAA 2000-4281.

23. Bošković, J. D. and Mehra, R. K. A Multiple Model-based Reconfigurable Flight Control System Design. Tampa, FL : Proceedings of the 37th IEEE Conference on Decision \& Control, 1998.

24. Gopinathan, M., et al., A Multiple Model Predictive Scheme for Fault-Tolerant Flight Control Design. Tampa, FL : Proceedings of the 37th IEEE Conference on Decision \& Control, 1998.

25. Zhang, Y. and Jiang, J. Integrated Active Fault-Tolerant Control Using IMM Approach. s.1. : IEEE Transactions on Aerospace and Electronic Systems, 2001. pp. 1221-1235. 
26. Bošković, J. D. and Mehra, R. K. Stable Multiple Model Adaptive Flight Control for Accommodation of a Large Class of Control Effector Failures. San Diego, CA : Proceedings of the American Control Conference, June 1999.

27. Bošković, J. D. and Mehra, R. An Adaptive Scheme for Compensation of Loss of Effectiveness of Flight Control Effectors. Orlando, FL : Proceedings of the 40th IEEE Conference on Decision and Control, Dec. 2001.

28. Natrajan, A. Consistency Maintenance in Concurrent Representations. 2000. p. 105. Dissertation.

29. Liu, Y., et al., Adaptive Failure Compensation for Aircraft Flight Control Using Engine Differentials: Regulation. Arlington, VA : Infotech@Aerospace Conference, Sept. 2005. AIAA 2005-6996.

30. Pedrycz, W. Fuzzy Control and Fuzzy Systems. s.1. : John Wiley \& Sons, Inc., 1993.

31. Perhinschi, M. G. and Beamer, F. B. Analysis of Aircraft Multiple Engine Configurations for Fault Tolerant Control. Portland, OR : AIAA Guidance, Navigation, and Control Conference, 2011. AIAA-2011-6407.

32. Beamer, F. M., et al., Development of a Simulation Environment to Support Aircraft Health Management Education. Portland, OR : AIAA Atmospheric Flight Mechanics Conference, 2011. AIAA-2011-6614.

33. Rauw, M. O. FDC 1.2 - A SIMULINK Toolbox for Flight Dynamics and Control Analysis. Delft, The Netherlands : Delft University of Technology, 1998. 
34. Basler, M., et al., The FlightGear Manual. [Online] Aug. 15, 2011. [Cited: Aug. 28, 2011.] http://mapserver.flightgear.org/getstart/.

35. Roskam, J. Airplane Flight Dynamics and Automatic Flight Controls. s.1. : Roskam Aviation and Engineering Corporation, 1979.

36. Perhinschi, M. G., et al., "Modeling and Simulation of a Fault Tolerant Control System". International Journal of Modelling and Simulation. January 2006, Vol. 26, 1, pp. 1-10.

37. Chen, G. and Pham, T. Introduction to Fuzzy Sets, Fuzzy logic, and Fuzzy Control Systems. Washington, D.C. : CRC Press, 2001.

38. Buckley, J. and Jowers, L. Monte Carlo Methods in Fuzzy Optimization. s.1. : SpringerVerlag Berlin Heidelberg, 2008.

39. Sagoo, G. K., et al., Evaluation of a Fault-Tolerant Scheme in a Six-Degree-of-Freedom Motion Flight Simulator. s.l. : Journal of Aerospace Computing, Information, and Communication, 2010. pp. 47-67. DOI: 10.2514/1.42299.

40. Antoniewicz, R. F., Duke, E. L. and Patterson, B. P. "User's Manual for Interactive LINEAR, a Fortran Program to Derive Linear Aircraft Models". s.1. : NASA Technical Paper $2835,1988$.

41. Perhinschi, M. G., et al., Development of Fault-Tolerant Flight Control Laws for the WVU YF-22 Model Aircraft. Hilton Head, SC : AIAA Guidance, Navigation, and Control conference and Exibit, 2007. AIAA 2007-6511.

42. Moncayo, H. Y. Immunity-Based Detection, Identification, and Evaluation of Aircraft SubSystem Failures. Morgantown, WV : WVU Dissertation, 2009. 
43. Burken, J. J., et al., Adaptive Control Using Neural Network Augmentation for a Modified F-15 Aircraft. s.1. : Control and Automation, 2006. MED '06. 14th Mediterranean Conference on, 2006. doi: 10.1109/MED.2006.328857.

44. Perhinschi, M. G., Napolitano, M. R. and Campa, G. A Simulation Environment for

Design and Testing of Aircraft Adaptive Fault-Tolerant Control Systems. s.1. : Aircraft

Engineering and Aerospace Technology: An International Journal, 2008. 


\section{Appendix A: Analysis of Engine Compensation for a Tri-Engine Aircraft Configuration}

The complete governing equation from equation (3.17) and (6.1) is:

$$
\begin{aligned}
\sum_{i=1}^{n}\left\{\left[\begin{array}{ccc}
0 & -r_{i z} & r_{i y} \\
r_{i z} & 0 & -r_{i x} \\
-r_{i y} & r_{i x} & 0
\end{array}\right]_{B}\left[\begin{array}{c}
\cos \psi_{i} \cos \theta_{i} \\
\sin \psi_{i} \cos \theta_{i} \\
\sin \theta_{i}
\end{array}\right]_{B} \Delta T_{i}\right\} \\
=\sum_{j=1}^{m}\left\{\left[\begin{array}{l}
\left(M_{x \delta_{j}}\right)_{N} \\
\left(M_{y \delta_{j}}\right)_{N} \\
\left(M_{z \delta_{j}}\right)_{N}
\end{array}\right]_{B} \delta_{j C}-\left[\begin{array}{l}
\left(M_{x \delta_{j}}\right)_{F} \\
\left(M_{y \delta_{j}}\right)_{F} \\
\left(M_{z \delta_{j}}\right)_{F}
\end{array}\right]_{B} \delta_{j F}\right\}
\end{aligned}
$$

If the aerodynamic control surface $\mathrm{j}$ is stuck at a deflection $\delta_{j F}$. This means that the aerodynamic characteristics of the control surface are not altered and the following holds true:

$$
\frac{d \vec{M}_{N j}}{d \delta_{j}}=\frac{d \vec{M}_{F j}}{d \delta_{j}}
$$

and the moment due to the failure is:

$$
\vec{M}_{F j}=\frac{d \vec{M}_{N j}}{d \delta_{j}}\left(\delta_{j C}-\delta_{j F}\right)
$$

Equation (7.1) becomes:

$$
\begin{array}{r}
\sum_{i=1}^{n}\left\{\left[\begin{array}{ccc}
0 & -r_{i z} & r_{i y} \\
r_{i z} & 0 & -r_{i x} \\
-r_{i y} & r_{i x} & 0
\end{array}\right]_{B}\left[\begin{array}{c}
\cos \psi_{i} \cos \theta_{i} \\
\sin \psi_{i} \cos \theta_{i} \\
\sin \theta_{i}
\end{array}\right]_{B} \Delta T_{i}\right\} \\
=\sum_{j=1}^{m}\left\{\left(\delta_{j C}-\delta_{j F}\right)\left[\begin{array}{l}
\left(M_{x \delta_{j}}\right)_{N} \\
\left(M_{y \delta_{j}}\right)_{N} \\
\left(M_{z \delta_{j}}\right)_{N}
\end{array}\right]_{B}\right\}
\end{array}
$$


For the following discussion it is assumed that the aircraft is equipped with three identical engines $(n=3$, left $=L$, center $=C$, right $=R)$ located symmetrically such that:

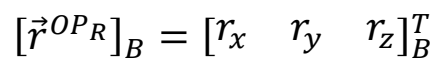

$$
\begin{aligned}
& {\left[\vec{r}^{O P_{C}}\right]_{B}=\left[\begin{array}{lll}
r_{x C} & 0 & r_{z C}
\end{array}\right]_{B}^{T}}
\end{aligned}
$$

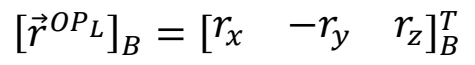

$$
\begin{aligned}
& {\left[\Delta \vec{T}_{R}\right]_{B}=\left[\begin{array}{c}
\Delta T_{x R} \\
\Delta T_{y R} \\
\Delta T_{z R}
\end{array}\right]_{B}=L_{B T_{R}}\left[\Delta \vec{T}_{R}\right]_{T_{R}}=\left[\begin{array}{c}
\cos \psi_{R} \cos \theta_{R} \\
\sin \psi_{R} \cos \theta_{R} \\
\sin \theta_{R}
\end{array}\right]_{B} \Delta T_{R}=\left[\begin{array}{c}
\varepsilon_{1 R} \\
\varepsilon_{2 R} \\
\varepsilon_{3 R}
\end{array}\right]_{B} \Delta T_{R}} \\
& {\left[\Delta \vec{T}_{C}\right]_{B}=\left[\begin{array}{c}
\Delta T_{x C} \\
\Delta T_{y C} \\
\Delta T_{z C}
\end{array}\right]_{B}=L_{B T_{C}}\left[\Delta \vec{T}_{C}\right]_{T_{C}}=\left[\begin{array}{c}
\cos \psi_{C} \cos \theta_{C} \\
\sin \psi_{C} \cos \theta_{C} \\
\sin \theta_{C}
\end{array}\right]_{B} \Delta T_{C}=\left[\begin{array}{c}
\varepsilon_{1 C} \\
\varepsilon_{2 C} \\
\varepsilon_{3 C}
\end{array}\right]_{B} \Delta T_{C}} \\
& {\left[\Delta \vec{T}_{L}\right]_{B}=\left[\begin{array}{c}
\Delta T_{x L} \\
\Delta T_{y L} \\
\Delta T_{z L}
\end{array}\right]_{B}=L_{B T_{L}}\left[\Delta \vec{T}_{L}\right]_{T_{L}}=\left[\begin{array}{c}
\cos \psi_{L} \cos \theta_{L} \\
\sin \psi_{L} \cos \theta_{L} \\
\sin \theta_{L}
\end{array}\right]_{B} \Delta T_{L}=\left[\begin{array}{c}
\varepsilon_{1 L} \\
\varepsilon_{2 L} \\
\varepsilon_{3 L}
\end{array}\right]_{B} \Delta T_{L}}
\end{aligned}
$$

\section{A.1 Elevator Failure}

If the assumption is made that that the left elevator is locked at a deflection $\delta_{e L F}$, then the failure will affect the pitching moment and the rolling moment. It is assumed that the effects on the yawing moment are negligible. Under these conditions and omitting the subscript $\mathrm{N}$ for simplicity, equation (A.4) becomes: 


$$
\begin{gathered}
{\left[\begin{array}{ccc}
0 & -r_{z} & r_{y} \\
r_{z} & 0 & -r_{x} \\
-r_{y} & r_{x} & 0
\end{array}\right]_{B}\left[\begin{array}{c}
\Delta T_{x R} \\
\Delta T_{y R} \\
\Delta T_{z R}
\end{array}\right]_{B}+\left[\begin{array}{ccc}
0 & -r_{z C} & 0 \\
r_{z C} & 0 & -r_{x C} \\
0 & r_{x C} & 0
\end{array}\right]_{B}\left[\begin{array}{c}
\Delta T_{x C} \\
\Delta T_{y C} \\
\Delta T_{z C}
\end{array}\right]_{B}} \\
+\left[\begin{array}{ccc}
0 & -r_{z} & -r_{y} \\
r_{z} & 0 & -r_{x} \\
r_{y} & r_{x} & 0
\end{array}\right]_{B}\left[\begin{array}{c}
\Delta T_{x L} \\
\Delta T_{y L} \\
\Delta T_{z L}
\end{array}\right]_{B}=\left(\delta_{e L C}-\delta_{e L F}\right)\left[\begin{array}{l}
M_{x \delta_{e L}} \\
M_{y \delta_{e L}} \\
M_{z \delta_{e L}}
\end{array}\right]_{B}
\end{gathered}
$$

Furthermore:

$$
\begin{gathered}
{\left[\begin{array}{c}
-r_{z}\left(\Delta T_{y R}+\Delta T_{y L}\right)+r_{y}\left(\Delta T_{z R}-\Delta T_{z L}\right)-r_{z C} \Delta T_{y C} \\
r_{z}\left(\Delta T_{x R}+\Delta T_{x L}\right)-r_{x}\left(\Delta T_{z R}+\Delta T_{z L}\right)+r_{z C} \Delta T_{x C}-r_{x C} \Delta T_{z C} \\
-r_{y}\left(\Delta T_{x R}-\Delta T_{x L}\right)+r_{x}\left(\Delta T_{y R}+\Delta T_{y L}\right)+r_{x C} \Delta T_{y C}
\end{array}\right]_{B}} \\
=\left(\delta_{e L C}-\delta_{e L F}\right)\left[\begin{array}{c}
M_{x \delta_{e L}} \\
M_{y \delta_{e L}} \\
0
\end{array}\right]_{B}
\end{gathered}
$$

or using equations (A.8), (A.9), and (A.10), equation (A.12) becomes:

$$
\begin{gathered}
{\left[\begin{array}{c}
-r_{z}\left(\Delta T_{R} \varepsilon_{2 R}+\Delta T_{L} \varepsilon_{2 L}\right)+r_{y}\left(\Delta T_{R} \varepsilon_{3 R}-\Delta T_{L} \varepsilon_{3 L}\right)-r_{z C} \Delta T_{C} \varepsilon_{3 C} \\
r_{z}\left(\Delta T_{R} \varepsilon_{1 R}+\Delta T_{L} \varepsilon_{1 L}\right)-r_{x}\left(\Delta T_{R} \varepsilon_{3 R}+\Delta T_{L} \varepsilon_{3 L}\right)+r_{z C} \Delta T_{C} \varepsilon_{1 C}-r_{x C} \Delta T_{C} \varepsilon_{3 C} \\
-r_{y}\left(\Delta T_{R} \varepsilon_{1 R}-\Delta T_{L} \varepsilon_{1 L}\right)+r_{x}\left(\Delta T_{R} \varepsilon_{2 R}+\Delta T_{L} \varepsilon_{2 L}\right)+r_{x C} \Delta T_{C} \varepsilon_{2 C}
\end{array}\right]_{B}} \\
=\left(\delta_{e L C}-\delta_{e L F}\right)\left[\begin{array}{c}
M_{x \delta_{e L}} \\
M_{y \delta_{e L}} \\
0
\end{array}\right]_{B}
\end{gathered}
$$

This is a system of three equations and fourteen unknowns $\left(r_{x}, r_{y}, r_{z}, r_{x C}, r_{z C}, \Delta T_{R}, \Delta T_{C}, \Delta T_{L}, \theta_{R}\right.$, $\psi_{R}, \theta_{C}, \psi_{C}, \theta_{L}$, and $\left.\psi_{L}\right)$. If it is assumed that changing the direction of the thrust vector is not used for control. Therefore, the engine Euler angles are held constant and respectively equal in magnitude and symmetric for the left and right engine. As a consequence:

$$
\varepsilon_{1 R}=\varepsilon_{1 L}=\varepsilon_{1}, \varepsilon_{2 R}=\varepsilon_{2 L}=\varepsilon_{2}, \varepsilon_{3 R}=\varepsilon_{3 L}=\varepsilon_{3}
$$


and the number of unknowns - or variables that can potentially be used for control - is reduced to eight $\left(r_{x}, r_{y}, r_{z}, r_{x C}, r_{z C}, \Delta T_{R}, \Delta T_{C}\right.$, and $\left.\Delta T_{L}\right)$ :

$$
\begin{gathered}
{\left[\begin{array}{c}
-r_{z}\left(\Delta T_{R}+\Delta T_{L}\right) \varepsilon_{2}+r_{y}\left(\Delta T_{R}-\Delta T_{L}\right) \varepsilon_{3}-r_{z C} \Delta T_{C} \varepsilon_{3 C} \\
r_{z}\left(\Delta T_{R}+\Delta T_{L}\right) \varepsilon_{1}-r_{x}\left(\Delta T_{R}+\Delta T_{L}\right) \varepsilon_{3}+r_{z C} \Delta T_{C} \varepsilon_{1 C}-r_{x C} \Delta T_{C} \varepsilon_{3 C} \\
-r_{y}\left(\Delta T_{R}-\Delta T_{L}\right) \varepsilon_{1}+r_{x}\left(\Delta T_{R}-\Delta T_{L}\right) \varepsilon_{2}+r_{x C} \Delta T_{C} \varepsilon_{2 C}
\end{array}\right]_{B}} \\
=\left(\delta_{e L C}-\delta_{e L F}\right)\left[\begin{array}{c}
M_{x \delta_{e L}} \\
M_{y \delta_{e L}} \\
0
\end{array}\right]_{B}
\end{gathered}
$$

If the axes of the engines are parallel to the aircraft longitudinal axis then $\varepsilon_{1}=\varepsilon_{1 C}=1, \varepsilon_{2}=$ $\varepsilon_{2 C}=\varepsilon_{3}=\varepsilon_{3 C}=0$, and the compensating engine moment is:

$$
\left[\sum_{i=1}^{3} \vec{M}_{e i}\right]_{B}=\left[\begin{array}{c}
0 \\
r_{z}\left(\Delta T_{R}+\Delta T_{L}\right)+r_{z C} \Delta T_{C} \\
-r_{y}\left(\Delta T_{R}-\Delta T_{L}\right)
\end{array}\right]_{B}
$$

In this case, the rolling effects of the failure cannot be compensated and an undesirable yawing moment is produced unless $\Delta T_{R}=\Delta T_{L}=\Delta T$. The pitching effects of the failure can be compensated if the engines are located off the body-axes horizontal plane $\left(r_{z} \neq 0\right)$. If this is true, then:

$$
2 r_{z} \Delta T+r_{z C} \Delta T_{C}=\left(\delta_{e L C}-\delta_{e L F}\right)
$$

To compensate for the rolling effects of the failure, $\varepsilon_{2} \neq 0$ and/or $\varepsilon_{3} \neq 0$ as well as $\varepsilon_{2 C} \neq 0$ and/or $\varepsilon_{3 C} \neq 0$. Collective thrust $\left(\Delta T_{R}=\Delta T_{C}=\Delta T_{L}=\Delta T\right)$ or differential thrust $\left(\Delta T_{R} \neq \Delta T_{C} \neq \Delta T_{L}\right)$ can be used. For the collective thrust case, equation (A.15) becomes:

$$
\left[\begin{array}{c}
-2 r_{z} \Delta T \varepsilon_{2}-r_{z C} \Delta T \varepsilon_{3 C} \\
2 r_{z} \Delta T \varepsilon_{1}-2 r_{x} \Delta T \varepsilon_{3}+r_{z C} \Delta T \varepsilon_{1 C}-r_{x C} \Delta T \varepsilon_{3 C} \\
0
\end{array}\right]_{B}=\left(\delta_{e L C}-\delta_{e L F}\right)\left[\begin{array}{c}
M_{x \delta_{e L}} \\
M_{y \delta_{e L}} \\
0
\end{array}\right]_{B}
$$


The following conditions result as necessary for rolling compensation:

$$
r_{z} \neq 0, r_{z C} \neq 0, \psi_{R}=\psi_{L} \neq 0, \psi_{C} \neq 0, \theta_{R}=\theta_{L} \neq \frac{\pi}{2} \text {, and } \theta_{C} \neq \frac{\pi}{2}
$$

Assuming that the devices considered are the actual aircraft main propulsion system, then $\left|\psi_{R}\right|=\left|\psi_{L}\right| \neq \frac{\pi}{2}, \quad\left|\psi_{C}\right| \neq \frac{\pi}{2}, \quad \varepsilon_{1} \neq 0$, and $\varepsilon_{1 C} \neq 0$. Pitching compensation capabilities are maintained if:

$$
\begin{gathered}
\theta_{R}=\theta_{C}=\theta_{L}=0 \text { or } r_{x}=r_{x C}=0 \Rightarrow \\
2 r_{x} \Delta T \varepsilon_{1}+r_{z C} \Delta T \varepsilon_{1 C}=\left(\delta_{e L C}-\delta_{e L F}\right) M_{y \delta_{e L}}
\end{gathered}
$$

or:

$$
\begin{gathered}
2 r_{z} \varepsilon_{1}+r_{z C} \varepsilon_{1 C} \neq 2 r_{x} \varepsilon_{3}+r_{x C} \varepsilon_{3 C} \quad \Rightarrow \\
\left(2 r_{z} \varepsilon_{1}-2 r_{x} \varepsilon_{3}+r_{z C} \varepsilon_{1 C}-r_{x C} \varepsilon_{3 C}\right) \Delta T=\left(\delta_{e L C}-\delta_{e L F}\right) M_{y \delta_{e L}}
\end{gathered}
$$

For the differential thrust case, considering that $\varepsilon_{1} \neq 0$ and $\varepsilon_{1 C} \neq 0$ for the actual main aircraft propulsion system, the yawing effects are avoided if:

$$
r_{y} \varepsilon_{1}=r_{x} \varepsilon_{2}+r_{x C} \varepsilon_{2 C}
$$

Assuming that the differential thrust control is symmetric, that is $\Delta T_{R}=-\Delta T_{L}=\Delta T$, then engine compensating moment becomes:

$$
\left[\sum_{i=1}^{3} \vec{M}_{e i}\right]_{B}=\left[\begin{array}{c}
2 r_{y} \Delta T \varepsilon_{3}-r_{z C} \Delta T_{C} \varepsilon_{3 C} \\
r_{z C} \Delta T_{C} \varepsilon_{1 C}-r_{x C} \Delta T_{C} \varepsilon_{3 C} \\
r_{x C} \Delta T_{C} \varepsilon_{2 C}
\end{array}\right]_{B}
$$

In order to maintain pitching moment compensation capabilities, $\Delta T_{R} \neq \Delta T_{C} \neq \Delta T_{L}$ and equation (A.15) becomes: 


$$
\begin{gathered}
{\left[\begin{array}{c}
-r_{z}\left(\Delta T_{R}+\Delta T_{L}\right) \varepsilon_{2}+r_{y}\left(\Delta T_{R}-\Delta T_{L}\right) \varepsilon_{3}-r_{z C} \Delta T_{C} \varepsilon_{3 C} \\
r_{z}\left(\Delta T_{R}+\Delta T_{L}\right) \varepsilon_{1}-r_{x}\left(\Delta T_{R}+\Delta T_{L}\right) \varepsilon_{3}+r_{z C} \Delta T_{C} \varepsilon_{1 C}-r_{x C} \Delta T_{C} \varepsilon_{3 C}
\end{array}\right]_{B}} \\
0 \\
=\left(\delta_{e L C}-\delta_{e L F}\right)\left[\begin{array}{c}
M_{x \delta_{e L}} \\
M_{y \delta_{e L}} \\
0
\end{array}\right]_{B}
\end{gathered}
$$

The additional conditions for both rolling and pitching compensation result in:

$$
\begin{gathered}
r_{z}\left(\Delta T_{R}+\Delta T_{L}\right) \varepsilon_{2}+r_{z C} \Delta T_{C} \varepsilon_{3 C} \neq r_{y}\left(\Delta T_{R}-\Delta T_{L}\right) \varepsilon_{3} \\
r_{z}\left(\Delta T_{R}+\Delta T_{L}\right) \varepsilon_{1}+r_{z C} \Delta T_{C} \varepsilon_{1 C} \neq r_{x}\left(\Delta T_{R}+\Delta T_{L}\right) \varepsilon_{3}+r_{x C} \Delta T_{C} \varepsilon_{3 C}
\end{gathered}
$$

If the location and orientation of the three thrust vectors are fixed, then equation (A.24) can be used to solve for $\Delta T_{R}$, and $\Delta T_{L}$ while $\Delta T_{C}$ is controlled by the pilot throttle and is not available for alternative control. Assuming that equation (A.25) and equation (A.26) are valid, this yields:

$$
\begin{array}{r}
\Delta T_{R}=\frac{1}{2}\left(\frac{\left(\delta_{e L C}-\delta_{e L F}\right) M_{y \delta_{e L}}-r_{z C} \Delta T_{C} \varepsilon_{1 C}+r_{x C} \Delta T_{C} \varepsilon_{3 C}}{r_{z} \varepsilon_{1}-r_{x} \varepsilon_{3}}\right. \\
\left.-\frac{\left(\delta_{e L C}-\delta_{e L F}\right) M_{x \delta_{e L}}+r_{z C} \Delta T_{C} \varepsilon_{3 C}}{r_{z} \varepsilon_{2}-r_{y} \varepsilon_{3}}\right) \\
\Delta T_{L}=\frac{1}{2}\left(\frac{\left(\delta_{e L C}-\delta_{e L F}\right) M_{y \delta_{e L}}-r_{z C} \Delta T_{C} \varepsilon_{1 C}+r_{x C} \Delta T_{C} \varepsilon_{3 C}}{r_{z} \varepsilon_{1}-r_{x} \varepsilon_{3}}\right. \\
\left.+\frac{\left(\delta_{e L C}-\delta_{e L F}\right) M_{x \delta_{e L}}+r_{z C} \Delta T_{C} \varepsilon_{3 C}}{r_{z} \varepsilon_{2}-r_{y} \varepsilon_{3}}\right)
\end{array}
$$

\section{A.2 Aileron Failure}

If it is assumed that the left aileron is locked at a deflection $\delta_{a L F}$. The failure will affect primarily the rolling moment and to a lesser extend the yawing moment, while it is assumed that 
the effects on the pitching moments are negligible. Following the same procedure as for the elevator, an equation similar to equation (A.13) is obtained:

$$
\begin{gathered}
{\left[\begin{array}{c}
-r_{z}\left(\Delta T_{R} \varepsilon_{2 R}+\Delta T_{L} \varepsilon_{2 L}\right)+r_{y}\left(\Delta T_{R} \varepsilon_{3 R}-\Delta T_{L} \varepsilon_{3 L}\right)-r_{z C} \Delta T_{C} \varepsilon_{3 C} \\
r_{z}\left(\Delta T_{R} \varepsilon_{1 R}+\Delta T_{L} \varepsilon_{1 L}\right)-r_{x}\left(\Delta T_{R} \varepsilon_{3 R}+\Delta T_{L} \varepsilon_{3 L}\right)+r_{z C} \Delta T_{C} \varepsilon_{1 C}-r_{x C} \Delta T_{C} \varepsilon_{3 C} \\
-r_{y}\left(\Delta T_{R} \varepsilon_{1 R}-\Delta T_{L} \varepsilon_{1 L}\right)+r_{x}\left(\Delta T_{R} \varepsilon_{2 R}+\Delta T_{L} \varepsilon_{2 L}\right)+r_{x C} \Delta T_{C} \varepsilon_{2 C}
\end{array}\right]_{B}} \\
=\left(\delta_{a L C}-\delta_{a L F}\right)\left[\begin{array}{c}
M_{x \delta_{a L}} \\
0 \\
M_{z \delta_{a L}}
\end{array}\right]_{B}
\end{gathered}
$$

Assuming again that changing the direction of the thrust vectors is not used for control and considering equation (A.14):

$$
\begin{gathered}
{\left[\begin{array}{c}
-r_{z}\left(\Delta T_{R}+\Delta T_{L}\right) \varepsilon_{2}+r_{y}\left(\Delta T_{R}-\Delta T_{L}\right) \varepsilon_{3}-r_{z C} \Delta T_{C} \varepsilon_{3 C} \\
r_{z}\left(\Delta T_{R}+\Delta T_{L}\right) \varepsilon_{1}-r_{x}\left(\Delta T_{R}+\Delta T_{L}\right) \varepsilon_{3}+r_{z C} \Delta T_{C} \varepsilon_{1 C}-r_{x C} \Delta T_{C} \varepsilon_{3 C} \\
-r_{y}\left(\Delta T_{R}-\Delta T_{L}\right) \varepsilon_{1}+r_{x}\left(\Delta T_{R}-\Delta T_{L}\right) \varepsilon_{2}+r_{x C} \Delta T_{C} \varepsilon_{2 C}
\end{array}\right]_{B}} \\
=\left(\delta_{a L C}-\delta_{a L F}\right)\left[\begin{array}{c}
M_{x \delta_{a L}} \\
0 \\
M_{z \delta_{a L}}
\end{array}\right]_{B}
\end{gathered}
$$

Since a compensatory rolling moment must be produced, differential vertical and/or lateral thrust components are necessary. If it is assumed that differential symmetric thrust is used $\left(\Delta T_{R}=\right.$ $\left.-\Delta T_{L}=\Delta T\right)$ and $\Delta T_{C}$ is not used for control then:

$$
\left[\begin{array}{c}
2 r_{y} \Delta T \varepsilon_{3} \\
0 \\
-2 r_{y} \Delta T \varepsilon_{1}+2 r_{x} \Delta T \varepsilon_{2}
\end{array}\right]_{B}=\left(\delta_{a L C}-\delta_{a L F}\right)\left[\begin{array}{c}
M_{x \delta_{a L}} \\
0 \\
M_{z \delta_{a L}}
\end{array}\right]_{B}
$$

The additional conditions for rolling moment compensation are:

$$
\theta_{R}=\theta_{L} \neq 0 \text { and } r_{y} \neq 0
$$

Furthermore, yawing compensation is provided if: 


$$
r_{y} \varepsilon_{1} \neq r_{x} \varepsilon_{2}
$$

If the location and orientation of the engine thrust vectors are fixed, then equation (A.31) can be used to solve for $\Delta T$. Assuming that equation (A.32) and equation (A.33) are valid, this yields:

$$
\Delta T=\frac{\left(\delta_{a L C}-\delta_{a L F}\right) M_{x \delta_{a L}}}{2 r_{y} \varepsilon_{3}}
$$

or:

$$
\Delta T=\frac{\left(\delta_{a L C}-\delta_{a L F}\right) M_{z \delta_{a L}}}{2 r_{x} \varepsilon_{2}-2 r_{y} \varepsilon_{1}}
$$

\section{A.3 Rudder Failure}

Assume that the rudder is locked at a deflection $\delta_{r F}$. The failure will affect primarily the yawing and to some extent the rolling moment, while it is assumed that the effects on the pitching moment are negligible. Following the same procedure as for the elevator failure, an equation similar to equation (A.13) is obtained:

$$
\begin{gathered}
{\left[\begin{array}{c}
-r_{z}\left(\Delta T_{R} \varepsilon_{2 R}+\Delta T_{L} \varepsilon_{2 L}\right)+r_{y}\left(\Delta T_{R} \varepsilon_{3 R}-\Delta T_{L} \varepsilon_{3 L}\right)-r_{z C} \Delta T_{C} \varepsilon_{3 C} \\
r_{z}\left(\Delta T_{R} \varepsilon_{1 R}+\Delta T_{L} \varepsilon_{1 L}\right)-r_{x}\left(\Delta T_{R} \varepsilon_{3 R}+\Delta T_{L} \varepsilon_{3 L}\right)+r_{z C} \Delta T_{C} \varepsilon_{1 C}-r_{x C} \Delta T_{C} \varepsilon_{3 C} \\
-r_{y}\left(\Delta T_{R} \varepsilon_{1 R}-\Delta T_{L} \varepsilon_{1 L}\right)+r_{x}\left(\Delta T_{R} \varepsilon_{2 R}+\Delta T_{L} \varepsilon_{2 L}\right)+r_{x C} \Delta T_{C} \varepsilon_{2 C}
\end{array}\right]_{B}} \\
=\left(\delta_{r C}-\delta_{r F}\right)\left[\begin{array}{c}
M_{x \delta_{r}} \\
0 \\
M_{z \delta_{r}}
\end{array}\right]_{B}
\end{gathered}
$$

Assuming again that changing the direction of the thrust vector is not used for control and considering equation (A.14): 


$$
\begin{gathered}
{\left[\begin{array}{c}
-r_{z}\left(\Delta T_{R}+\Delta T_{L}\right) \varepsilon_{2}+r_{y}\left(\Delta T_{R}-\Delta T_{L}\right) \varepsilon_{3}-r_{z C} \Delta T_{C} \varepsilon_{3 C} \\
r_{z}\left(\Delta T_{R}+\Delta T_{L}\right) \varepsilon_{1}-r_{x}\left(\Delta T_{R}+\Delta T_{L}\right) \varepsilon_{3}+r_{z C} \Delta T_{C} \varepsilon_{1 C}-r_{x C} \Delta T_{C} \varepsilon_{3 C} \\
-r_{y}\left(\Delta T_{R}-\Delta T_{L}\right) \varepsilon_{1}+r_{x}\left(\Delta T_{R}+\Delta T_{L}\right) \varepsilon_{2}+r_{x C} \Delta T_{C} \varepsilon_{2 C}
\end{array}\right]_{B}} \\
=\left(\delta_{r C}-\delta_{r F}\right)\left[\begin{array}{c}
M_{x \delta_{r}} \\
0 \\
M_{z \delta_{r}}
\end{array}\right]_{B}
\end{gathered}
$$

Since a compensatory yawing moment must be produced primarily, differential longitudinal thrust components are sufficient provided the distance from the engine to the vertical plane of symmetry is large enough. If differential symmetric thrust $\left(\Delta T_{R}=-\Delta T_{L}=\Delta T\right)$ is used and $\Delta T_{C}$ is not used for control then:

$$
\left[\begin{array}{c}
2 r_{y} \Delta T \varepsilon_{3} \\
0 \\
-2\left(r_{y} \varepsilon_{1}-r_{x} \varepsilon_{2}\right) \Delta T
\end{array}\right]_{B}=\left(\delta_{r C}-\delta_{r F}\right)\left[\begin{array}{c}
M_{x \delta_{r}} \\
0 \\
M_{z \delta_{r}}
\end{array}\right]_{B}
$$

Equation (A.38) shows that if the location and the orientation of the two thrust vectors are fixed then differential thrust commands can be determined in order to produce compensation of the yawing moment. The necessary thrust is:

$$
\Delta T=\frac{\left(\delta_{r C}-\delta_{r F}\right) M_{z \delta_{r}}}{-2\left(r_{y} \varepsilon_{1}-r_{x} \varepsilon_{2}\right)}
$$

However, it might be necessary to compensate for the residual moment on the lateral channel by using the aileron.

If the engine thrust differential is known as well as the moments produced by the failed rudder and assuming that there is no change in the thrust vector, then equation (A.37) can be used to determine the engine positions for the left and right engines. Solving for $r_{x}, r_{y}$, and $r_{z}$ yields: 


$$
\begin{gathered}
r_{x}=\frac{\left(\delta_{r C}-\delta_{r F}\right)\left(M_{x \delta_{r}} \varepsilon_{1}+M_{z \delta_{r}} \varepsilon_{3}\right)+\Delta T_{C} r_{x C}\left(\varepsilon_{1} \varepsilon_{3 C}-\varepsilon_{2 C} \varepsilon_{3}\right)}{2 \varepsilon_{2} \varepsilon_{3} \Delta T_{L}} \\
+\frac{\Delta T_{C} \varepsilon_{2}\left(\Delta T_{L}-\Delta T_{R}\right)\left(\varepsilon_{1 C} r_{z C}-\varepsilon_{3} r_{x C}\right)}{2 \varepsilon_{2} \varepsilon_{3} \Delta T_{L}\left(\Delta T_{L}+\Delta T_{R}\right)} \\
r_{y}=\frac{\left(\Delta T_{L}+\Delta T_{R}\right)\left(-\left(\delta_{r C}-\delta_{r F}\right) M_{x \delta_{r}} \varepsilon_{1}-\Delta T_{C} \varepsilon_{1} \varepsilon_{3} r_{x C}\right)}{2 \varepsilon_{1} \varepsilon_{3} \Delta T_{L}\left(\Delta T_{L}-\Delta T_{R}\right)} \\
+\frac{\left(\delta_{r C}-\delta_{r F}\right) M_{z \delta_{r}} \varepsilon_{3}-\Delta T_{C}\left(\varepsilon_{1 C} \varepsilon_{2} r_{z C}-r_{x C}\left(\varepsilon_{2} \varepsilon_{3 C}-\varepsilon_{2 C} \varepsilon_{3}\right)\right)}{2 \varepsilon_{1} \varepsilon_{3} \Delta T_{L}}
\end{gathered}
$$

$r_{z}$

$=\frac{\left(\delta_{r C}-\delta_{r F}\right)\left(M_{x \delta_{r}} \varepsilon_{1}+M_{z \delta_{r}} \varepsilon_{3}\right)+\Delta T_{C}\left(r_{x C}\left(\varepsilon_{3 C}\left(\varepsilon_{1}+\varepsilon_{2}\right)-\varepsilon_{2 C} \varepsilon_{3}\right)-\varepsilon_{1 C} \varepsilon_{2} r_{z C}\right)}{2 \varepsilon_{1} \varepsilon_{2} \Delta T_{L}}$ 


\section{Appendix B: Simulation Environment User Manual}

Start MATLAB 2010a. Once the program is ready for use, change the current directory to the current directory for the WVU B747 Simulation (example in Figure B-1).

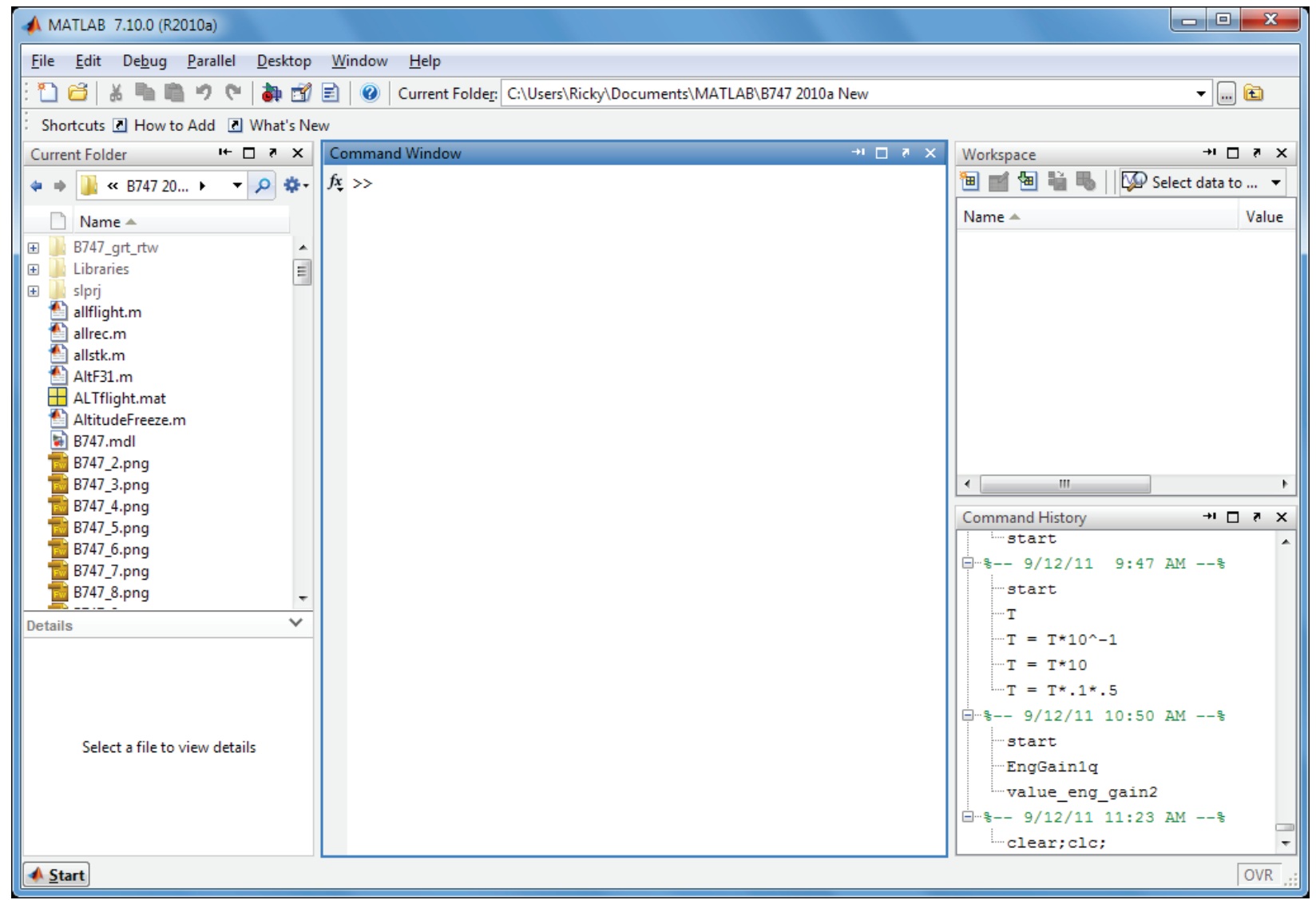

Figure B-0-1: MATLAB Command Window with the Current Directory Set

Type "start" in the command window and press enter (Figure B-2). This will open the West Virginia University Boeing 747 Simulation main menu (Figure B-3). 


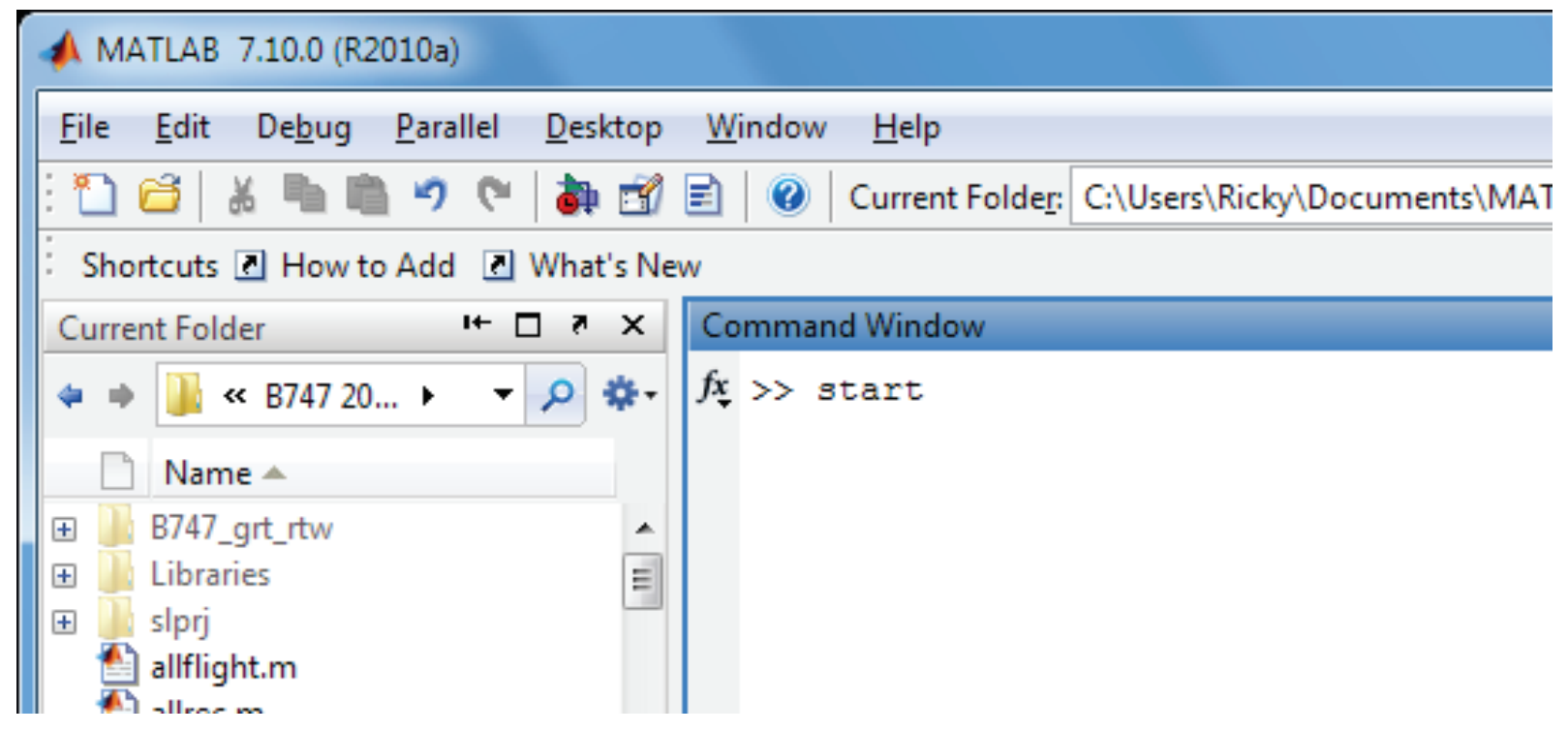

Figure B-0-2: Opening the WVU Boeing 747 Simulation Menu

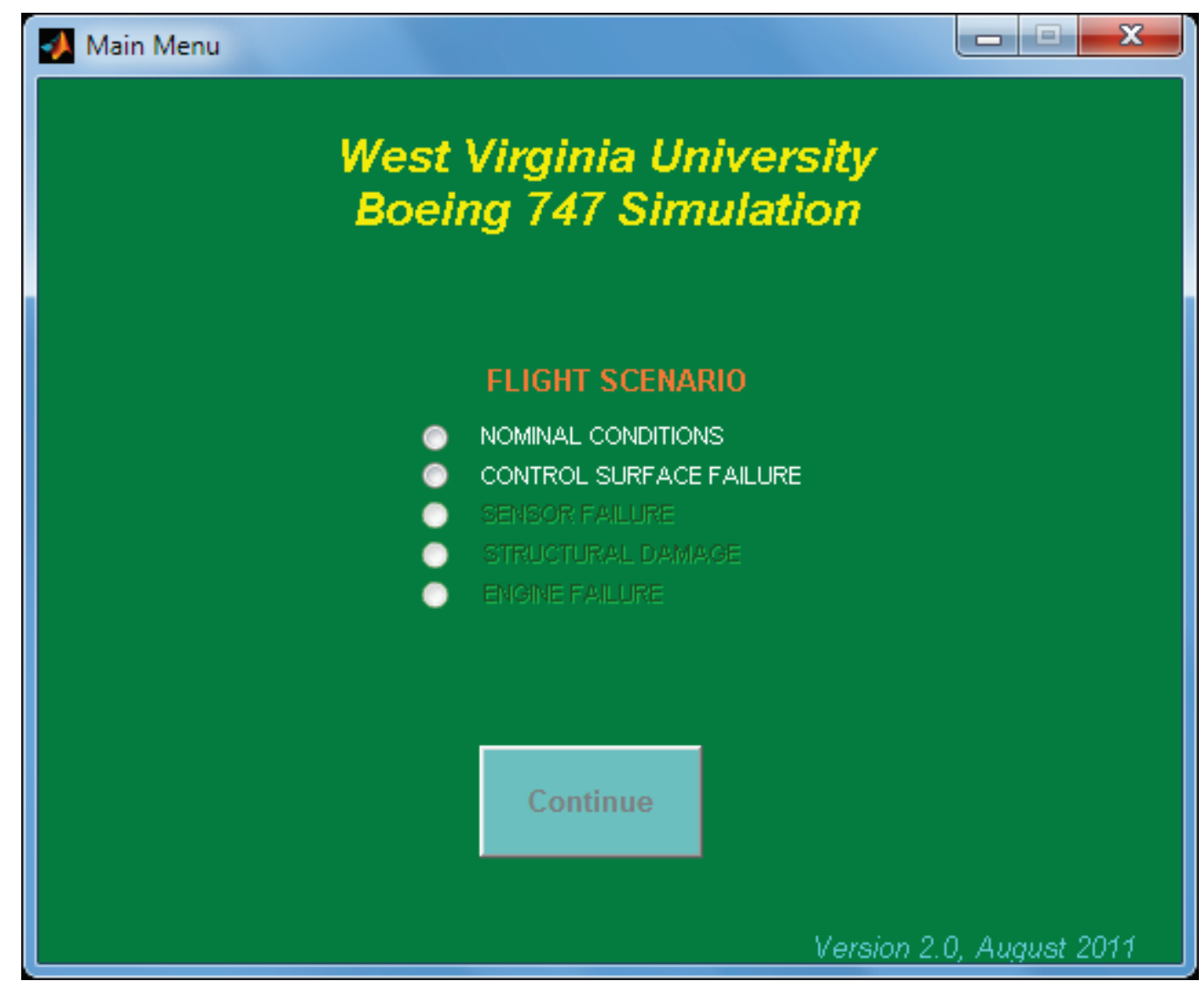

Figure B-0-3: Boeing 747 Simulation Main Menu 
Select the desired experiment from the Flight Scenario options and click continue. The next menu to appear will be the Pilot Input Menu (Figure B-4). Select the All Joystick Generated option and click continue. After pressing continue, make sure that four files were copied by checking the command window in the main MATLAB interface (Figure B-5)

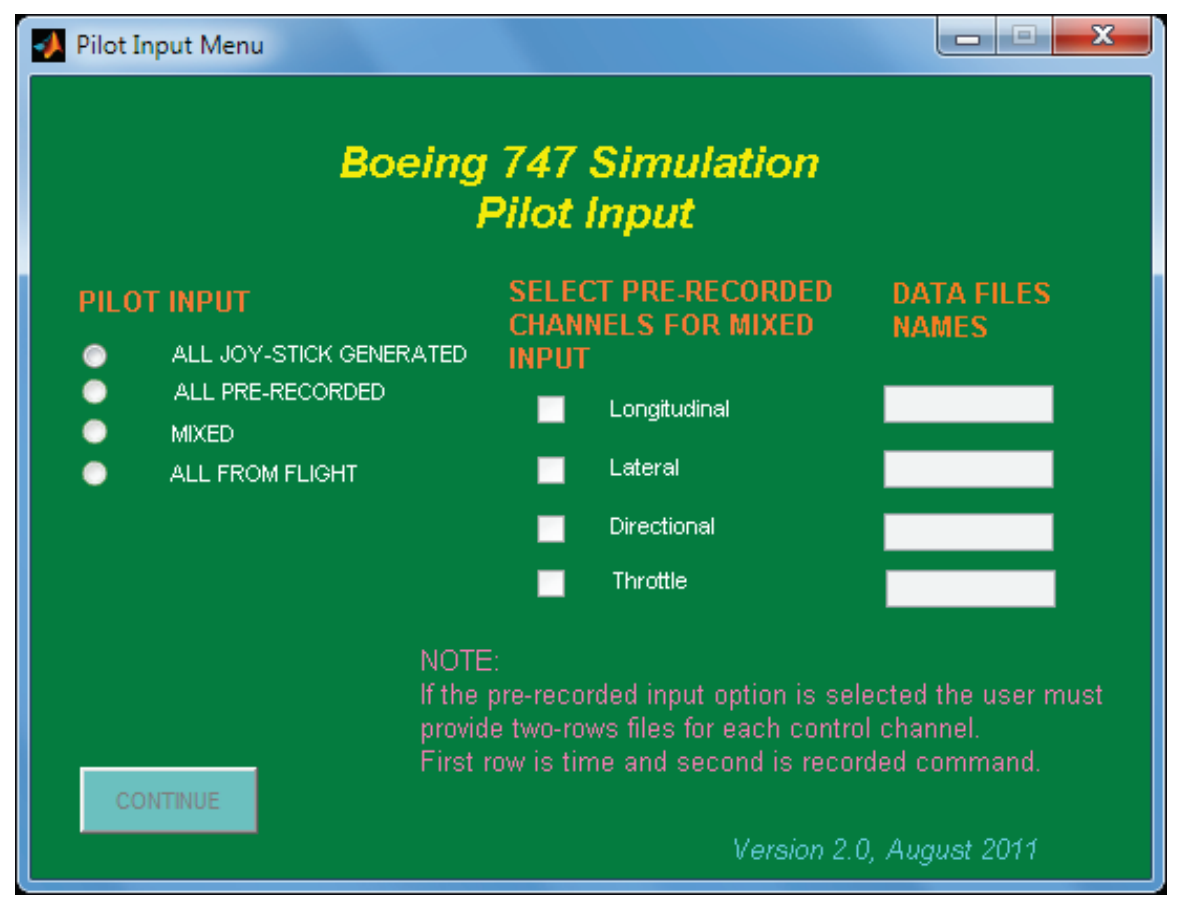

Figure B-0-4: Pilot Input Menu

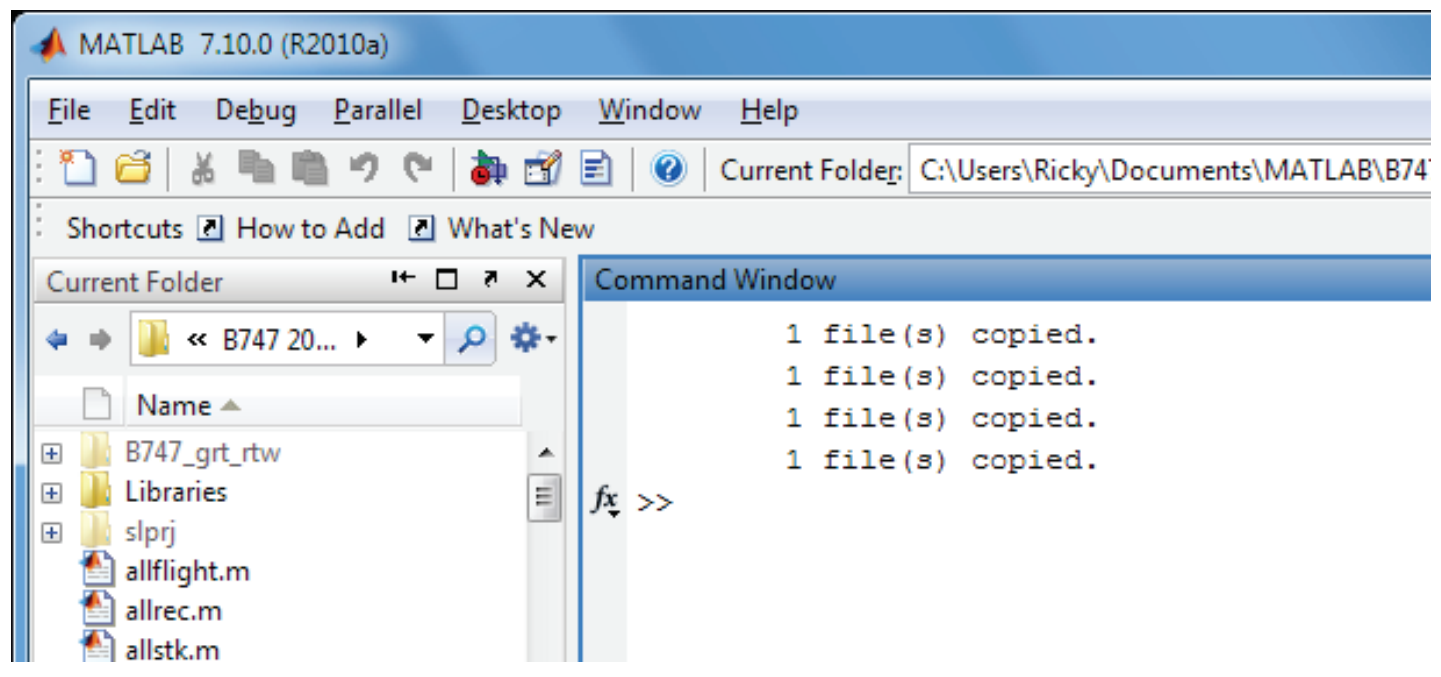

Figure B-0-5: Proper Loading of the Pilot Input Files 
Click continue on the next screen to select the given initial conditions setting (Figure B-6). Otherwise, select the proper initial condition and then press continue.

If a failure was selected from the initial main menu, the Failure Conditions Menu will open (Figure B-7). From here, select the Failure Scenario, the Failed Control Surface, and enter in additional relevant information in the boxes before pressing the continue button.

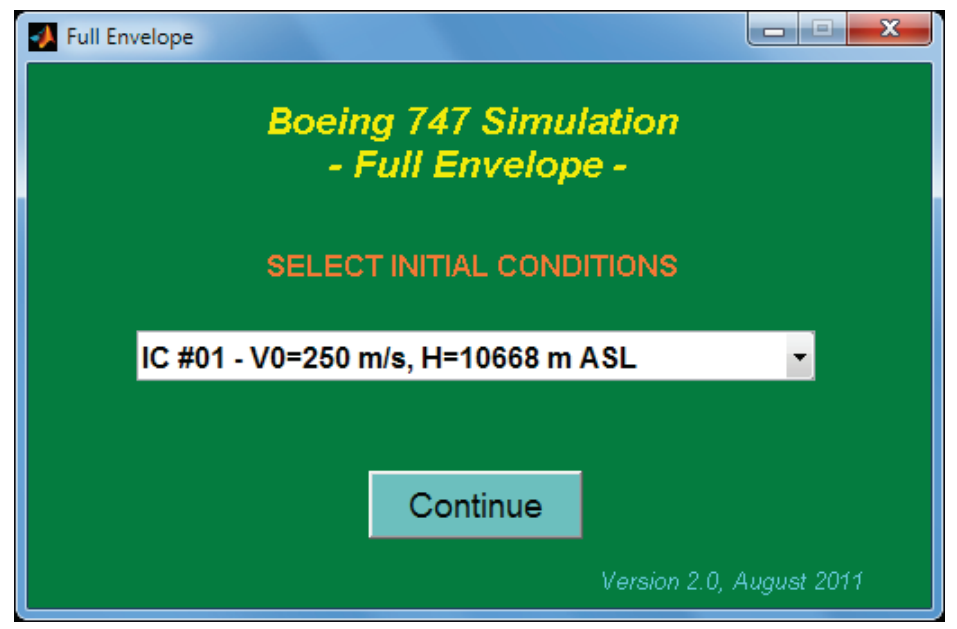

Figure B-0-6: Initial Conditions Selection

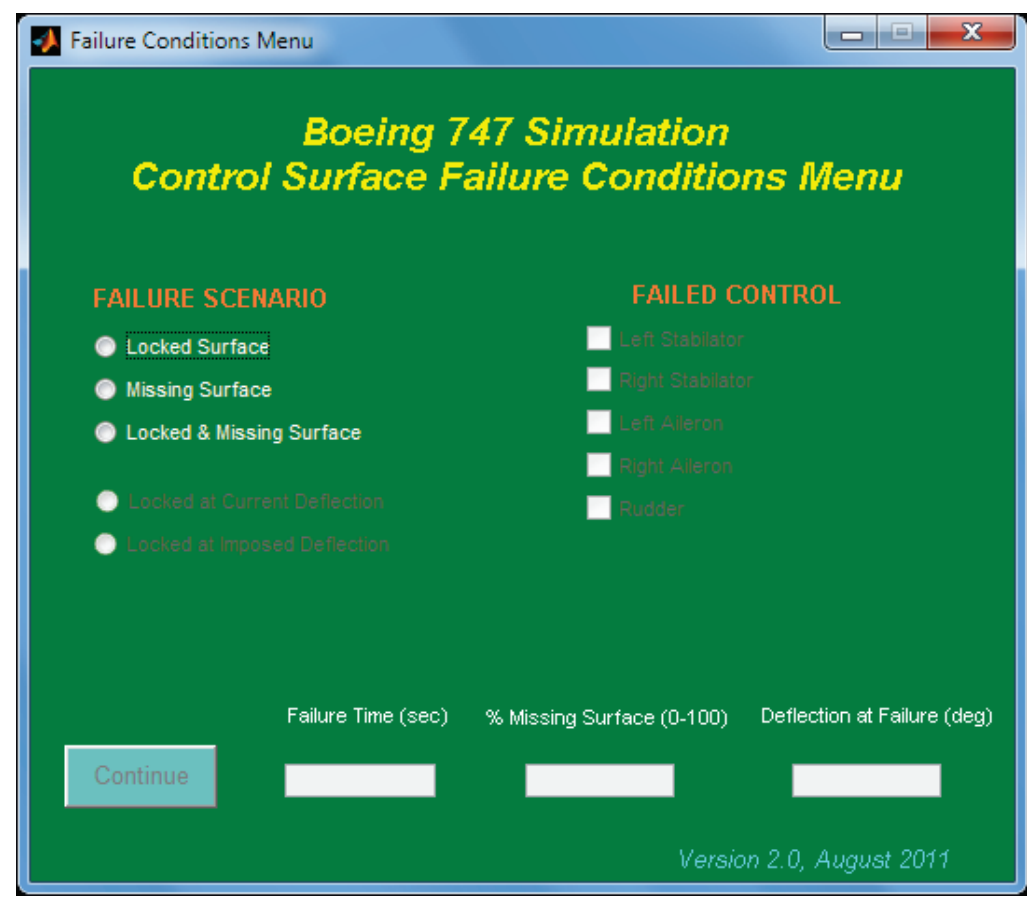

Figure B-0-7: Control Surface Failure Conditions Menu 
Whether a failure was selected in the opening menu or nominal conditions was selected, the following menu allows for the placement of the engines on the aircraft (Figure B-8). Select the number of engines from the top radio-buttons and then enter the location, in meters, from the aircraft's center of gravity. Suggested engine locations are shown in the graphic. When all engine locations are entered, press the continue button.

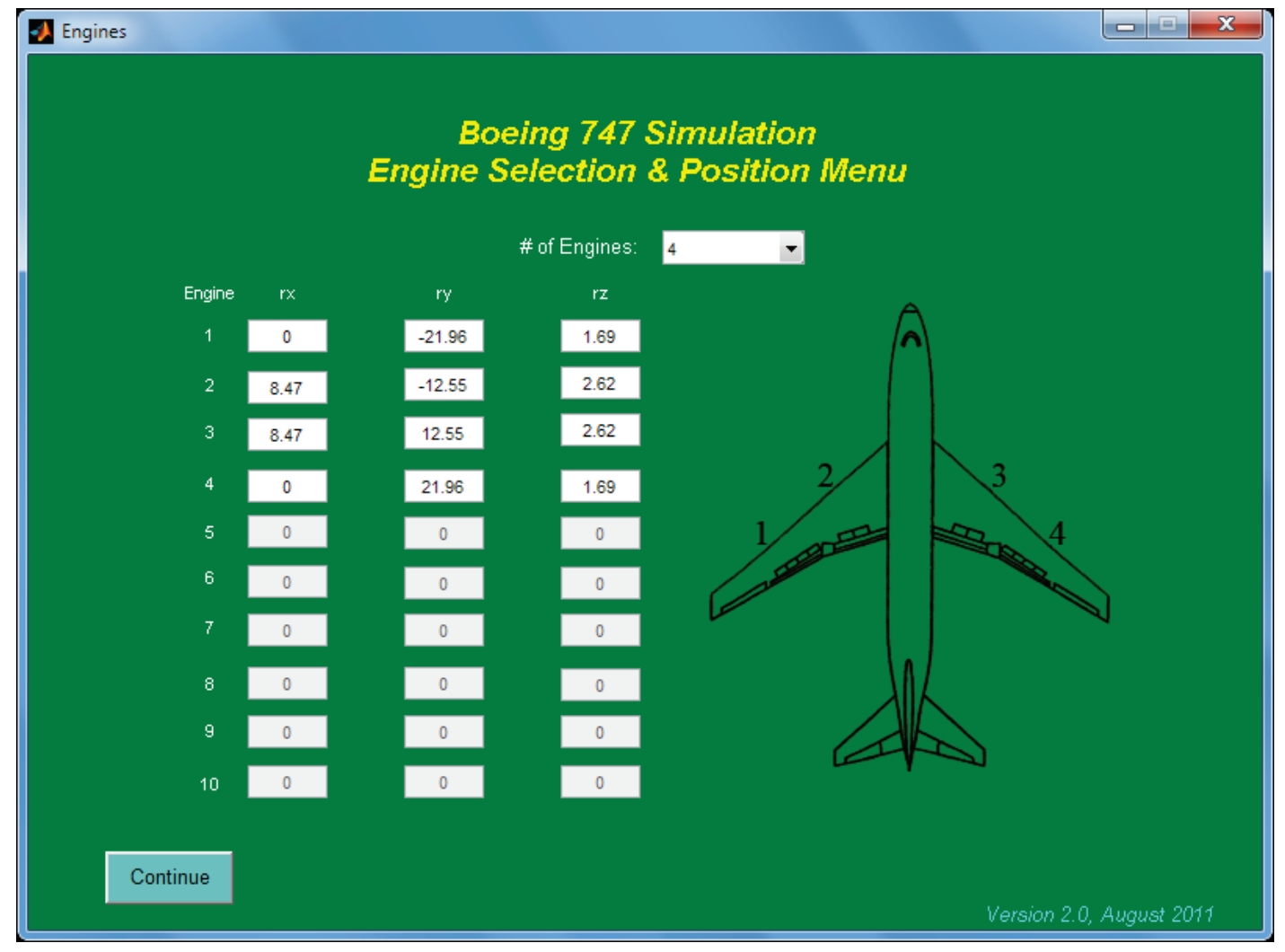

Figure B-0-8: Engine Selection and Position Menu

The WVU B747 Simulink model will load (Figure B-9). 


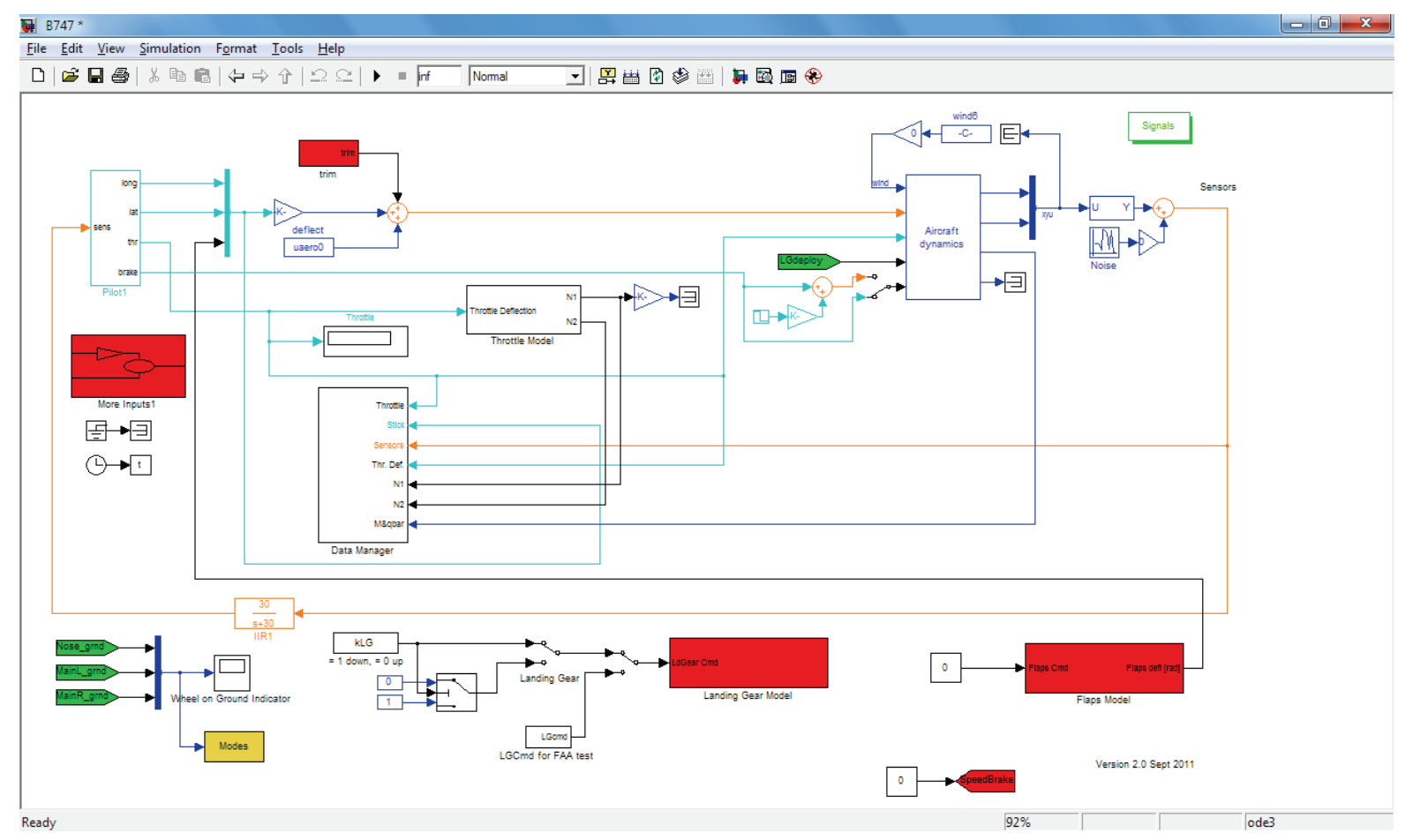

Figure B-0-9: WVU Boeing 747 Simulink Model

From the Scopes menu (Figure B-10), select the parameters that will need to be monitored during the flight and press the ok button. Additionally, if flight visualization is required, press the Open FlightGear button. The FlightGear initialization window will open (Figure B-11). When the program is finished loading, press the $\mathrm{V}$ button on the keyboard to scroll through available views until either the model view or helicopter view is selected (Figure B-12). When ready, press the Start Simulation button from within Simulink (Figure B-13). 


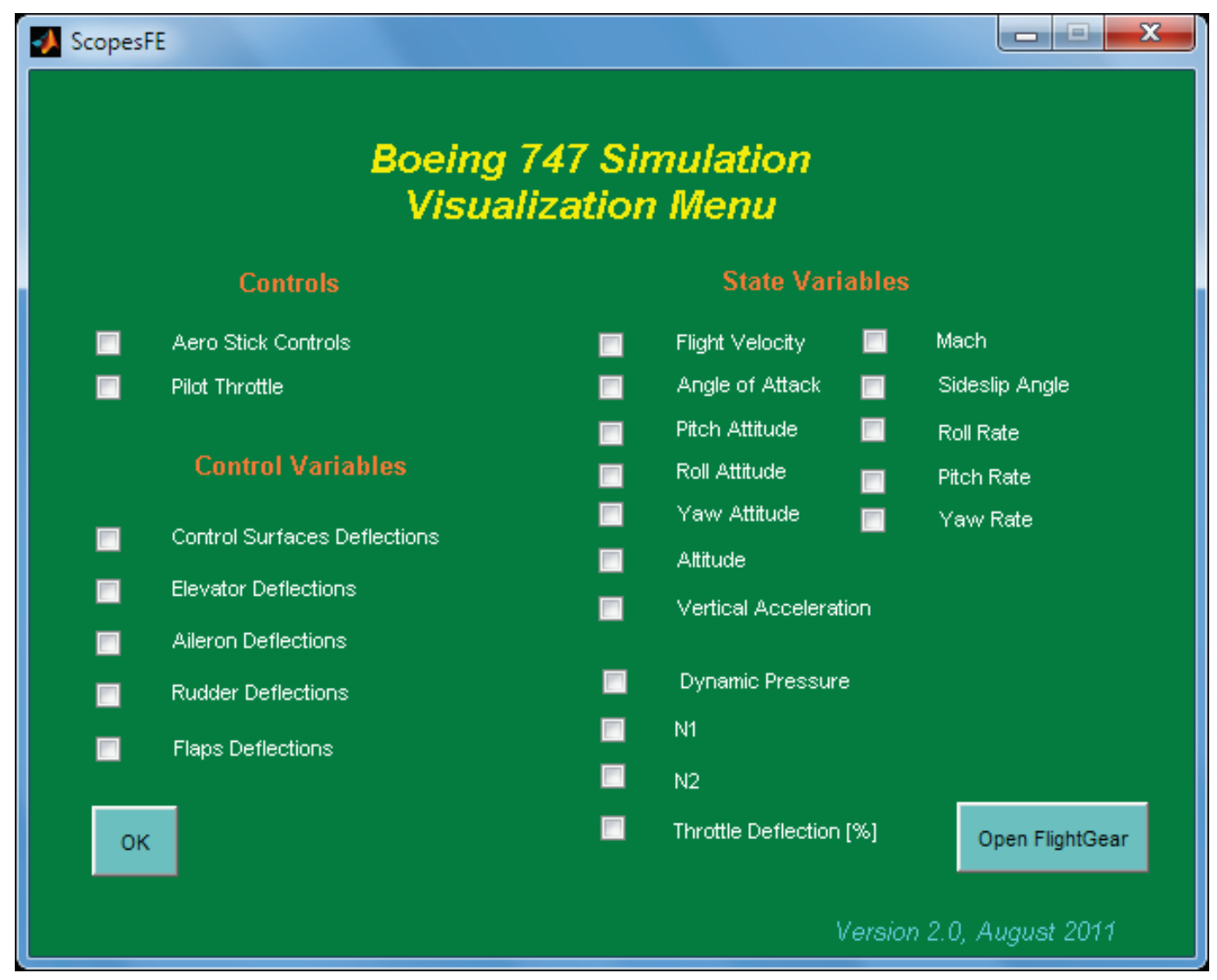

Figure B-0-10: Flight Visualization Menu

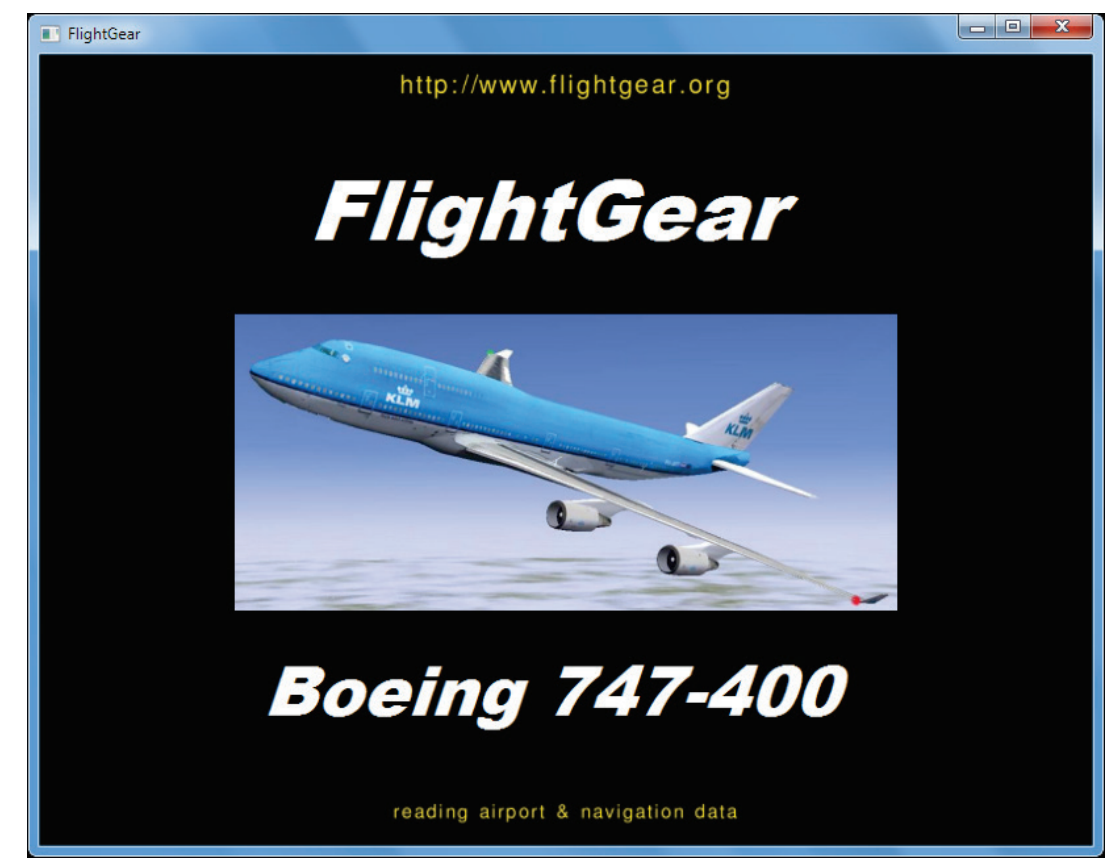

Figure B-0-11: FlightGear Initialization

B-7 


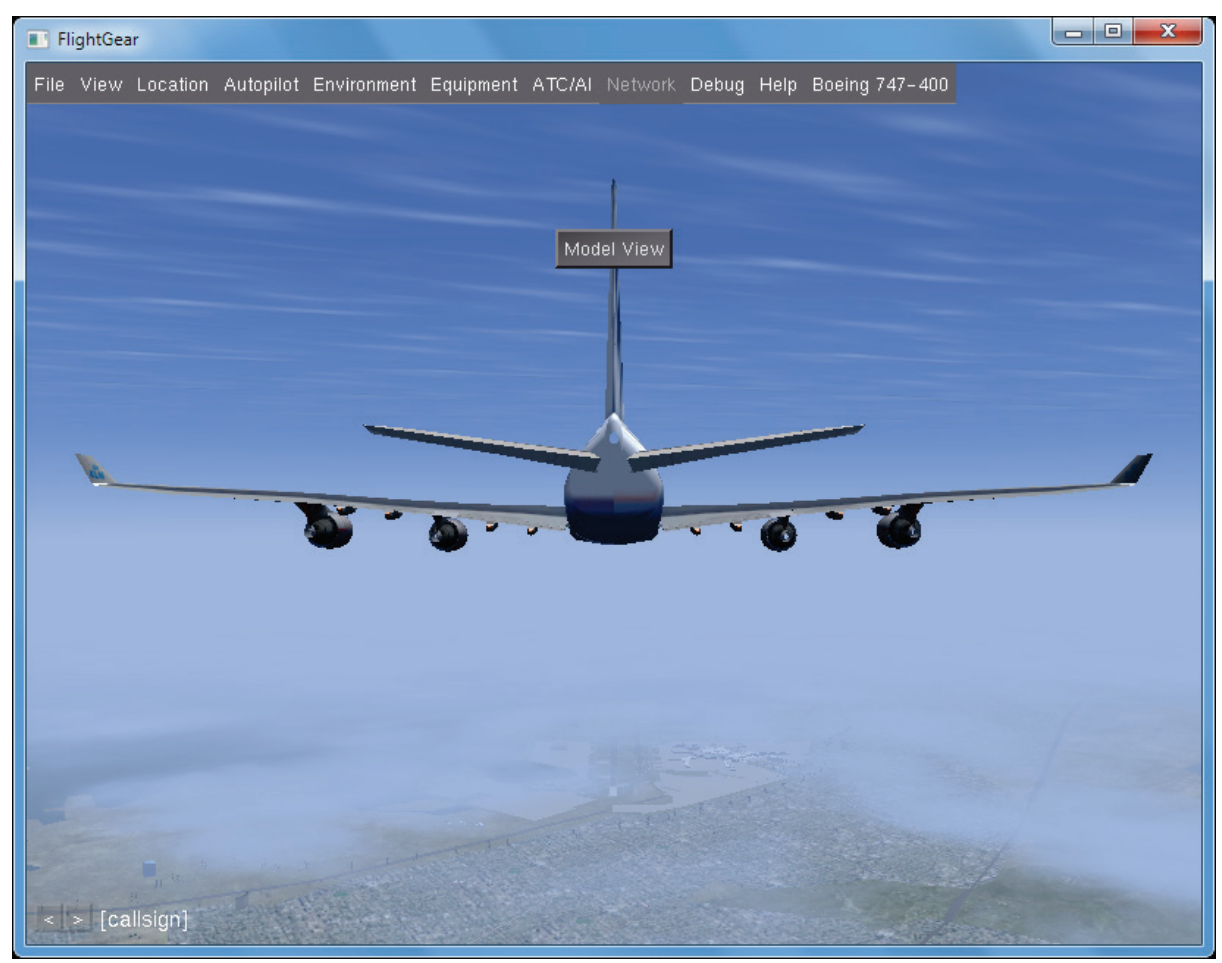

Figure B-0-12: FlightGear Window with Operating Flight Visualization

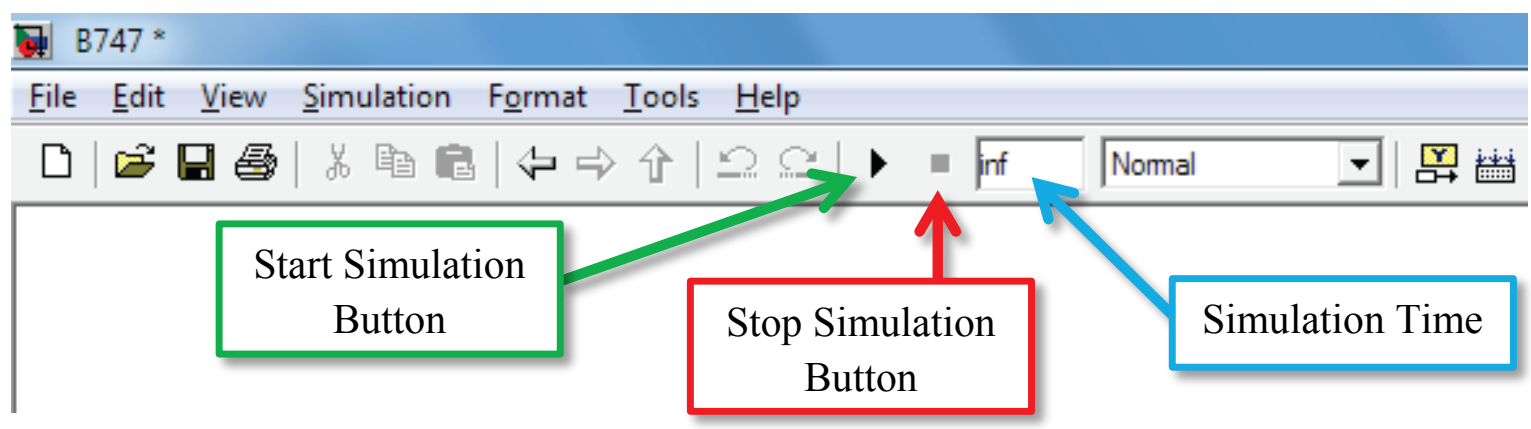

Figure B-0-13: Simulink Start and Stop Simulation Button and Simulation Time Highlighted 